Author-produced version of International Journal of Geographical Information Science, vol. 23 (7), pp. 877-921 Published: 2009 doi : $10.1080 / 13658810802022814$

Original paper available at Taylor \& Francis http://www.tandf.co.uk

\title{
Qualified Topological Relations between Spatial Objects with Possibly Vague Shape
}

BEJAOUI Lotfi, PINET François, BEDARD Yvan, SCHNEIDER Michel 
Broad boundaries are generally used to represent objects with fuzzy spatial extents. This concept is typically defined as a polygonal zone that should respect both connectedness and closeness conditions. Therefore, some real configurations, like regions with a partially broad boundary (e.g., a lake with rocker and swamp banks), are considered invalid. The main objective of this paper is to represent different levels of spatial fuzziness and consider these levels during the identification of topological relations. Then, we define a fuzzy spatial object as a minimal extent and a maximal extent. Topological relations are identified by a 4Intersection matrix that describes four subrelations between the minimal and the maximal extents. For fuzzy regions, 242 relations are distinguished and classified into 40 clusters. This approach permits the representation of partially fuzzy objects as well as the expression of integrity constraints and spatial queries with different levels of fuzziness.

Keywords: Fuzzy spatial objects; topological relations; partial fuzziness; spatial query; integrity constraints; integration

\section{Introduction}

To satisfy the requirements of several categories of users, Geographic Information Systems (GIS) and spatial databases provide tools to store, retrieve, analyze, and display spatial data. Ensuring their usability requires controlling the spatial data quality, which can be degraded by several types of imperfections. Several approaches (Smithson 1989, Fisher 1999, Mowrer 1999, Duckham et al. 2001) proposed different categorizations of data imperfections that are generally caused by the complexity of reality and limitations of instruments and processes used in the measurements (Bédard 1987). Moreover, inappropriate spatial data representations can also be another source of data quality degradation (Yazici et al. 2001, Shu et al. 2003, Dilo 2006). Spatial reality is generally forced to be represented by crisp spatial object types (i.e., points, lines, and regions), whereas many spatial objects are inherently fuzzy (e.g., forest stand, pollution zone, valley, or lake). Fuzziness occurs when it is difficult to distinguish an object's boundaries geometry components from other spatial objects. Using crisp spatial object types to represent fuzzy spatial objects entails a clear gap between the spatial reality and its formal representation in databases and GIS (Cheng et al. 2001, Yazici et al. 2001).

Pertinent solutions were found to overcome the "classical" sources of spatial data quality degradation (Bédard 1987, Goodchild 1995, Guptill and Morisson 1995, Ubeda and Egenhofer 1997, Frank 2001, Van Oort 2006, Devillers et al. 2007, Duboisset et al. 2007). Several approaches (Burrough and Frank 1996, Cohn and Gotts 1996, Clementini and Di Felice 1997, Erwig and Schneider 1997, Schneider 2001, Xinming 2004, Pfoser et al. 2005, Dilo 2006) have studied specificities of fuzzy spatial objects to determine their appropriate representation. A review of the literature in this domain stresses that current GIS and spatial database systems do not offer the specific structure to formally represent this type of object (as pointed by Clementini and Di Felice 1997 ten years ago). With regard to this problem, researchers are increasingly more motivated to model spatial fuzziness in order to: (1) reduce the gap between the geographic reality and the spatial models (Cohn and Gotts 1996), (2) provide formal modeling tools to represent spatial fuzziness (Yazici et al. 2001), and (3) specify spatial queries involving fuzzy spatial objects (Erwig and Schneider 1997). In the same way, the spatial data integration requires the extraction of heterogeneous representations of the same objects from different data sources. The main difficulty lies in choosing one of them when no information exists about their quality (Rodriguez 2005). By using a spatial 
model that supports spatial fuzziness, it becomes possible to merge different representations in such a way that the integration result looks like a fuzzy spatial object. For example, figure 1 shows a spatial object that has the representation $A$ in a first source and the representation $B$ in a second one. The integration result can correspond to one fuzzy geometry made up of $A$ and $B$ (figure 1). The intersection of $A$ and $B$ corresponds to the certain part (i.e., the part that exists in both representation $A$ and representation $B$ ) or the minimal extent of the spatial object. However, the union is the maximal extent that the object can fill; it groups the certain and the uncertain parts (i.e., a geometry part is uncertain when it does not exist in all candidate representations for the integration) of the geometry. Indeed, there are strong and different motivations to present pertinent solutions in order to adequately model the spatial fuzziness.

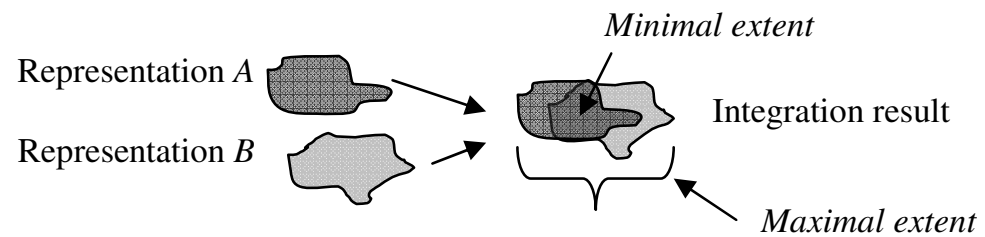

Figure 1. Integration of different spatial representations of a same object (e.g., lake)

To model fuzzy spatial objects, researchers were firstly inspired by the modeling of crisp spatial objects. In general point-set topology (Egenhofer and Herring 1990), crisp spatial objects are typically decomposed into three mutually disjoint topological invariants: an interior, a boundary, and an exterior. Several approaches (Clementini and Di Felice 1997, Xinming 2004, Reis et al. 2006) extend the crisp models by identifying other topological invariants for the fuzzy spatial objects. For example, Clementini and Di Felice (Clementini and Di Felice 1997) distinguish three topological invariants for fuzzy regions: an interior, a broad boundary (i.e., a two -dimensional boundary), and an exterior. In this approach, the fuzziness is correlated to the broad boundary, which should respect the closeness and the connectedness conditions (Clementini and Di Felice 1997, Xinming 2004). Thus, any representation that does not verify these conditions is considered invalid. Nonetheless, the fuzziness can also characterize only some parts of an object's geometry. For example, figure 2 shows a lake surrounded by rocker and swamp banks at the same time (figure 2). We denote this kind of feature as partially fuzzy spatial objects that cannot be represented by existing models. Then, the main questions are: How is it possible to define an exact model where different levels of fuzziness could be considered? How can we retain this expressivity during the specification of topological relations between such objects?

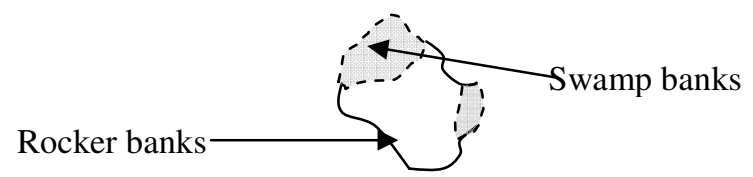

Figure 2. A lake with partially fuzzy boundaries

The first objective of this paper is to allow the representation of three levels of fuzziness: crispness, partial fuzziness, and complete fuzziness. Modeling fuzzy spatial objects requires a framework for identifying topological relations. The second objective is to consider the different levels of spatial fuzziness in the identification of topological relations between fuzzy spatial objects. In several studies (Clementini and Di Felice 1997, Xinming 2004, Reis et al. 2006), topological relations can be identified by enumerating the intersections between the topological invariants of the fuzzy objects involved. For each model, the number of relations depends upon the number of topological invariants. In this work, we look for an expressive model in which it is possible to specify the fuzziness level of the topological relation 
instances. We think that it would be pertinent for the user to know whether objects are weakly or strongly disjoint. Accordingly, the third specific objective of this work is to classify the topological relations according to their fuzziness level.

The remainder of the paper is organized as follows. In section 2, we present previous work on the modeling of fuzzy spatial objects and their topological relations. In section 3, we present three fuzzy spatial object types: fuzzy point, fuzzy line, and fuzzy region. Then, section 4 gives a proposition based on the 9-Intersection model (Egenhofer and Herring 1990) in order to identify the topological relations among fuzzy spatial objects. The model is applied to fuzzy regions, and their topological relations are studied in detail in the appendix. As a result of this approach, 242 relations can be distinguished through a 4-Intersection matrix. Section 5 proposes a hierarchical clustering of topological relations between fuzzy regions, and section 6 explains how to use our approach to express spatial queries and integrity constraints. In section 7, our model is compared with existing approaches (Cohn and Gotts 1996, Clementini and Di Felice 1997, Xinming 2004). Finally, section 8 presents our conclusions and discusses future research.

\section{Previous work}

\subsection{Spatial vagueness}

According to (Erwig and Schneider 1997, Hazarika and Gotts 2001, Pfoser et al. 2005), spatial vagueness can characterize the position or shape of an object's spatial extent. From this perspective, the shape fuzziness refers to the difficulty of distinguishing one object's shape from other objects' shapes. Fuzziness is an intrinsic property of an object that certainly has an extent in a known position but cannot or does not have a well-defined shape (Erwig and Schneider 1997). For instance, a region can be fuzzy when it is surrounded by broad instead sharp boundaries. Spatial vagueness can also characterize well-defined (or crisp) objects when there is uncertainty about object positions despite their sharp shapes; we refer to this scenario as positional vagueness. Figure 3 shows this categorization of spatial vagueness into "shape fuzziness" and "positional vagueness". In this paper, we only deal with the formal representation of fuzzy spatial objects (i.e., objects that have fuzzy shapes) and the topological relations between them.

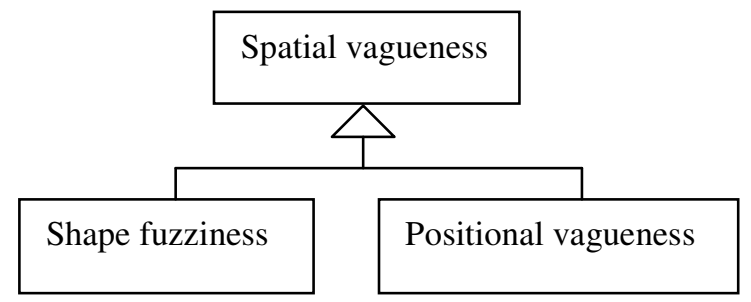

Figure 3. Categorization of spatial vagueness

In general, we distinguish between at least two categories of models used to represent spatial vagueness. In the first category, crisp spatial concepts are transferred and extended to formally express spatial vagueness; we speak about exact models as explained in the next section (Cohn and Gotts 1996, Clementini and Di Felice 1997, Erwig and Schneider 1997). In the second category, three principal mathematical theories are generally used: (1) models based on the fuzzy logic (Zadeh 1965) (e.g., Altman 1987, Burrough 1989, Brown 1998, Schneider 2001, Xinming 2004, Hwang and Thill 2005, Dilo 2006), which can be used to represent continuous phenomena such as temperature, (2) models based on rough sets (e.g., Ahlqvist et al. 1998, Worboys 1998), which represent the fuzzy spatial objects as a pair of 
approximations (upper and lower approximations), and (3) models based on probability theory (e.g., Burrough and Frank 1996, Pfoser et al. 2005), which is principally used to model errors of position and attributes. In the next section, we present works that formally define fuzzy spatial objects.

\subsection{Formal definitions of fuzzy spatial objects}

2.2.1 Definitions based on exact models. The Egg-Yolk theory (Cohn and Gotts 1996) is an extension of the RCC (Region Connection Calculus) model (Randell and Cohn 1989, Cohn et al. 1997), it introduces the concept of regions with large boundaries. In fact, a fuzzy region is composed of two crisp regions. The inner region called «yolk» (i.e., the certain part of the geometry), and it is surrounded by an outer region called "white" (i.e., the large boundary or the uncertain part of the geometry). The union of the "yolk" and the "white" corresponds to the "egg". Because points and lines are ignored in RCC model, their fuzziness is not studied in this approach. In addition, fuzzy regions with empty "yolk" or empty "egg" are not admitted. Thus, the crisp regions cannot be represented through the Egg-Yolk theory.

In the same way, Clementini and Di Felice (Clementini and Di Felice 1997) define the regions with broad boundaries based on the general point-set topology (Egenhofer and Herring 1990). Thus, a region with a broad boundary $A$ is made up of two simple crisp regions $A_{1}$ and $A_{2}$, where $A_{1} \subseteq A_{2}$. The broad boundary represents the fuzziness, and it is equal to the closure of their difference $\Delta A=A_{1}-A_{2}$. In this approach, $A_{1}$ and $A_{2}$ should be topologically consistent; this means that they should be bounded, regular, and closed sets in $\mathfrak{R}^{2}$ (Clementini and Di Felice 1997). Moreover, the authors distinguish two kinds of fuzzy lines: broad lines (i.e., all of the line is large) and lines with broad boundaries (i.e., the line's endpoints are ill-defined). Xinming (Xinming 2004) extends this approach and proposes a more detailed formal definition of fuzzy regions, where he distinguishes four mutually disjoint components: the interior, the boundary's interior, the boundary's boundary, and the exterior (figure 4).

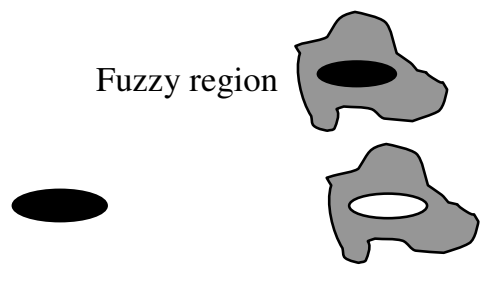

(a) Interior

(b) Boundary's boundary

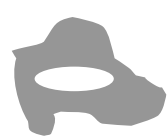

(c) Boundary's Interior

Figure 4. Topological invariants of a simple fuzzy region in (Xinming 2004)

The condition $A_{1} \subseteq A_{2}$ in (Clementini and Di Felice 1997) does not exist in (Erwig and Schneider 1997). In fact, Erwig and Schneider (Erwig and Schneider 1997) are interested in another kind of vagueness, where a vague region is a composed geometry. The geometry's components belong to a pair of subsets. First, the kernel subset contains the subregions definitely belonging to the vague region. Second, the boundary subset contains the subregions possibly belonging to the vague region. Likewise, the vague points and the vague lines are respectively defined as a pair of subsets of points and lines. Crisp spatial objects can be expressed through this model when the boundary subset is empty. Figure 5 gives an example of a vague region $\mathrm{A}$, in which the white subregions compose to the boundary subset and the gray ones compose the kernel. 


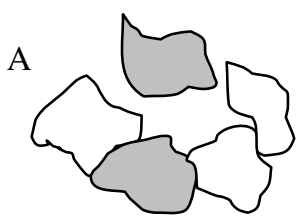

Figure 5. Vague region in (Erwig and Schneider 1997)

2.2.2 Models based on mathematical approaches. Roy and Stell (Roy and Stell 2001) deal with indeterminacy, which is defined as knowledge vagueness. They define an indeterminate region through rough sets (Pawlak 1994). An indeterminate region is composed of a lower and an upper approximation. The difference between these approximations represents the fuzzy part of the region. When this difference is empty, the region is crisp because the two approximations are equal (Roy and Stell 2001).

Fuzzy sets theory is also used to represent fuzzy spatial objects (Robinson and Thongs 1986, Altman 1987, Burrough 1989, Zhan 1997, Schneider 2001, Yongming and Sanjiang 2004, Dilo 2006). Zhan (Zhan 1997) and Dilo (Dilo 2006) interpret a fuzzy spatial object as a fuzzy subset. In (Zhan 1997), the membership function of the fuzzy spatial object is decomposed to $n \alpha$-cuts (an $\alpha$-cut is the set of points with a membership value $\alpha$ or higher (e.g. Godjjevac 1999)) in order to facilitate its interpretation. Xinming (Xinming 2004) defines the fuzzy spatial objects in two different ways. The first definition should respect the properties of the crisp topological space. The second definition should respect topological properties in a fuzzy topological space. In this context, the concept of fuzzy topology is a generalization of crisp topology, in which the sets belonging to the universe are fuzzy (i.e., the membership value is $\alpha$, where $0 \leq \alpha \leq 1$ ). Schneider (Schneider 2001) presents formal definitions of fuzzy spatial object types through fuzzy subsets. For example, a fuzzy region is defined as a generalization of a crisp region in which an arbitrary point of space has a partial membership to the region (Schneider 2001).

In the next section, we highlight some approaches that study the specificities of the topological relations between fuzzy spatial objects.

\subsection{Topological relations between fuzzy spatial objects}

Topological relations are very important in GIS, especially in the specification of integrity constraints (Ubeda and Egenhofer 1997) and spatial queries. These relations are based only on the shape of objects and are totally independent of both the coordinates system and geometric transformations. The use of special shapes to express shape fuzziness should imply a different approach for identifying their topological relations. In the crisp context, several models (Egenhofer and Herring 1990, Egenhofer and Franzosa 1991, Mark and Egenhofer 1994, Cohn et al. 1997) studied the specification of topological relations in GIS and spatial databases. These models are based on two main approaches: general point-set topology (Egenhofer and Herring 1990) and mereology ${ }^{1}$ (Varzi 2004). First, the 9-interesections model (Egenhofer and Herring 1990) is an extension of the 4-Intersection model (Egenhofer 1989). Based on point-set topology, the 9-Intersection model distinguishes 8 relations between two simple regions (Disjoint, Meet, Overlap, Contains, Inside, Equal, Covers, Covered by), 36 between two simple lines, 19 between a simple region and a simple line, 2 between two points, 3 between a point and a simple line, and 3 between a point and a simple region. In the context of mereology, a first model called RCC-5 (Randell and Cohn 1989) distinguished 5

\footnotetext{
${ }^{1}$ The region is defined as the elementary component of the space, i.e. points and lines are not considered
} 
topological relations among two simple crisp regions. This model was then extended to distinguish 8 topological relations, and it became known as RCC-8 (Cohn et al. 1997).

In the fuzzy context, topological relations can be specified by extending the RCC and 9Intersection models (Cohn and Gotts 1996, Clementini and Di Felice 1997, Erwig and Schneider 1997, Roy and Stell 2001, Xinming 2004). Erwig and Schneider (Erwig and Schneider 1997) use a three-valued logic to underline the spatial fuzziness. Then, an intersection between two topological invariants can be true, false, or maybe (i.e., when the boundary subset participates in the relation). In (Cohn and Gotts 1996), a topological relation between two Egg-Yolk regions $A$ and $B$ is specified through a $2 * 2$-matrix that enumerates four subrelations: $\operatorname{Egg}(A)-\operatorname{Egg}(B), \operatorname{Egg}(A)$ - Yolk(B), $\operatorname{Yolk}(B)-\operatorname{Egg}(A)$, and $\operatorname{Yolk}(A)-\operatorname{Yolk}(B)$ (figure 4). These four subrelations are those defined in the RCC-5 model: Partially Overlapping (PO), Proper Part (PP), Equal (E), Proper Part Inverse (PPI), and Distinct (D). With this approach, only 46 combinations correspond to the 46 possible topological relations drawn in (Cohn and Gotts 1996). Figure 6 presents the relation number 15 in (Cohn and Gotts 1996). The main advantage of this approach is its simplicity in identifying the topological relations. However, point, lines, and crisp regions are not covered.

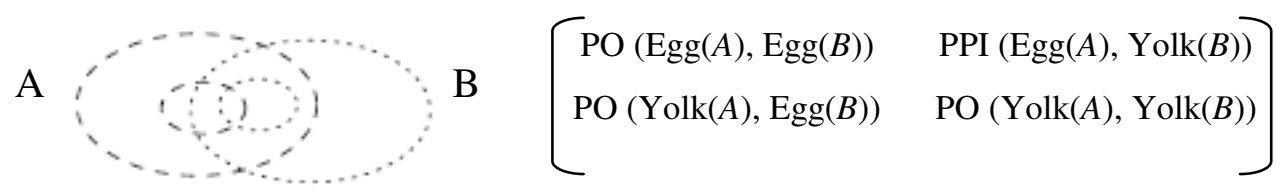

Figure 6. Identification of topological relations in (Cohn and Gotts 1996)

Clementini and Di Felice (Clementini and Di Felice 1997) studied approximate topological relations, which correspond to the topological relations between regions with broad boundaries. In fact, they use a $3 * 3$-matrix, in which the sharp boundary is replaced by a broad boundary. After considering a set of consistency rules for the matrix (i.e., twelve rules to eliminate a matrix that cannot be drawn) (Clementini and Di Felice 1997), only 44 relations can be distinguished and drawn among two simple fuzzy regions. These relations can then be grouped into 17 clusters, for which a conceptual-neighborhood graph was drawn. This approach can be very interesting when it is necessary to coarsely identify the topological relations between fuzzy regions. However, the model is not sufficient when the needs are more specific and the user has a clear idea regarding the relation between fuzzy regions. For example, figure 7 shows an example of two different relations absorbed by the same cluster and identified through the same matrix.

(1)

(2)

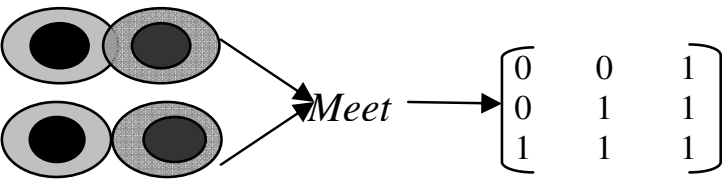

Figure 7. Identification of topological relations in (Clementini and Di Felice 1997)

In the same way, Reis et al. (Reis et al. 2006) use Clementini's model (Clementini and Di Felice 1997) in order to identify the topological relations between fuzzy lines. After considering the same 12 conditions defined in (Clementini and Di Felice 1997), 5 topological relations are identified between two broad lines and 77 between two lines with broad boundaries.

Xinming (Xinming 2004) presents an extension of the 9-Intersection model that identifies more topological relations than that of (Clementini and Di Felice 1997). He identifies 
topological relations through a $4 * 4$-matrix. Indeed, he distinguishes 152 topological relations and presents their correspondent matrices (see the example in the figure 8). The absence of relation clustering is the main limitation of the model. The model becomes useless in practice, however, since it is very difficult to easily and intuitively distinguish all of these relations. Furthermore, Xinming (Xinming 2004) does not distinguish between the inner and outer boundaries. Hence, many relations cannot be specified through a $4 * 4$-matrix.

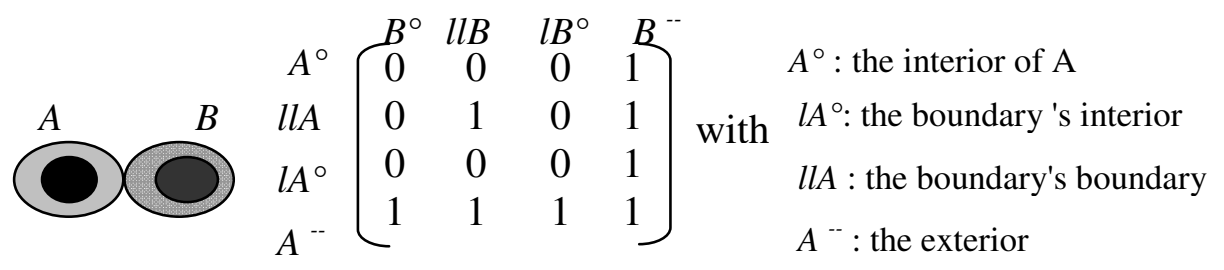

Figure 8. Identification of topological relations in (Xinming 2004)

Fuzzy sets' theory is also used to identify the topological relations between fuzzy spatial objects (Zhan 1997, Schneider 2001, Dilo 2006). A topological relation in (Zhan 1997) is called $R$ (i.e., a parameter used to replace the eight relations of the 4-Intersection model (Egenhofer 1989)). For each pair of $\alpha$-cuts of considered regions, a subrelation $r$ is identified. Then, the possibility of the global relation $R$ is deduced from the number of subrelations $r$ that arise between the different $\alpha$-cuts. This approach is easy to use in practice, but it presents some complexity when the $\alpha$-cuts are non-uniformly distributed between 0 and 1. In the same way, Dilo (Dilo 2006) identifies six possible topological relations (i.e. Disjoint, Touches, Crosses, Overlaps, Within, and Equal) between two vague spatial objects. A topological relation is defined based on fuzzy operations (e.g., union, intersection, absolute difference, and bounded difference) applied to fuzzy subsets that represent vague spatial objects. According to Dilo (Dilo 2006), many topological relations may exist at the same time with different Truth degrees (e.g., Overlap $(A, B)$ with the Truth degree $=0,2 ;$ Meet $(A, B)$ with the Truth degree $=0,3 ; \operatorname{Disjoint}(A, B)$ with the Truth degree $=$ $0,5)$.

\subsection{Problem statement}

The exact models presented earlier (Cohn and Gotts 1996, Clementini and Di Felice 1997, Erwig and Schneider 1997, Xinming 2004, Reis et al. 2006) have the advantage of explicitly distinguishing objects' topological invariants. Through this discrete viewpoint of space, the specification of topological relations can be improved (Clementini and Di Felice 1997). For these reasons, we propose an exact model in order to achieve objectives. Nevertheless, we think that the existing models do not distinguish between different levels of fuzziness and are not sufficiently expressive to represent partial fuzziness. In reality, a fuzzy spatial region is not always surrounded by a large boundary everywhere. For example, a lake boundary can be broad in some locations and sharp in some others. This situation cannot be represented by existing exact models, because the connectedness condition is violated. The same problem is present for lines. Only two cases of fuzziness are distinguished in lines (cf. section 2.2.1), but a line can easily have a partially fuzzy interior. Moreover, the studied models are not sufficiently expressive in terms of topological relations since there is no distinction between the inner and outer boundaries for a fuzzy region. Some work tries to offer more expressivity by increasing the number of topological invariants (Xinming 2004). Nevertheless, the absence of relation clustering limits their practical use. Indeed, the main research questions of our paper are the following: 
1- How can we obtain more expressive definitions of the fuzzy spatial object types through an exact model? How can we represent partial fuzziness?

2- What are the topological relations between fuzzy spatial objects? How is it possible to identify topological relations between objects that have different levels of fuzziness? How can we formally identify these relations?

3- How can we classify the topological relations between fuzzy regions in order to facilitate their use in practice? How could resulting clusters reflect the fuzziness level of a topological relation?

\section{Fuzzy spatial objects}

In general, there is no agreement regarding the appropriate formal definition of fuzzy spatial objects, because fuzziness can be interpreted in different ways. It is not the objective of this work to unify these interpretations. We are interested in proposing an expressive and easy definition of fuzzy spatial objects through an exact model. In our approach, we transfer the Egg-Yolk's model into point-set topology context in order to both consider points and lines and permit the representation of partially fuzzy objects. From this perspective, we distinguish three fuzzy spatial object types: fuzzy points, fuzzy lines, and fuzzy regions. Each one of these is composed of $n$ crisp object types (i.e., point, line, and region) distributed into a pair of sets called (1) the minimal extent and (2) the maximal extent (figure 9). Figure 9 presents an example of fuzzy points, fuzzy lines, and fuzzy regions. A fuzzy point is a zone that we approximate to a crisp region containing all of positions that the point can possibly fill. The minimal extent of point is equal to its maximal extent because the fuzziness concerns a unique topological invariant: the interior (cf. section 3.1 for more details). For a fuzzy line (cf. section 3.2), the minimal extent is the union of the linear parts. However, its maximal extent can contain some fuzzy parts (i.e., presented as fuzzy points in figure 9 (b)) at which the line can have any shape. For a fuzzy region (cf. section 3.3), the fuzziness concerns the boundary. The minimal extent refers to the geometry when the boundary is as close as possible (i.e. it is drawn around the area which certainly belongs to the region). The maximal extent is the geometry of the object when the boundary is as far away as possible (i.e. it is drawn around the area which contains all of points possibly belonging to the region).

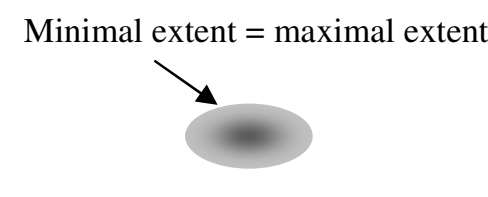

(a) Fuzzy point

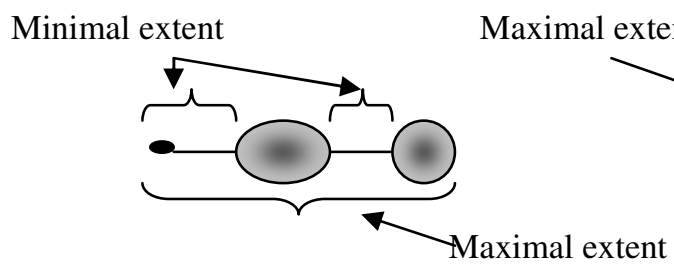

(b)Fuzzy line

(c) Fuzzy region

Figure 9. Minimal and maximal extents for (a) a fuzzy point, (b) a fuzzy line and (c) a fuzzy region

Generally, the minimal extent refers to the geometry's parts definitely belonging to the spatial object. The maximal extent corresponds to the object's geometry when fuzziness is taken into account and added to the minimal extent. Outside of the maximal extent, there are no spatial points that can possibly belong to the object. The number $n$ of crisp object types composing the fuzzy spatial object type is 1 for a fuzzy point (i.e., a zone that we represent as a crisp region composed of the quasi-totality of possible positions of the point (cf. section 3.1)), 2 for a fuzzy region (i.e., two crisp regions (cf. section 3.3)), and $n$ for fuzzy lines (i.e., 1 or $n$ points of the line are fuzzy (cf. section 3.4)). For example, a fuzzy region corresponds to a pair of crisp regions that respectively represent the minimal and maximal extents. This general definition of fuzzy spatial objects is based on the following principles: 
1- A fuzzy spatial object is a generalization of a crisp spatial object.

2- The minimal and the maximal extents are made up of crisp spatial object types. Only the combination of two extents corresponds to the fuzzy spatial object.

3- For the minimal and the maximal extents, the topological invariants should be mutually disjoint.

The first principle means that the spatial extent of a fuzzy object is crisp when its minimal extent is equal to its maximal one. The second principle requires that the minimal and maximal extents verify the topological consistency conditions of the crisp spatial object types (e.g., a crisp region should be connected). Finally, the third principle permits the identification of topological relations based on the intersections between the topological invariants of the minimal and maximal extents of two fuzzy spatial objects. In the next sections, we present our definitions of fuzzy point, fuzzy line, and fuzzy region.

\subsection{Fuzzy point}

In the crisp context, a point $p(x, y)$ is a 0 -dimensional object type that represents one position $(x, y)$ in space. The only topological invariant composing the shape of a crisp point is its interior. Because a point does not have a boundary (the boundary of an object with $\mathrm{N}$ dimensions has $\mathrm{N}-1$ dimensions), the fuzziness can characterize only the interior and thus the point itself. Semantically, a fuzzy point occurs when an intrinsic property of the point or a lack of knowledge does not permit certainty regarding its position. For such a case, the spatial extent of the object is typically replaced by a zone that we represent as a crisp region composed of the family of positions that the point can fill (figure 10). The closure ${ }^{2}$ of the crisp region represents an infinity of possible positions for the point. Consequently, a fuzzy point does not have a minimal extent; it only has a maximal extent.

\begin{tabular}{|c|c|}
\hline Simple Crisp point & Fuzzy point \\
\hline$\bullet$ & \\
& \\
\hline
\end{tabular}

Figure 10. Fuzzy point

Since a simple fuzzy point corresponds, in fact, to a simple crisp region, it should verify the following conditions:

1- The closure is a non-empty connected regular closed set.

2- The interior is a non-empty connected regular open set.

3- The boundary and exterior are connected.

To provide an example of a fuzzy point, consider an application to help the fire brigades in their interventions. Generally, a fireman cannot precisely localize the fire source. However, he can draw an area in which the fire source should exist. This intervention area corresponds to a fuzzy point and can be represented through our model. It is clear that the size of the region representing the fuzzy point depends on the fuzziness level (i.e., a larger region refers to a fuzzier point).

\subsection{Fuzzy line}

A simple crisp line is a one-dimensional object type made up of an interior limited by two endpoints. The boundary of a crisp line corresponds to its endpoints, whereas the interior is

\footnotetext{
${ }^{2}$ The closure, in point set topology, is the union of the interior and the boundary.
} 
the set of points connecting them. The fuzziness can characterize any point of the line. Consequently, the line boundary can be partially or completely fuzzy while the interior remains well-defined; we then speak about lines with fuzzy boundaries. In the same way, the interior can be partially or completely fuzzy while the endpoints are well-defined; we then speak about lines with partially and completely fuzzy interiors, respectively (figure 11). The extreme case of line fuzziness arises when all topological invariants of the line (i.e. the interior and the boundary) are fuzzy (figure 11). Thus, a completely fuzzy line corresponds to a crisp region that represents the set of positions that the line can fill. However, a completely crisp line is a particular case of fuzzy lines, for which all of the interior and both endpoints are well defined. The different cases of line fuzziness can be combined as presented in figure 11 . To underline the different levels of line fuzziness, we use four adverbs: (1) weakly, (2) fairly, (3) strongly, and (4) completely. The term "weakly" indicates that one of the topological invariants is partially fuzzy. The term "fairly" reflects either complete fuzziness of one of topological invariants or the case where the interior and endpoints are partially fuzzy at the same time. The term "strongly" specifies complete fuzziness for one of topological invariants and partial fuzziness for the second one. Finally, the term "completely" is used to express total fuzziness of the line's components. Figure 11 shows a symmetrical matrix, in which fuzziness increases from "none" in the upper-left cell to "completely" in the lower-right cell through a progression including "weakly", "fairly," and "strongly".

\begin{tabular}{|l|c|c|c|}
\hline Crisp boundary & Crisp interior & Partially fuzzy interior & Completely fuzzy interior \\
\hline $\begin{array}{l}\text { Partially fuzzy } \\
\text { boundary }\end{array}$ & $\begin{array}{l}\text { nome } \\
\text { fuzzy boundary }\end{array}$
\end{tabular}

Figure 11. Fuzzy lines

In our model, a fuzzy line is typically composed of two-dimensional parts that correspond to the line's fuzzy points and one-dimensional parts that represent the minimal extent of the line. Thus, the maximal extent is the union of the one-dimensional and two-dimensional parts. The interior of maximal extent corresponds to the union of the interiors of the one-dimensional and two-dimensional parts. In the same way, the boundary of the maximal extent is the union of the boundary of the one-dimensional and two-dimensional parts. Figure 12 presents the different cases of fuzzy line decomposition. We should note that the different representations of a line, in figure 12, indicate a set of pictograms that we use to show different types of fuzziness for lines. In other terms, these representations are not based on a mathematical model that allows to consider error component of spatial data as in (Chrisman 1991).

\begin{tabular}{|c|c|c|c|c|c|}
\hline Fuzzy line & \multicolumn{2}{|c|}{ Extents } & \multicolumn{3}{|c|}{ Topological invariants } \\
\hline & \multirow{2}{*}{ Minimal extent } & \multirow{2}{*}{0} & Interior & & \\
\hline & & & Boundary & 0 & 0 \\
\hline & \multirow[t]{2}{*}{ Maximal extent } & \multirow[b]{2}{*}{$\bullet$} & Interior & & - \\
\hline & & & Boundary & 0 & 0 \\
\hline \multirow{2}{*}{$\cdots$} & \multirow[t]{2}{*}{ Minimal extent } & \multirow{2}{*}{0} & Interior & \multicolumn{2}{|c|}{$\longrightarrow$} \\
\hline & & & Boundary & - & \\
\hline
\end{tabular}




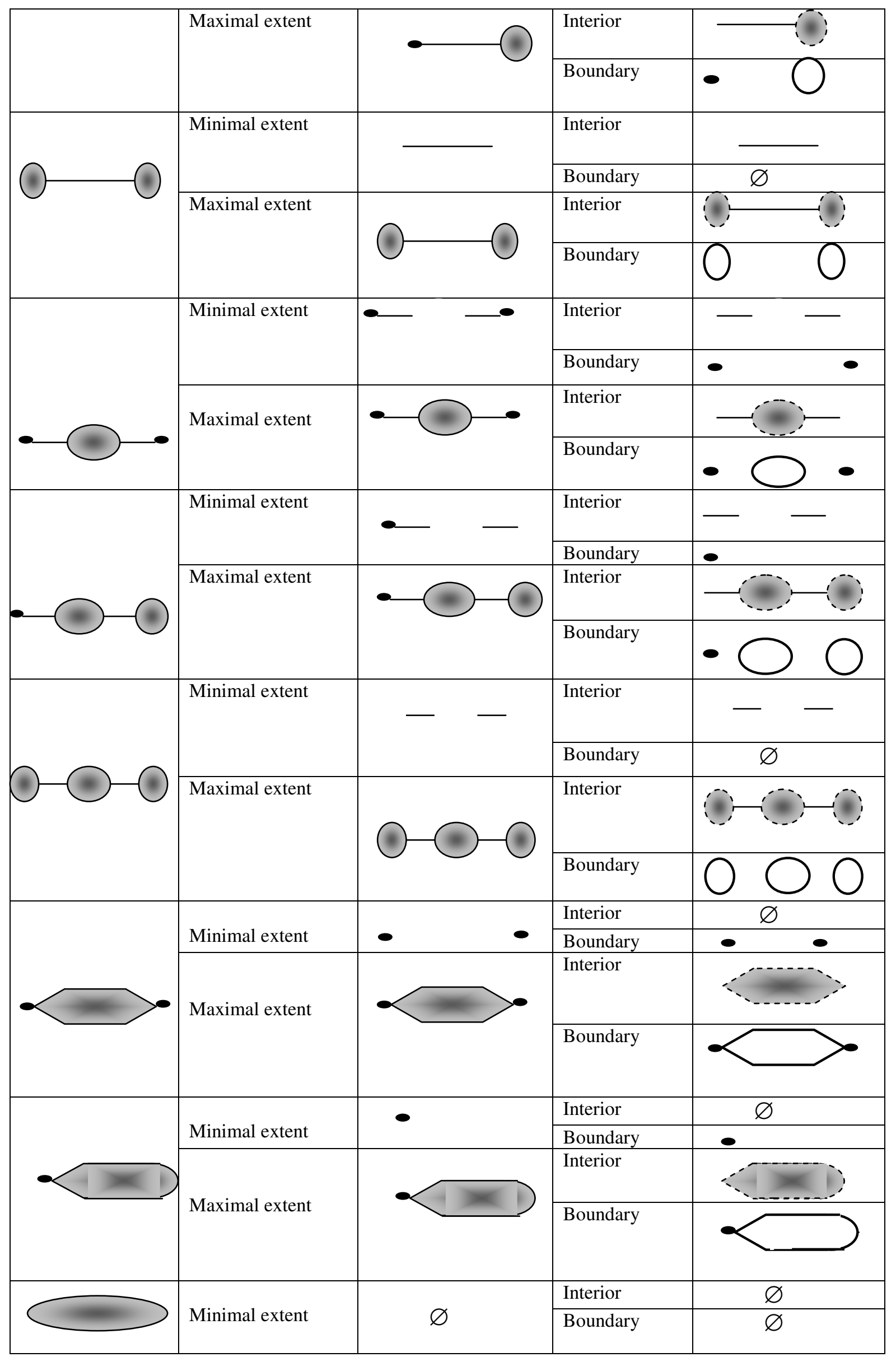




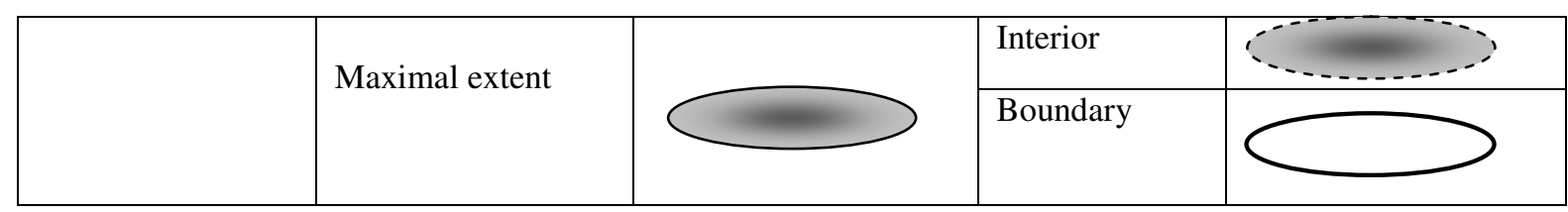

: The line does not belong to the interior

: Fuzzy point

: Crisp end point

Figure 12. Topological invariants according to the line fuzziness

More formally, a fuzzy line $\tilde{L}$ is the union of a maximal extent $\tilde{L}_{\max }$ and a minimal extent $\tilde{L}_{\min }$. In our approach, we look for the most expressive model possible in terms of specification of the topological relations. Consequently, we focus on the definition of the topological invariants for the maximal $\tilde{L}_{\max }$ and the minimal $\tilde{L}_{\min }$ extents. For each one, we distinguish an interior and a boundary that can be empty according to the configuration of the line (figure 12). From a point-set topology view point, a simple fuzzy line should verify the following conditions:

1- Each one-dimensional part of the simple fuzzy line is connected.

2- Each one-dimensional part of the simple fuzzy line is not self-intersecting.

3- Each one-dimensional part of the simple fuzzy line does not form a loop.

4- Each two-dimensional part verifies the conditions defined for a fuzzy point (section 3.1).

5- If the endpoints are fuzzy, they do not overlap with each other.

The three first conditions are those defined for a crisp line in the general point-set topology. Then, we apply these conditions to each linear part of the fuzzy line. The fourth condition requires that every fuzzy point on the interior or the endpoints of a line respect the topological conditions of a crisp region applied to a fuzzy point (section 3.1). The last condition is defined to eliminate any risk of self-intersection or loop configurations. Figure 13 shows some cases of lines that are invalid according to our model.

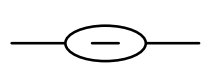

Non regular interior of maximal extent

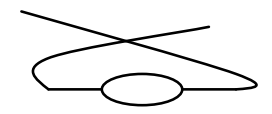

Self-intersecting line

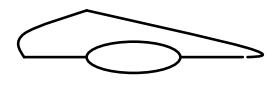

The line forms a loop

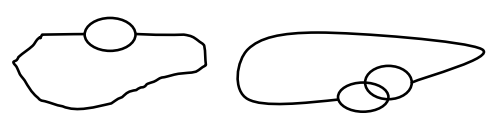

The endpoints can be identical

Figure 13. Examples of invalid lines

In the next cases, we give two examples of fuzzy lines:

- A strongly fuzzy line with only one crisp end point

The Bermuda triangle is a region in the Atlantic Ocean where some aircrafts and surface vessels have disappeared. We suppose that we have been appointed to redraw the trajectory of an engine that has disappeared. We will quickly discover that the only certain information is the last position of the engine before the communication interruption. In fact, this point refers to the minimal extent of the line. Far from this point, the trajectory can have any shape and position inside the fuzziness area. Indeed, our model is sufficiently expressive to represent this fuzziness case. 
- A fairly fuzzy line with two crisp endpoints

We suppose that an aircraft disappeared for some time from radar screens. After that, the communication with the aircraft returns to normal and the engine arrives at its destination. In this case, the aircraft trajectory is composed of two crisp endpoints. However, the interior is partially fuzzy, because the trajectory can take any unpredictable shape inside the dangerous zone.

\subsection{Fuzzy region}

A crisp region is a two-dimensional spatial object type in which the shape is typically composed of an interior, a boundary, and an exterior. For a region, shape fuzziness occurs when there is difficulty in precisely distinguishing between the interior and the exterior via a sharp boundary. From this perspective, shape fuzziness is generally correlated with the boundary, which can itself be crisp, partially fuzzy, or completely fuzzy. It is possible to draw a minimal spatial extent by considering the boundary to be as close as possible (i.e. it is drawn around the area which certainly belongs to the region). In the same way, a maximal spatial extent can be drawn by considering the boundary to be as far as possible (i.e. it is drawn around the area which contains all of points possibly belonging to the region). Figure 14 represents an example of a fuzzy region in which the boundary is partially fuzzy. The spatial extent of a fuzzy region is composed of a portion called the minimal extent (i.e., all of the points definitely belonging to the spatial object) and covered by a maximal extent (i.e., all of the points possibly belonging to the spatial object).

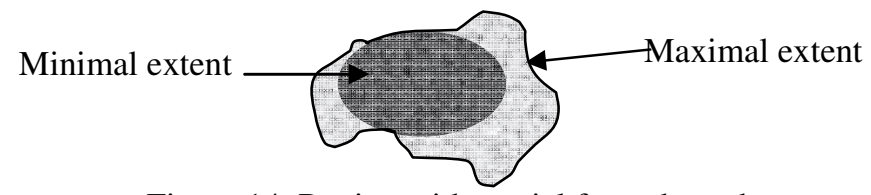

Figure 14. Region with partial fuzzy boundary

We consider that a simple fuzzy region is made up of two crisp regions: (1) the maximal extent, which can be "Equal", "Contains", or "Covers" (2) the minimal extent (figure 14). When the boundary is totally sharp (i.e. it does not contain any fuzzy point), the region is called completely crisp. This is a particular case of fuzzy region for which the maximal extent is equal to the minimal extent. In the second case, the region's boundary is fuzzy only in some locations. We speak about partially fuzzy regions, where the maximal extent covers the minimal extent. For example, a forest stand or a lake can have sharp boundaries (e.g., rocker borders for a lake and a total cut for a forest stand) and fuzzy boundaries (e.g., swamp borders for a lake) at the same time. The third case represents a typical fuzzy region for which the boundary is entirely fuzzy. For example, the boundary of a pollution zone is fuzzy everywhere since the pollution decreases from its kernel to the region's exterior. In the figure 15, we present an example of each of these three cases.

\begin{tabular}{|l|l|l|l|l|l|}
\hline Fuzzy region & Representation & \multicolumn{2}{|c|}{$\begin{array}{c}\text { Maximal and minimal } \\
\text { extents }\end{array}$} & \multicolumn{2}{c|}{$\begin{array}{c}\text { Topological invariants of } \\
\text { minimal and maximal extents }\end{array}$} \\
\cline { 3 - 6 } & & \multicolumn{2}{|c|}{ Interior } & Boundary \\
\hline $\begin{array}{l}\text { Region with } \\
\text { sharp boundary } \\
\text { (i.e., crisp } \\
\text { region) }\end{array}$ & $\begin{array}{l}\text { Minimal } \\
\text { extent }\end{array}$ & $\begin{array}{l}\text { Maximal } \\
\text { extent }\end{array}$ & & & \\
\cline { 3 - 6 } & & & & \\
\hline
\end{tabular}




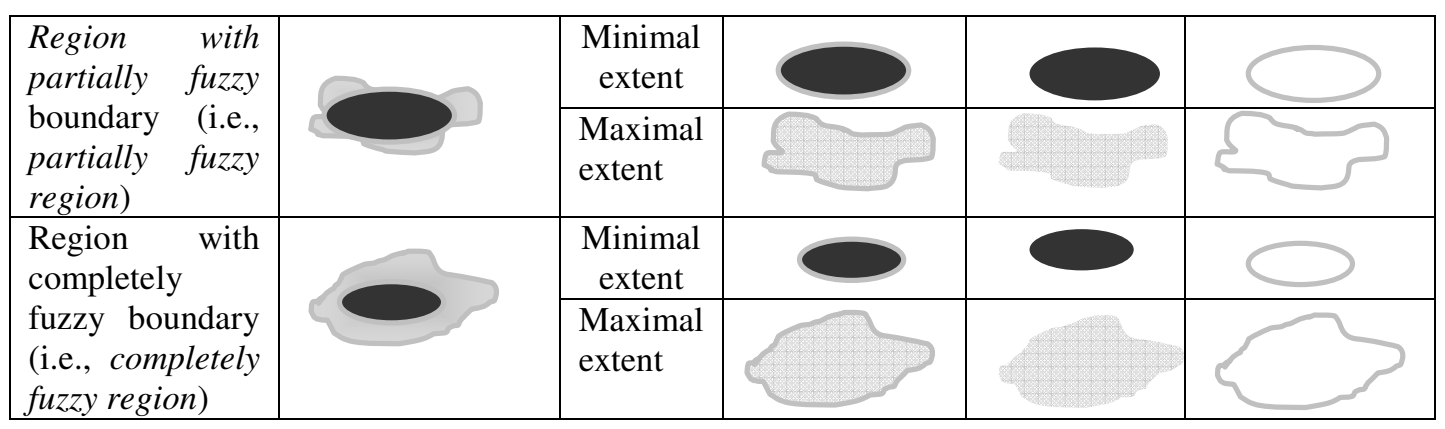

Figure 15. Fuzzy regions

Since the minimal and maximal extents are crisp regions, we distinguish three mutually disjoint topological invariants for each of them: an interior, a boundary, and an exterior. Thus, a fuzzy region $\tilde{A}$ is made up of six topological invariants: the interior of the minimal extent $\tilde{A}_{\text {min }}^{\circ}$, the boundary of the minimal extent $\partial \tilde{A}_{\min }$, the exterior of the minimal extent $\tilde{A}_{\text {min }}^{-}$, the interior of the maximal extent $\tilde{A}_{\max }^{\circ}$, the boundary of the maximal extent $\partial \tilde{A}_{\max }$, and the exterior of the maximal extent $\tilde{A}_{\max }^{-}$(figure 15).

- Definition 1: A simple fuzzy region $\tilde{A}$ is composed of two simple crisp regions $\tilde{A}_{\text {max }}$ and $\tilde{A}_{\text {min }}$, where $\operatorname{Equal}^{3}\left(\tilde{A}_{\text {max }}, \tilde{A}_{\text {min }}\right)$, Contains $\left(\tilde{A}_{\text {max }}, \tilde{A}_{\text {min }}\right)$, or Covers $\left(\tilde{A}_{\text {max }}, \tilde{A}_{\text {min }}\right) . \tilde{A}_{\text {min }}$ is the minimal extent of $\tilde{A}$, $\partial \tilde{A}_{\min }$ is the inner boundary of $\tilde{A}, \tilde{A}_{\max }$ is the maximal extent of $\tilde{A}$, and $\partial \tilde{A}_{\max }$ is the outer boundary of $\tilde{A} . \tilde{A}_{\min }$ is the set of points certainly belonging to $\tilde{A}$. However, the maximal extent $\tilde{A}_{\max }$ is the union of the minimal extent and the set of points possibly belonging to the fuzzy region.

The following conditions should be respected for any fuzzy region:

1- The closures of the maximal and the minimal extents are non-empty regular connected closed subsets.

2- The interiors of the maximal and minimal extents are non-empty regular open sets.

3- The boundaries and exteriors of the maximal and minimal extents are connected.

Figure 16 presents some examples of invalid fuzzy regions. In case (a), the region is invalid because its closure is non-regular, i.e. there is an isolated line that belongs to the closure. In case (b), the interior of the region is non-connected because it is composed of two disjoint subregions. In the case (c), the exterior does not respect the connectedness condition since the interior contains a hole.

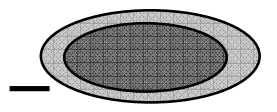

(a) Non-regular closed closure

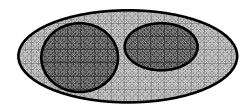

(b) Non-connected interior

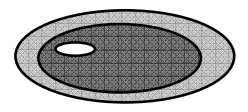

(c) Non-connected exterior

Figure 16. Examples of invalid fuzzy regions

This general definition covers the crisp regions occurring when Equal $\left(\tilde{A}_{\max }, \tilde{A}_{\text {min }}\right)$. Hereafter, we only focus on the typical fuzzy regions where Contains $\left(\tilde{A}_{\max }, \tilde{A}_{\min }\right) \operatorname{or} \operatorname{Covers}\left(\tilde{A}_{\max }, \tilde{A}_{\min }\right)$.

\footnotetext{
${ }^{3}$ The spatial relations (i.e., Equal, Contains, Covers) used in this definition are those defined in (Egenhofer and Herring 1990).
} 


\section{Topological relations between fuzzy spatial objects}

\subsection{Principles}

To identify the topological relations between two fuzzy objects, we interpret their maximal and minimal extents as independent crisp geometries. In fact, our methodology consists of identifying four specific topological relations between the minimal and maximal extents of the fuzzy spatial objects. For that, we define a 4-Intersection matrix containing the following four topological subrelations: $R_{l}\left(\tilde{A}_{\min }, \quad \tilde{B}_{\min }\right), \quad R_{2}\left(\tilde{A}_{\min }, \tilde{B}_{\max }\right), \quad R_{3}\left(\tilde{A}_{\max }, \quad \tilde{B}_{\min }\right), \quad$ and $R_{4}\left(\tilde{A}_{\max }, \widetilde{B}_{\max }\right)$ (see example in figure 17). The topological subrelations assigned to the matrix's cells are those defined in the 9-Intersection model (Egenhofer and Herring 1990). For example, if we study the topological relations between two fuzzy regions, each cell receives one of the eight possible topological relations between two simple crisp regions (i.e., Disjoint, Overlap, Meet, Equal, Contains, Inside, Covers, Covered by). Then, the 4-Intersection matrix corresponds to the following representation:

$$
\underset{\tilde{A}_{\max }}{\tilde{A}_{\min }}\left[\begin{array}{c}
\tilde{B}_{\text {max }} \\
\tilde{R}_{l}\left(\tilde{A}_{\min }, \tilde{B}_{\min }\right), R_{2}\left(\tilde{A}_{\min }, \tilde{B}_{\max }\right) \\
R_{3}\left(\tilde{A}_{\max }, \tilde{B}_{\min }\right), R_{4}\left(\tilde{A}_{\max }, \tilde{B}_{\max }\right)
\end{array}\right]
$$

Figure 17 shows the content of the matrix that describes the topological relation between a partially fuzzy region $\tilde{A}$ and a completely fuzzy region $\tilde{B}$. In the matrix (b), the letters $O$ and $C$ are used to denote the relations Overlap and Contains, respectively.

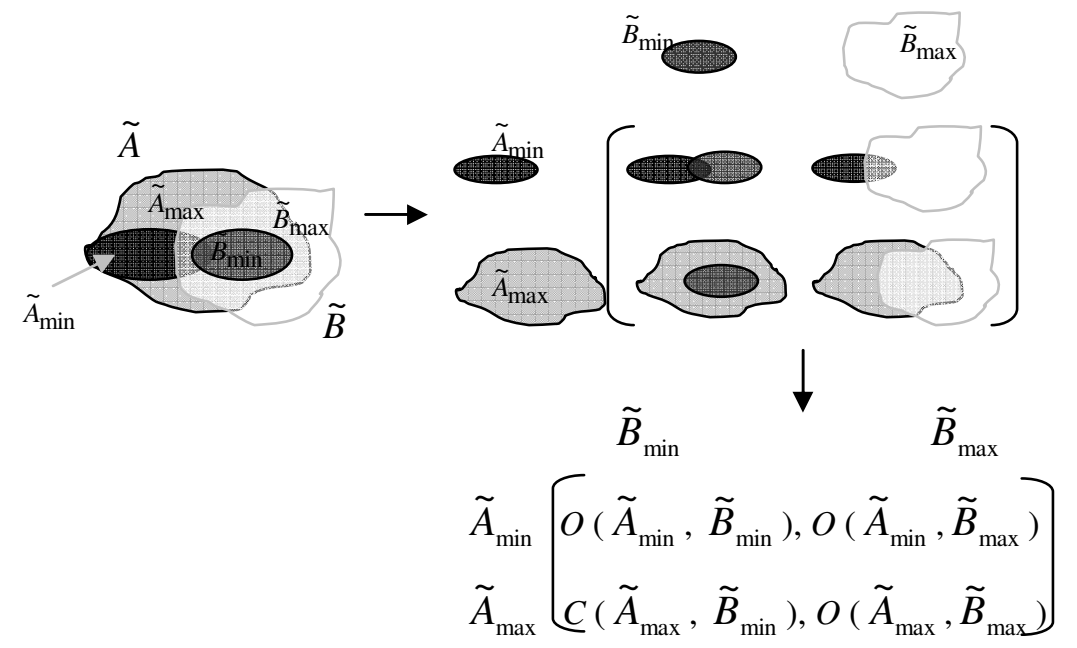

Figure 17. Description of the topological relation between two fuzzy regions: (a) visual content of the matrix, (b) formal identification of the relations between the minimal and maximal extents of the objects involved

The content of the matrix corresponds to the topological subrelations relating the minimal and maximal extents. We use the topological subrelation between the maximal extents $R_{4}\left(\tilde{A}_{\max }, \tilde{B}_{\max }\right)$ to label the global topological relation. For example, if $R_{4}\left(\tilde{A}_{\max }, \tilde{B}_{\max }\right)$ is Overlap, we consider that the fuzzy spatial objects globally Overlap each other. If $R_{4}\left(\tilde{A}_{\max }, \widetilde{B}_{\max }\right)$ is Contains, we consider that the global topological relation is Contains.

In figure 18, we present examples of the identification of topological relations between fuzzy spatial objects. The first example presents a description of the topological relation between two completely fuzzy regions $\tilde{A}$ and $\tilde{B}$. The second example concerns a fairly fuzzy line $\tilde{L}$ 
and a completely fuzzy region $\tilde{A}$. The third example shows the identification of the topological relation between two fairly fuzzy lines $\tilde{L}$ and $\tilde{K}$. Finally, the last example concerns a completely fuzzy region $\tilde{A}$ and a fuzzy point $\tilde{P}$.

\begin{tabular}{|c|c|}
\hline Spatial representation & Correspondent matrix \\
\hline$\tilde{B}$ & 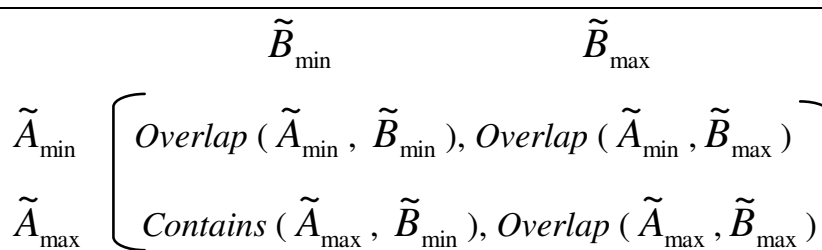 \\
\hline Global topological relation : Contains & 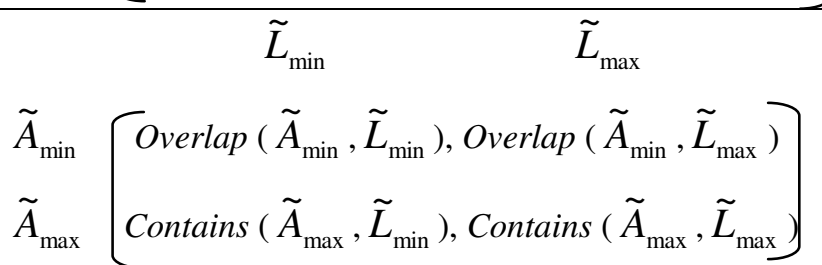 \\
\hline Global topological relation : Overlap & 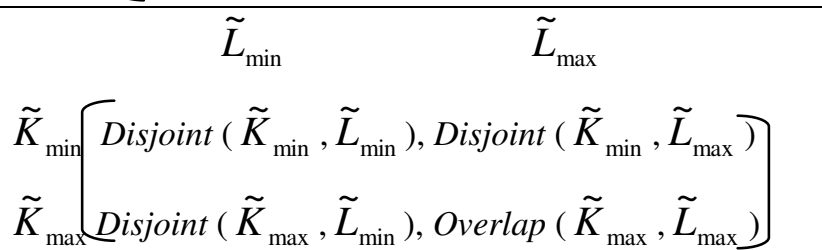 \\
\hline Global topological relation: Disjoint & $\begin{array}{c}\tilde{P}_{\max } \\
\tilde{A}_{\min }\left[\begin{array}{l}\left.\operatorname{Disjoint}\left(\tilde{A}_{\min }, \tilde{P}_{\max }\right)\right] \\
\tilde{A}_{\max } \operatorname{Disjoint}\left(\tilde{A}_{\max }, \tilde{P}_{\max }\right)\end{array}\right]\end{array}$ \\
\hline
\end{tabular}

Figure 18. Examples of identification of topological relations through a 4-Intersection matrix

\subsection{Topological relations between a fuzzy region and a crisp one}

In our approach, the 4-Intersection matrix highlights the subrelations that exist between the components of the fuzzy geometries. Indeed, this expressivity is highlighted when the maximal extent of the fuzzy spatial object is different from the minimal extent. In the other cases, some cells in the matrix will have the same values. For example, figure 19 shows a fuzzy region that overlaps a crisp region. The topological relation can be reduced to a 2Intersections matrix, because the region $\tilde{B}$ is crisp and so its minimal extent equals its maximal one. Hereafter, we do not study topological relations that involve crisp regions.

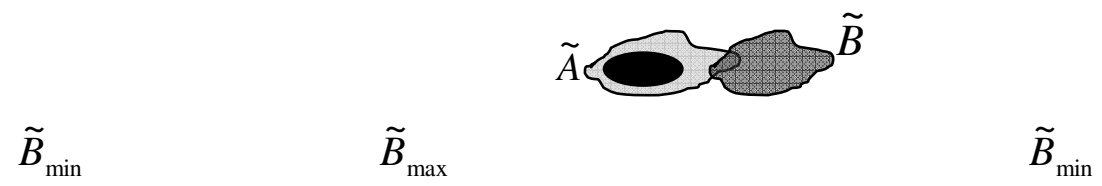

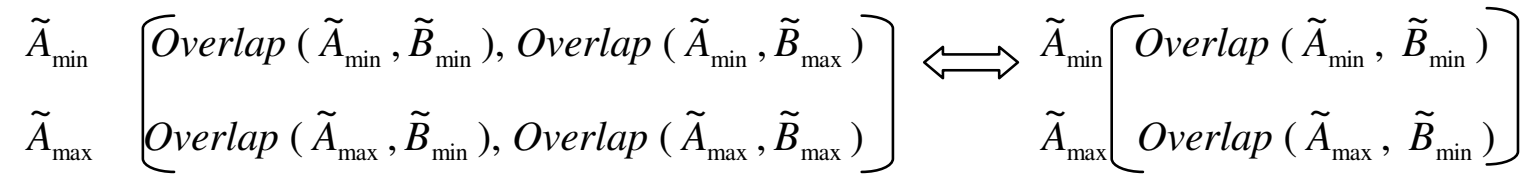

Figure 19. Example of a topological relation between a fuzzy region and a crisp region 
The values assigned to the different cells of the matrix should not be arbitrarily chosen. In general, the value of $R\left(\tilde{A}_{\max }, \widetilde{B}_{\max }\right)$ enforces the other values. In the next section, we study these aspects specifically for the topological relations between fuzzy regions.

\subsection{Topological relations between fuzzy regions}

Eight topological relations are possible between two simple crisp regions. By considering these as the possible values in the four cells of the matrix, there are $8^{4}=4096$ possible matrices. However, definition 1 imposes a condition mandating that the extents of a fuzzy region should be related by one of the following relations: Equal $\left(\tilde{A}_{\max }, \tilde{A}_{\min }\right)$, Contains $\left(\tilde{A}_{\text {max }}, \tilde{A}_{\text {min }}\right)$, or Covers $\left(\tilde{A}_{\text {max }}, \tilde{A}_{\text {min }}\right)$. Indeed, a 4-Intersection matrix cannot identify a topological relation between two fuzzy regions when this condition is violated. Thus, the contents of the matrix's cells are not independent. For example, if the maximal extents are disjoint, it is impossible for an Overlap to exist between the minimal extents (figure 20). In figure 20, the subrelation $O\left(\tilde{A}_{\text {min }}, \widetilde{B}_{\text {min }}\right)$ is grey to denote that is not allowed whereas $\boldsymbol{D}\left(\tilde{A}_{\text {min }}\right.$, $\tilde{B}_{\min }$ ) is black to show that is permitted. Consequently, several of the 4096 possible matrices are invalid because the dependency between the matrix's cells is not respected.

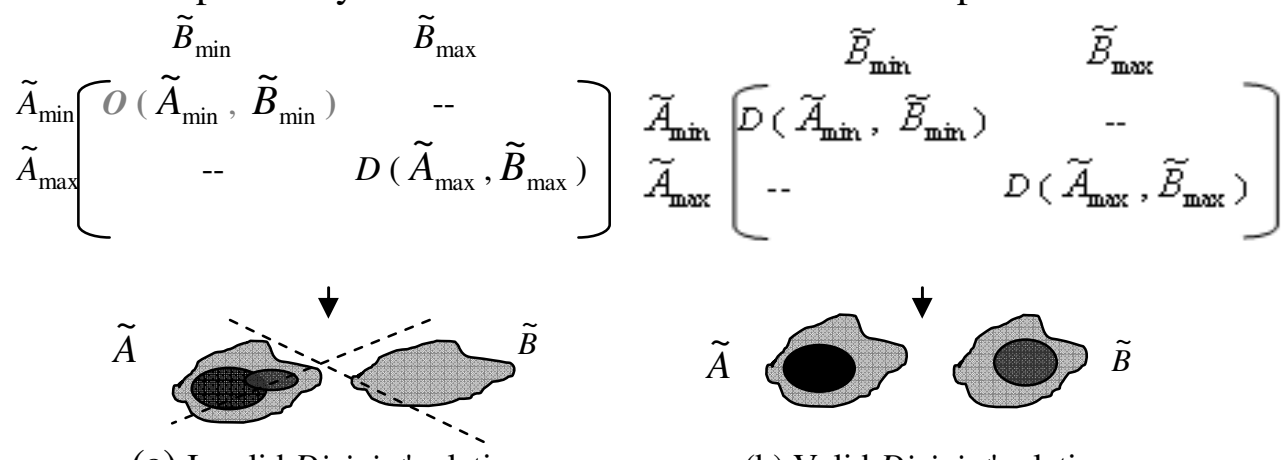

(a) Invalid Disjoint' relation

(b) Valid Disjoint' relation

Figure 20. Controlling the validity of a Disjoint relation

In order to enumerate the valid 4-Intersection matrices, we firstly studied the possible values in the other three cells for each of the eight possible values of $R\left(\tilde{A}_{\max }, \widetilde{B}_{\max }\right)$. For example, if Contains $\left(\tilde{A}_{\max }, \widetilde{B}_{\max }\right.$ ), the only possible relation between $\tilde{A}_{\max }$ and $\tilde{B}_{\min }$ is Contains; otherwise, the expected relation cannot respect the general definition of a fuzzy region. The figure 20 shows an example of an inconsistent matrix in which Disjoint $\left(\widetilde{A}_{\max }, \widetilde{B}_{\max }\right)$ and Contains ( $\left.\tilde{A}_{\text {min }}, \tilde{B}_{\text {min }}\right)$. This matrix is inconsistent because $R\left(\tilde{B}_{\text {max }}, \tilde{B}_{\text {min }}\right) \notin\{$ Contains, Covers, Equal $\}$. In the second step, we also fix the relation between $\tilde{A}_{\min }$ and $\tilde{B}_{\min }$ to deduce the possible values of $R\left(\tilde{A}_{\min }, \widetilde{B}_{\max }\right)$. For example, when Contains $\left(\tilde{A}_{\max }, \widetilde{B}_{\max }\right)$ and Contains $\left(\tilde{A}_{\min }, \widetilde{B}_{\min }\right), R$ ( $\tilde{A}_{\text {min }}, \tilde{B}_{\text {max }}$ ) should not be Meet or Equal. In this way, 31 rules (see table 4 in the appendix) are defined in order to ensure the consistency of matrices. In the rules' premises, we specify either $R\left(\tilde{A}_{\max }, \widetilde{B}_{\max }\right)$ or $\left(R\left(\tilde{A}_{\max }, \widetilde{B}_{\max }\right)\right.$ and $\left.R\left(\tilde{A}_{\text {min }}, \tilde{B}_{\text {min }}\right)\right)$. Then, we deduce the possible values in the remaining cells. In figure 20 , the matrix on the left is not valid because it requires the minimal extent to be disjoint to the minimal extent (i.e., the definition of the fuzzy region is not respected, because $R\left(\tilde{A}_{\max }, \widetilde{B}_{\max }\right)$ should be Contains, Covers, or Equal).

This study proves that only 242 topological relations are possible between two simple fuzzy regions (see the appendix). More specifically, only one matrix is valid when Disjoint 
$\left(\tilde{A}_{\max }, \tilde{B}_{\max }\right), 29$ matrices are valid when Contains $\left(\tilde{A}_{\max }, \tilde{B}_{\max }\right), 29$ for Inside $\left(\tilde{A}_{\max }, \tilde{B}_{\max }\right), 46$ for Covers $\left(\tilde{A}_{\max }, \widetilde{B}_{\max }\right), 46$ for Covered by $\left(\tilde{A}_{\max }, \widetilde{B}_{\max }\right), 65$ for Overlap $\left(\tilde{A}_{\max }, \widetilde{B}_{\max }\right), 4$ for Meet $\left(\tilde{A}_{\max }, \tilde{B}_{\max }\right)$, and 22 when Equal $\left(\tilde{A}_{\max }, \tilde{B}_{\max }\right)$. The topological relations are numbered from 1 to 242 according to the relation between $\tilde{A}_{\max }$ and $\widetilde{B}_{\max }$. Table 1 shows this numbering (see the appendix to explore the relations).

\section{Clustering of topological relations between fuzzy regions}

\subsection{Principles}

In our work, the proposed model is expressive in terms of the topological relations between fuzzy regions. In this context, 242 topological relations are distinguished. Consequently, the clustering of relations into larger groups of relations is an important step, because it is very difficult to keep in the mind this great number of relations. It is additionally very difficult to find a name for each one of these regions, and so the user will have difficulty choosing the appropriate operator in order to express a query or integrity constraint. Mark and Egenhofer (Mark and Egenhofer 1994) studied the clustering of the topological relations between simple crisp regions and simple crisp lines both through a formal basis and by taking into account cognitive aspects. Clementini and Di Felice (Clementini and Di Felice 1997) defined a topological distance to classify the approximate topological relations between regions with broad boundaries. In this way, they deduced 17 clusters that they represent in a conceptual neighborhood graph.

In our approach, most of the distinguished topological relations are not completely different from each other. For example, two simple fuzzy regions can weakly or completely overlap each other depending on the content of the matrix. In the first case, only the maximal extents overlap. In the second case, however, Overlap is the unique value in the matrix's four cells. Thus, it is possible to deduce the relation's fuzziness level from the content of the 4Intersection matrix. The objective of this section is to group the 242 topological relations into a limited number of clusters based on their fuzziness levels.

\subsection{Clustering results}

In section 4, we showed that the global topological relation is identified through a 4Intersection matrix that enumerates four subrelations. Thus, a topological relation becomes possible if it appears at least once in the matrix. This possibility increases according to the number of similar subrelations. For example, a Covers topological relation in which Covers ( $\left.\tilde{A}_{\max }, \tilde{B}_{\max }\right)$ and Covers $\left(\tilde{A}_{\text {min }}, \tilde{B}_{\text {min }}\right)$ is stronger than another where only Covers $\left(\tilde{A}_{\max }, \tilde{B}_{\max }\right)$. Because there are eight possible values for the matrix's cells, we distinguish eight basic clusters that we call: DISJOINT, CONTAINS, INSIDE, COVERS, COVERED BY, EQUAL, MEET, and OVERLAP. Each cluster contains all of the topological relations for which at least one of the four subrelations has the same name. For example, figure 21 shows a topological relation that belongs to the following clusters: DISJOINT, CONTAINS, and COVERS. Nevertheless, it belongs to the DISJOINT cluster more strongly than to the CONTAINS and COVERS clusters. 


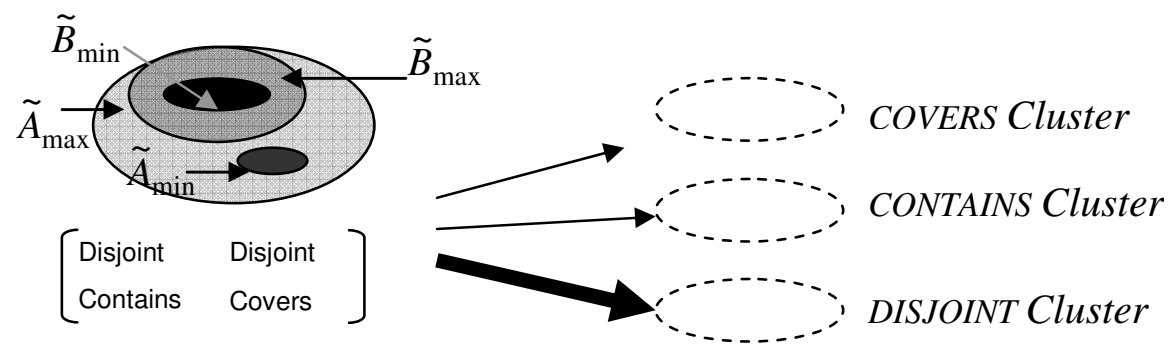

Figure 21. Example of clustering of a topological relation

For each one of the eight basic clusters, we identify four levels of relation membership: (1) completely, (2) strongly, (3) fairly, and (4) weakly (table 2). A topological relation belongs to the cluster completely when the four subrelations are similar. It belongs to the cluster strongly when only three subrelations have the same name as the cluster. The level labeled fairly contains all relations for which two subrelations have the same name as the cluster. Finally, the level called weakly contains the relations for which only one subrelation has the same name as the cluster. Figure 22 presents some relations that belong to different levels of CONTAINS and DISJOINT clusters, respectively, according to the contents of their correspondent matrices.

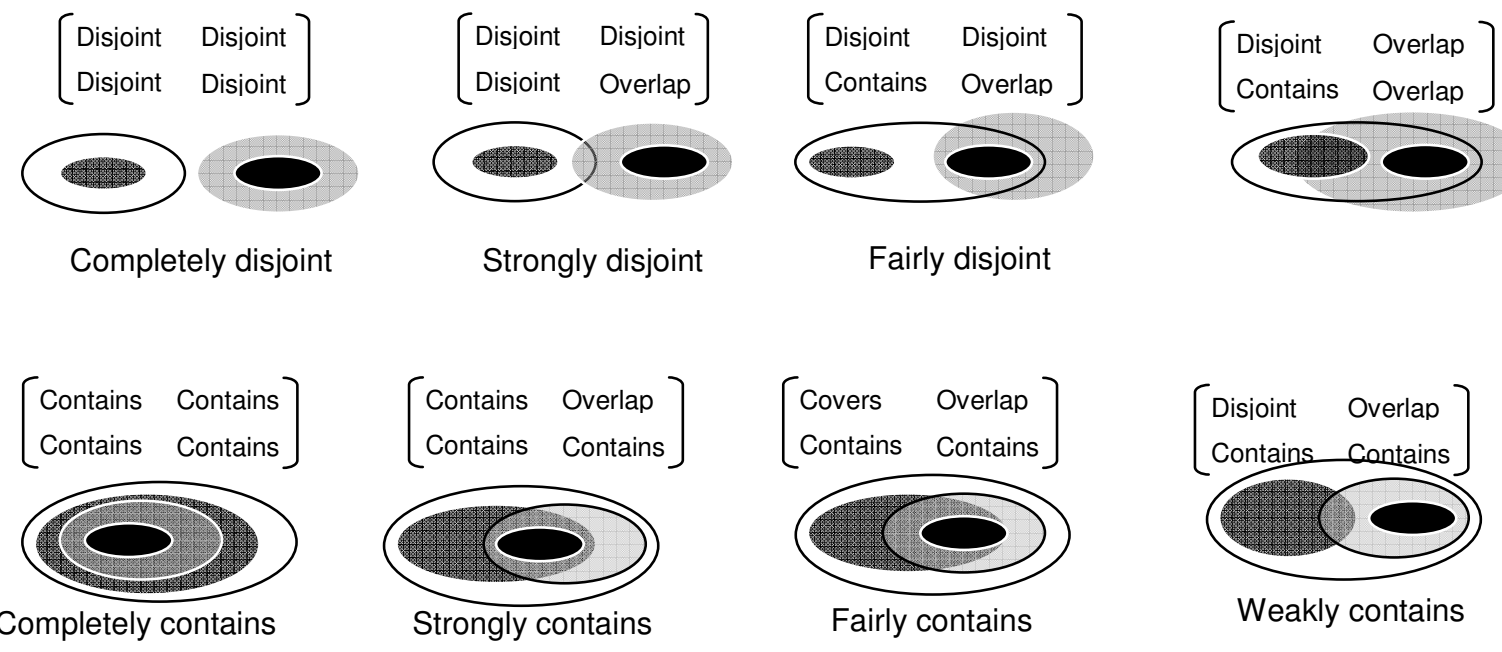

Figure 22. Evaluation of topological relation's fuzziness

\subsection{Overlapping clusters}

The main result of this clustering process is a hierarchical classification of the topological relations (figure 23). The top level is made up of eight basic clusters that each contains typically four levels: completely, strongly, fairly, and weakly. The resulting 32 subclusters overlap each other because a topological relation typically belongs to different levels of 1,2 , 3 , or 4 clusters at the same time. For example, topological relation number 56 (see the appendix and the table 2) belongs fairly to the CONTAINS cluster and weakly to the COVERS and INSIDE clusters. The bottom level of the classification contains the 242 topological relations that appear in different subclusters. 


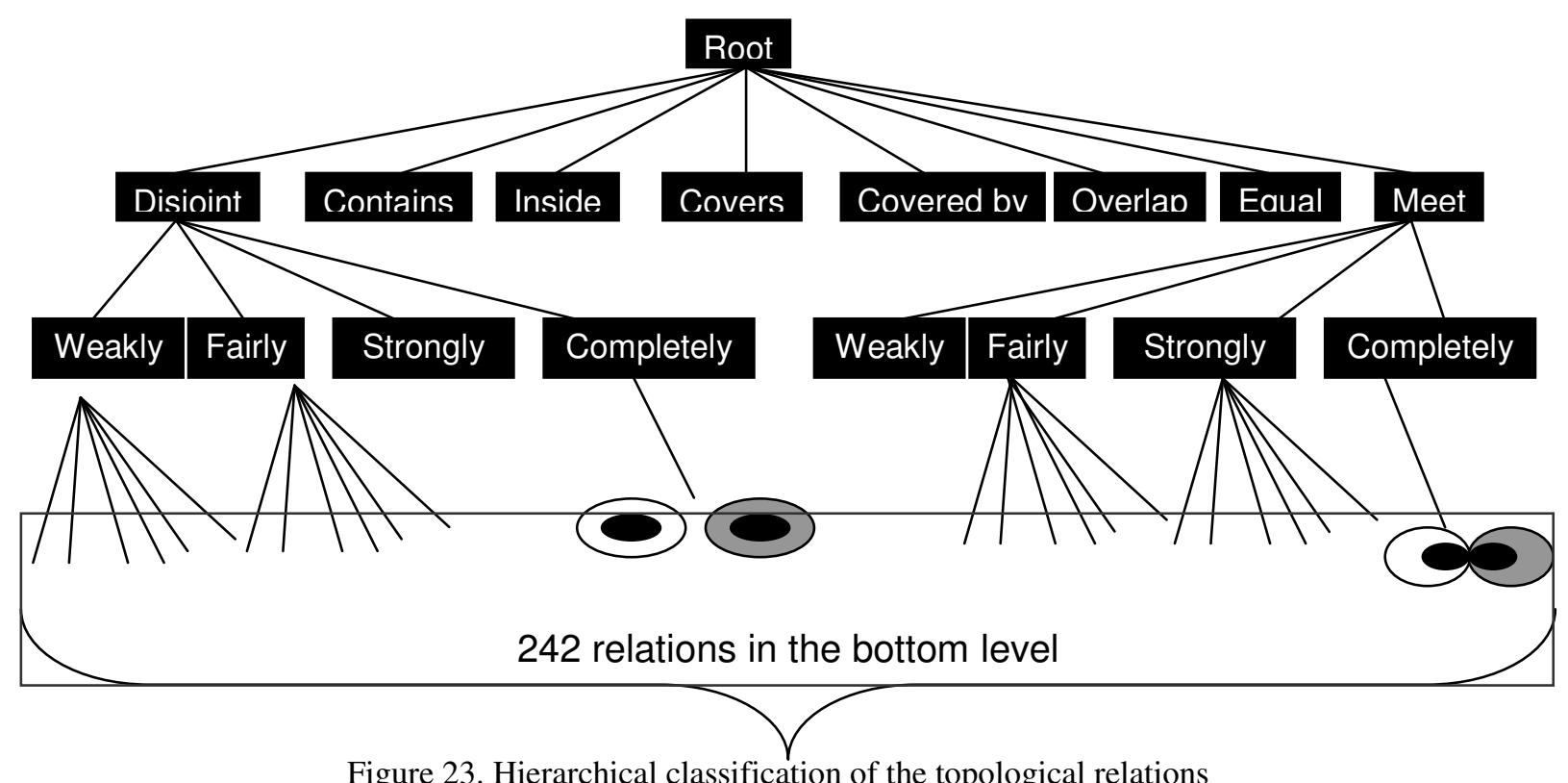

Figure 23. Hierarchical classification of the topological relations

\section{Specification of spatial queries and integrity constraints}

In the previous sections, we presented a framework for identifying topological relations between fuzzy regions. Because it uses the 9-Intersection model (Egenhofer and Herring 1990), our model can be easily integrated in a spatial database system. Indeed, the SQL language can be extended in order to retrieve fuzzy regions based on the qualitative information given by the user regarding their topological relation. In fact, a topological relation between two fuzzy regions can be recognized through the combination of four crisp spatial operators. For example, relation number 56 corresponds to (Disjoint, Disjoint, Contains, Covers). Hereafter, we suppose that we integrated our spatial model in a relational engine in order to give an example of its possible use in spatial queries involving fuzzy regions. We suppose that the spatial database stores pollution zones, which are represented as fuzzy regions. In the first query example, the user gives a coarse description of the topological relation when he introduces the specification fairly DISJOINT. The query's results should contain the pollution zones related to zone $A$ by a topological relation belonging to this subcluster. In the second example, the query is more specific because the user identifies all topological subrelations that relate $\left(\tilde{A}_{\min }, \widetilde{B}_{\min }\right),\left(\tilde{A}_{\min }, \widetilde{B}_{\max }\right),\left(\tilde{A}_{\max }, \widetilde{B}_{\min }\right)$, and $\left(\tilde{A}_{\max }, \widetilde{B}_{\max }\right)$. The third example shows another use of our model, in which it is possible to display the different fuzziness levels of a relation (e.g., Overlap) that occurs between two fuzzy regions (cf. table 3 ). Table 3 shows a possible result for the query presented in example 3.

\section{Example 1: Select Pollution_Zone.geometry From Pollution_Zone Where Fuzzy_Relate (pollution_zone.geometry, A.geometry, fairly DISJOINT);}

\section{Example 2: Select Pollution_Zone.geometry From Pollution_Zone Where Fuzzy_Relate (Pollution_Zone.geometry, A.geometry, Disjoint, Meet, Contains, Contains);}

Example 3: Select P1.id, P2.id, determine (P1.geometry, P2.geometry, "Overlap") From Pollution_Zone P1, P2 Where P1.id <>P2.id;

In the same way, it is possible to use the model to formally express spatial integrity constraints in a fuzzy context. For example, let the constraint saying that 'two different lakes 
can be only fairly meet or completely disjoint'. This constraint can be formally expressed by integrating new spatial operators (e.g., completely Contains, weakly Covers, etc.) in a formal constraint language like the Object Constraint Language (OCL) (Duboisset et al. 2007). The database storing the lakes is consistent only if the topological relations between the different entities belong to fairly MEET or completely DISJOINT subclusters (see example 4).

\section{Example 4: Context Lake inv: \\ Lake.allInstances $\rightarrow$ forAll (a, bl a<>b implies fairly $\boldsymbol{M E E T}(\mathrm{a}, \mathrm{b})$ or completely DISJOINT(a,b));}

\section{Discussion}

Clementini and Di Felice (Clementini and Di Felice 1997) propose an extension of the 9Intersection model (Egenhofer and Herring 1990) that uses a broad boundary to replace the sharp boundary. In this approach, 44 topological relations are distinguished between two regions with broad boundaries. By considering topological distance, Clementini and Di Felice (Clementini and Di Felice 1997) draw a conceptual neighborhood graph that shows similarity degrees between relations classified into 17 clusters. The main advantage of this approach is the ability to support a coarser spatial reasoning involving regions with broad boundaries. When the needs are more specific, it becomes more difficult to use this model. Furthermore, the identification of a broad boundary as a two-dimensional topological invariant requires respecting consistency conditions related to closeness and connectedness. Xinming (Xinming 2004) presents a more expressive model than that of (Clementini and Di Felice 1997), because he decomposes the large boundary into the boundary's interior and the boundary's boundary. Based on this definition, Xinming (Xinming 2004) presents another extension of 9Intersection model, in which topological relations are identified through a $4 * 4$-Intersection matrix. He distinguishes 152 topological relations presented as variants of the 44 relations from (Clementini and Di Felice 1997). Nonetheless, this model does not distinguish between the boundaries of the minimal and maximal extents. Thus, many topological relations cannot be distinguished. Moreover, regions with partially fuzzy boundaries (figure 11) are considered invalid and cannot be presented through this model. In our approach, we resolve this problem by considering a simple fuzzy region as a maximal extent and a minimal extent, in which either Equal $\left(\tilde{A}_{\max }, \tilde{A}_{\min }\right)$ (i.e., a crisp region) or Contains $\left(\tilde{A}_{\max }, \tilde{A}_{\min }\right)$ or Covers $\left(\tilde{A}_{\max }, \tilde{A}_{\min }\right)$. We do not define a large boundary as a topological invariant of fuzzy regions. Our main motivations for adopting this framework are (1) to consider partially fuzzy regions and (2) to present a more expressive model in terms of the identification of topological relations between simple fuzzy regions. In this work, topological relations are specified through a 4-Intersection matrix that enumerates the topological subrelations between $\left(\tilde{A}_{\text {min }}\right.$, $\left.\widetilde{B}_{\min }\right),\left(\tilde{A}_{\min }, \tilde{B}_{\max }\right),\left(\tilde{A}_{\max }, \widetilde{B}_{\text {min }}\right)$, and $\left(\tilde{A}_{\max }, \widetilde{B}_{\max }\right)$. The topological relations used here are those defined by the 9-Intersection model (Egenhofer and Herring 1990). We distinguish 242 topological relations that we classify into 8 overlapping basic clusters. Each cluster has four membership levels (or subclusters): completely, strongly, fairly, and weakly. Nevertheless, our model is not able to quantify the gradual change inside the maximal extent in the same way as the fuzzy approaches do (Zhan 1997, Schneider 2001, Dilo 2006).

The Egg-Yolk model (Cohn and Gotts 1996) was our main inspiration to develop this framework for identifying topological relations. However, there are some fundamental differences between our model and that of (Cohn and Gotts 1996). For instance, the topological relations used in (Cohn and Gotts 1996) are those defined in the RCC-5 model (Randell and Cohn 1989, Cohn et al. 1997). In contrast, our model is based on point-set 
topology, in which points and lines are considered as the basic crisp spatial object types. In (Cohn and Gotts 1996), a conceptual neighborhood graph was drawn with 44 topological relations are classified into 13 clusters. In our model, we define a hierarchical classification based on the content of the matrices we use to identify the topological relations.

\section{Conclusions and future work}

Shape fuzziness is an inherent property of many spatial objects like lakes, valleys, and mountains. In GIS and spatial databases, it is a general practice to neglect shape fuzziness and formally represent fuzzy spatial objects as crisp geometries. Using such inappropriate representations can provide a source of spatial data quality degradation, because the reliability of spatial data is decreased. With emergence of prediction applications, data integration, and strategic decisional needs, researchers are increasingly more motivated to propose different methods for the formal representation of spatial fuzziness. A review of the literature regarding this topic proves that existing exact models do not permit the representation of partially fuzzy objects. For such objects, shape fuzziness partially characterizes one or several of its topological invariants. For example, a lake can have rocker and swamp banks at the same time; the boundary is fuzzy only for the swamp part. In this work, we have proposed an exact model in order to represent spatial objects that can be: crisp, partially fuzzy, or completely fuzzy. We have considered this categorization of fuzziness during the identification of topological relations.

More specifically, this paper contributes in three main ways. Based on point-set topology, we firstly define three basic fuzzy spatial object types: fuzzy point, fuzzy line, and fuzzy region. Each one of them is typically defined as a minimal extent $\tilde{A}_{\text {min }}$ and a maximal extent $\tilde{A}_{\max }$, and these extents must verify some topological conditions in order to be valid. This model permits the representation of partially fuzzy spatial objects considered as invalid in the existing models of (Clementini and Di Felice 1997, Xinming 2004, Reis et al. 2006). Then, we identify a topological relation through use of a 4-Intersection matrix that permits the enumeration of four subrelations: $R_{1}\left(\tilde{A}_{\min }, \tilde{B}_{\min }\right), R_{2}\left(\tilde{A}_{\min }, \tilde{B}_{\max }\right), R_{3}\left(\tilde{A}_{\max }, \tilde{B}_{\min }\right)$, and $R_{4}$ $\left(\tilde{A}_{\max }, \tilde{B}_{\max }\right)$. By using this formalism for simple fuzzy regions, 242 relations can be distinguished (cf. appendix). In order to retain our propositions useful in practice, we propose the clustering of these topological relations. A topological relation can belong to one or several clusters with various levels of membership: completely, strongly, fairly, and weakly. The objective of this qualitative clustering is to improve the specification of spatial queries and integrity constraints involving fuzzy spatial objects.

The main perspective of this work aims to improve the logical consistency of spatial databases involving fuzzy spatial objects. More specifically, we are interested in the specification of integrity constraints in spatial databases storing fuzzy spatial objects. We hope to identify both integrity constraint categories and the requirements for their formal expression. The framework presented earlier can provide a basis for the extension of a formal constraint language like OCL (Duboisset et al. 2007) to express tolerant integrity constraints for fuzzy spatial objects. In the same way, decisional applications require the integration of spatial data from heterogeneous sources before they are stored in a spatial data warehouse (Bédard et al. 2007). The main difficulty lies in choosing one of the available representations. We suggest merging the different representations in such way that the result looks like a fuzzy spatial object. The fuzzy integrity constraints can be used to increase the logical consistency of such data. 


\section{Appendix: $\mathbf{2 4 2}$ topological relations between fuzzy regions and required rules to deduce them}

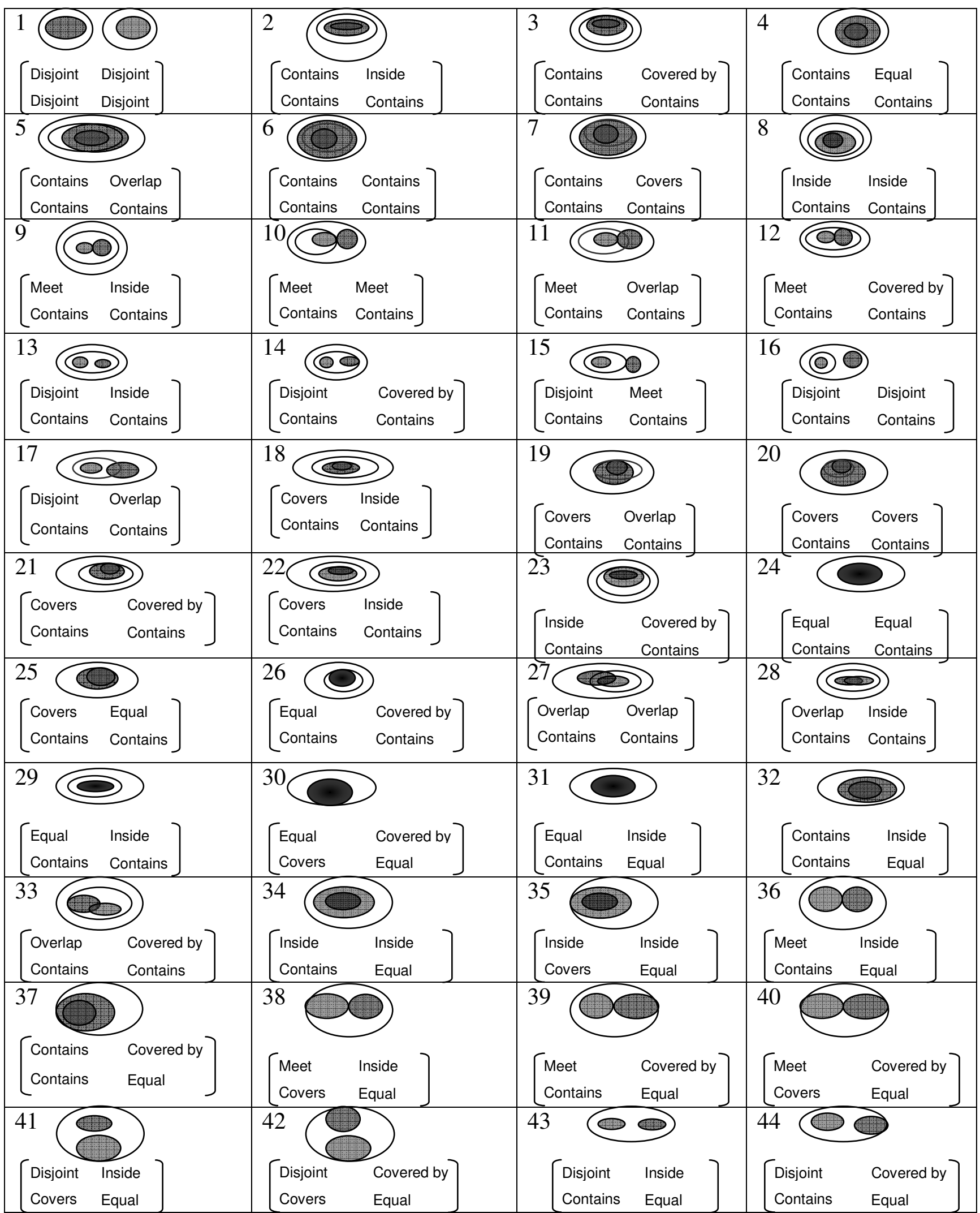




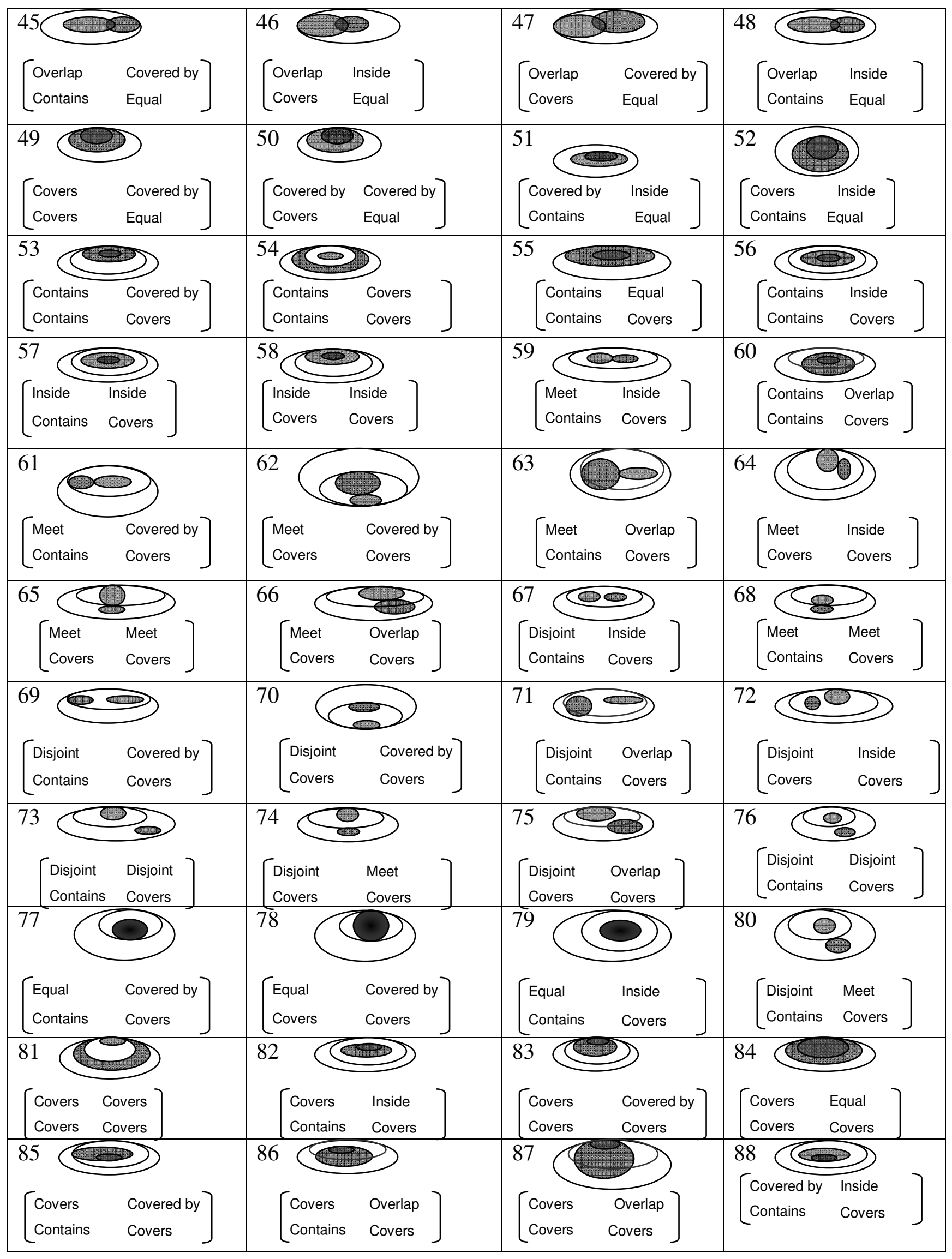




\begin{tabular}{|c|c|c|c|}
\hline $\begin{array}{l}{\left[\begin{array}{ll}\text { Covered by } & \text { Covered by } \\
\text { Covers } & \text { Covers }\end{array}\right]}\end{array}$ & $\begin{array}{ll}90 & \begin{array}{ll}\text { Covered by } & \text { Inside } \\
\text { Covers } & \text { Covers }\end{array}\end{array}$ & $\begin{array}{l}91010 \\
{\left[\begin{array}{ll}\text { Covers } & \text { Equal } \\
\text { Contains } & \text { Covers }\end{array}\right.}\end{array}$ & 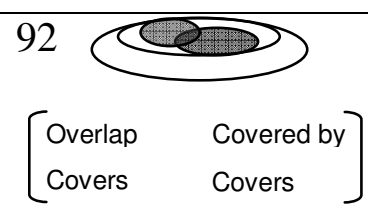 \\
\hline $\begin{array}{l}93 \bigcirc \bigcirc \\
{\left[\begin{array}{ll}\text { Overlap } & \text { Inside } \\
\text { Contains } & \text { Covers }\end{array}\right.}\end{array}$ & {$\left[\begin{array}{ll}\text { Overlap } & \text { Covered by } \\
\text { Contains } & \text { Covers }\end{array}\right]$} & {$\left[\begin{array}{ll}\text { Covered by } & \text { Covered by } \\
\text { Contains } & \text { Covers }\end{array}\right.$} & 96 \\
\hline$\left[\begin{array}{ll}\text { Overlap } & \text { Overlap } \\
\text { Covers } & \text { Covers }\end{array}\right.$ & $\begin{array}{ll}98 \\
\begin{array}{ll}\text { Overlap } & \text { Inside } \\
\text { Covers } & \text { Covers }\end{array}\end{array}$ & $\begin{array}{l}99 \\
{\left[\begin{array}{ll}\text { Inside } & \text { Inside } \\
\text { Covers } & \text { Covered by }\end{array}\right]}\end{array}$ & $\begin{array}{l}100 \\
{\left[\begin{array}{ll}\text { Inside } & \text { Inside } \\
\text { Covered by } & \text { Covered by }\end{array}\right]}\end{array}$ \\
\hline $\begin{array}{ll}\text { Inside } & \text { Inside } \\
\text { Equal } & \text { Covered by }\end{array}$ & $\begin{array}{l}{\left[\begin{array}{ll}\text { Inside } & \text { Inside } \\
\text { Contains } & \text { Covered by }\end{array}\right]} \\
\text { Pand }\end{array}$ & 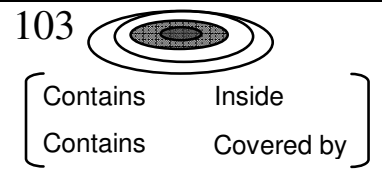 & {$\left[\begin{array}{ll}\text { Contains } & \text { Covered by } \\
\text { Contains } & \text { Covered by }\end{array}\right]$} \\
\hline Dovered by & $\begin{array}{l}\text { Inside } \\
\text { Overlap }\end{array}$ & {$\left[\begin{array}{ll}\text { Meet } & \text { Inside } \\
\text { Covers } & \text { Covered by }\end{array}\right]$} & $\begin{array}{l}\text { Meet } \\
\text { Covers }\end{array}$ \\
\hline Meet 109 & $\underbrace{110}_{\left.\begin{array}{ll}\text { Meet } & \text { Covered by } \\
\text { Contains } & \text { Covered by }\end{array}\right]}$ & 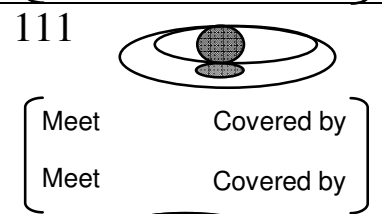 & {$\left[\begin{array}{l}\text { Meet } \\
\text { Overlap }\end{array}\right.$} \\
\hline $\begin{array}{l}113 \bigcirc 000 \\
\begin{array}{ll}\text { Disjoint } & \text { Inside } \\
\text { Contains } & \text { Covered by }\end{array} .\end{array}$ & Inside & $\begin{array}{ll}{\left[\begin{array}{ll}\text { Disjoint } & \text { Inside } \\
\text { Covers } & \text { Covered by }\end{array}\right]} \\
0\end{array}$ & Covered by \\
\hline$\underbrace{117}_{\begin{array}{ll}\text { Disjoint } \\
\text { Overlap } & \text { Inside } \\
\text { Covered by }\end{array}}$ & {$\left[\begin{array}{ll}\text { Disjoint } & \text { Covered by } \\
\text { Contains } & \text { Covered by }\end{array}\right]$} & $\left.\begin{array}{ll}\text { Disjoint } & \text { Covered by } \\
\text { Disjoint } & \text { Covered by }\end{array}\right]$ & $\sum_{\substack{\text { Disjoint } \\
\text { Meet }}}^{120}$ \\
\hline $\begin{array}{l}\text { Disjoint } \\
\text { Overlap }\end{array}$ & $\begin{array}{ll}\text { Disjoint } & \begin{array}{l}\text { Inside } \\
\text { Disjoint } \\
\text { Covered by }\end{array}\end{array}$ & {$\left[\begin{array}{l}\text { Equal } \\
\text { Covers }\end{array}\right.$} & {$\left[\begin{array}{ll}\begin{array}{l}\text { Equal } \\
\text { Covers }\end{array} & \begin{array}{l}\text { Covered by } \\
\text { Covered by }\end{array}\end{array}\right.$} \\
\hline$\left[\begin{array}{ll}\left.\begin{array}{ll}\text { Equal } & \text { Inside } \\
\text { Contains } & \text { Covered by }\end{array}\right] \\
\end{array}\right.$ & $\begin{array}{ll}\begin{array}{ll}\text { Disjoint } & \text { Inside } \\
\text { Meet } & \text { Covered by }\end{array} \\
\end{array}$ & {$\left[\begin{array}{ll}\text { Covered by } & \text { Covered by } \\
\text { Covered by } & \text { Covered by }\end{array}\right]$} & $\begin{array}{l}{\left[\begin{array}{ll}\text { Covered by } & \text { Inside } \\
\text { Contains } & \text { Covered by }\end{array}\right.}\end{array}$ \\
\hline$\left[\begin{array}{ll}\text { Covered by } \\
\text { Covers } & \text { Covered by } \\
\text { Covered by }\end{array}\right]$ & Coverlap & $\begin{array}{ll}\begin{array}{l}\text { Covered by } \\
\text { Equal }\end{array} & \begin{array}{l}\text { Covered by } \\
\text { Covered by }\end{array}\end{array}$ & $\begin{array}{ll}\begin{array}{l}\text { Covered by } \\
\text { Covers }\end{array} & \text { Inside } \\
\end{array}$ \\
\hline 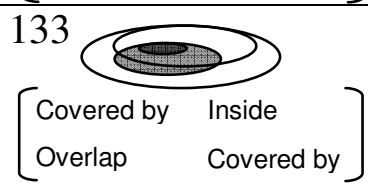 & 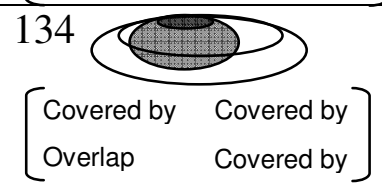 & {$\left[\begin{array}{ll}\begin{array}{l}\text { Covers } \\
\text { Contains }\end{array} & \text { Covered by }\end{array}\right.$} & Covered by \\
\hline
\end{tabular}




\begin{tabular}{|c|c|c|c|}
\hline $\begin{array}{ll}{\left[\begin{array}{ll}\text { Covers } & \text { Covered by } \\
\text { Contains } & \text { Covered by }\end{array}\right]} \\
137\end{array}$ & $\begin{array}{l}138 \\
{\left[\begin{array}{ll}\text { Covered by } \\
\text { Equal }\end{array}\right.} \\
\text { Covered by }\end{array}$ & {$\left[\begin{array}{ll}\text { Overlap } & \text { Covered by } \\
\text { Covers } & \text { Covered by }\end{array}\right]$} & {$\left[\begin{array}{ll}\begin{array}{l}\text { Overlap } \\
\text { Contains }\end{array} & \text { Inside } \\
\text { Covered by }\end{array}\right.$} \\
\hline$\left[\begin{array}{ll}\text { Overlap } & \text { Inside } \\
\text { Covers } & \text { Covered by }\end{array}\right]$ & {$\left[\begin{array}{ll}\text { Covers } & \text { Inside } \\
\text { Covers } & \text { Covered by }\end{array}\right.$} & Overlap $\begin{array}{c}\text { Inside } \\
\text { Overlap }\end{array}$ & $\begin{array}{ll}\text { Overlap } & \text { Covered by } \\
\text { Overlap } & \text { Covered by }\end{array}$ \\
\hline$\overbrace{\begin{array}{ll}\text { Equal } \\
\text { Contains }\end{array}} \begin{array}{l}\text { Inside } \\
\text { Inside }\end{array}$ & 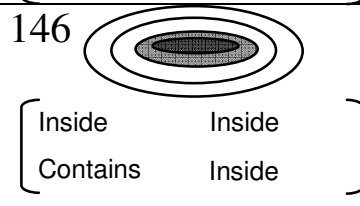 & $\begin{array}{ll}\begin{array}{l}\text { Inside } \\
\text { Covers }\end{array} & \text { Inside } \\
\text { Inside }\end{array}$ & $\begin{array}{ll}148 & \text { Inside } \\
\text { Inside } & \text { Inside } \\
\text { Equal } & 0\end{array}$ \\
\hline $\begin{array}{ll}\text { Inside } & \text { Inside } \\
\text { Overlap } & \text { Inside }\end{array}$ & $\begin{array}{l}150>\begin{array}{ll}\text { Inside } \\
\text { Inside } \\
\text { Inside }\end{array} \\
\end{array}$ & $\begin{array}{lll}\text { Inside } & \text { Inside } \\
\text { Covered by } & \text { Inside }\end{array}$ & $\begin{array}{ll}\begin{array}{l}\text { Contains } \\
\text { Contains }\end{array} & \begin{array}{l}\text { Inside } \\
\text { Inside }\end{array} \\
\end{array}$ \\
\hline $\begin{array}{l}153 \text { Meet } \\
\begin{array}{ll}\text { Mnside } \\
\text { Contains } & \text { Inside }\end{array}\end{array}$ & $\begin{array}{ll}\text { Meet } & \text { Inside } \\
\text { Meet } & \text { Inside }\end{array}$ & 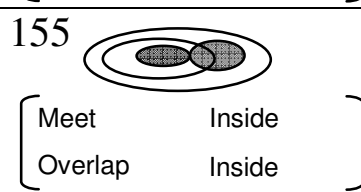 & $\begin{array}{ll}\text { Meet } & \text { Inside } \\
\text { Covers } & \text { Inside }\end{array}$ \\
\hline @ & $\begin{array}{l}158 \bigcirc \bigcirc 0 \\
{\left[\begin{array}{ll}\text { Disjoint } & \text { Inside } \\
\text { Disjoint } & \text { Inside }\end{array}\right.}\end{array}$ & 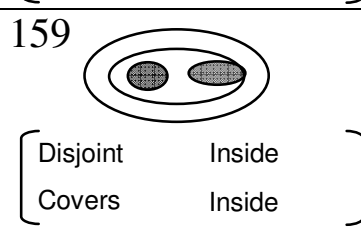 & 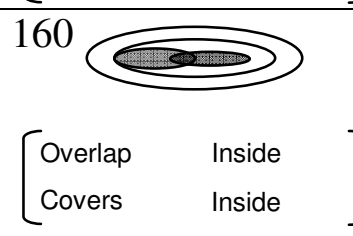 \\
\hline $\begin{array}{ll}161 & \text { Inside } \\
\text { Misjoint } & \text { Inside } \\
\text { Meet } & \end{array}$ & $\begin{array}{ll}\text { Disjoint } & \text { Inside } \\
\text { Overlap } & \text { Inside }\end{array}$ & $\begin{array}{l}{\left[\begin{array}{ll}\text { Covered by } & \text { Inside } \\
\text { Contains } & \text { Inside }\end{array}\right.}\end{array}$ & $\begin{array}{l}164>\begin{array}{ll}\text { Covered by } \\
\text { Onside }\end{array} \\
\text { Inside }\end{array}$ \\
\hline $\begin{array}{ll}\text { Covered by } & \text { Inside } \\
\text { Covered by } & \text { Inside }\end{array}$ & $\begin{array}{l}{\left[\begin{array}{ll}\text { Covered by } & \text { Inside } \\
\text { Covers } & \text { Inside }\end{array}\right.}\end{array}$ & 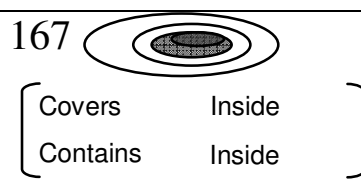 & $\begin{array}{l}168 \\
{\left[\begin{array}{ll}\text { Covers } & \text { Inside } \\
\text { Covers } & \text { Inside }\end{array}\right.}\end{array}$ \\
\hline$\left[\begin{array}{ll}\text { Equal } & \text { Inside } \\
\text { Equal } & \text { Inside }\end{array}\right.$ & {$\left[\begin{array}{ll}\text { Covered by } & \text { Inside } \\
\text { Equal } & \text { Inside }\end{array}\right.$} & $\begin{array}{lll}\substack{\text { Equal } \\
\text { Covers }} & \begin{array}{l}\text { Inside } \\
\text { Inside }\end{array} \\
171 & & \\
\end{array}$ & $\begin{array}{ll}\text { Overlap } & \text { Inside } \\
\text { Overlap } & \text { Inside }\end{array}$ \\
\hline 173 Inside & $\begin{array}{ll}\text { Disjoint } & \text { Disjoint } \\
\text { Disjoint } & \text { Meet }\end{array}$ & $\begin{array}{l}\text { Disjoint } \\
\text { Disjoint }\end{array}$ & 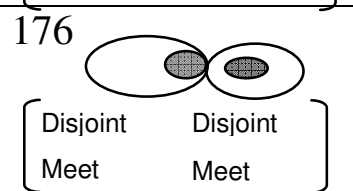 \\
\hline$\left[\begin{array}{l}\text { Meet } \\
\text { Meet }\end{array}\right.$ & {$\left[\begin{array}{ll}\text { Contains } & \text { Inside } \\
\text { Contains } & \text { Overlap }\end{array}\right.$} & {$\left[\begin{array}{ll}\text { Contains } & \text { Overlap } \\
\text { Contains } & \text { Overlap }\end{array}\right.$} & 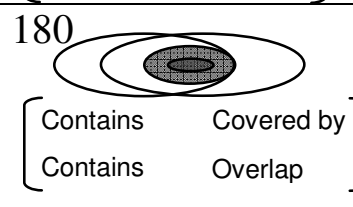 \\
\hline
\end{tabular}




\begin{tabular}{|c|c|c|c|}
\hline 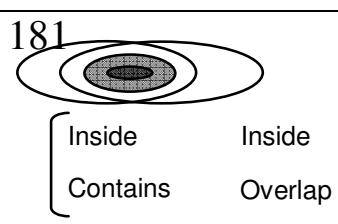 & {$\left[\begin{array}{ll}\text { Inside } & \text { Inside } \\
\text { Overlap } & \text { Overlap }\end{array}\right]$} & llllap & $\begin{array}{ll}{\left[\begin{array}{ll}\text { Meet } & \text { Inside } \\
\text { Contains } & \text { Overlap }\end{array}\right.} \\
184\end{array}$ \\
\hline $\begin{array}{ll}\text { Meet } & \text { Inside } \\
\text { Covers } & \text { Overlap }\end{array}$ & $\begin{array}{ll}\text { Meet } & \text { Covered by } \\
\text { Contains } & \text { Overlap }\end{array}$ & {$\left[\begin{array}{ll}\text { Meet } & \text { Covered by } \\
\text { Covers } & \text { Overlap }\end{array}\right]$} & $\begin{array}{ll}\text { Meet } & \text { Inside } \\
\text { Overlap } & \text { Overlap }\end{array}$ \\
\hline 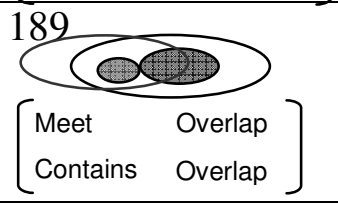 & {$\left[\begin{array}{ll}\text { Meet } & \text { Overlap } \\
\text { Overlap } & \text { Overlap }\end{array}\right.$} & {$\left[\begin{array}{ll}\begin{array}{l}\text { Meet } \\
\text { Overlap }\end{array} & \begin{array}{l}\text { Covered by } \\
\text { Overlap }\end{array}\end{array}\right]$} & $\begin{array}{ll}\text { Meet } & \text { Overlap } \\
\text { Covers } & \text { Overlap }\end{array}$ \\
\hline$\frac{\left[\begin{array}{ll}\text { Disjoint } & \text { Inside } \\
\text { Contains } & \text { Overlap }\end{array}\right]}{193000}$ & $\underbrace{194}_{\begin{array}{ll}\text { Disjoint } & \text { Inside } \\
\text { Covers } & \text { Overlap }\end{array}}$ & $\left.\begin{array}{ll}\text { Disjoint } & \text { Covered by } \\
\text { Contains } & \text { Overlap }\end{array}\right]$ & {$\left[\begin{array}{ll}\text { Disjoint } & \begin{array}{l}\text { Covered by } \\
\text { Covers }\end{array} \\
\text { Overlap }\end{array}\right]$} \\
\hline$\left[\begin{array}{ll}\text { Disjoint } & \text { Inside } \\
\text { Overlap } & \text { Overlap }\end{array}\right.$ & {$\left[\begin{array}{ll}\text { Disjoint } & \text { Overlap } \\
\text { Contains } & \text { Overlap }\end{array}\right.$} & {$\left[\begin{array}{ll}\text { Disjoint } & \text { Overlap } \\
\text { Overlap } & \text { Overlap }\end{array}\right.$} & {$\left[\begin{array}{ll}\text { Disjoint } & \text { Covered by } \\
\text { Overlap } & \text { Overlap }\end{array}\right]$} \\
\hline$\left[\begin{array}{ll}\text { Disjoint } & \text { Overlap } \\
\text { Covers } & \text { Overlap }\end{array}\right.$ & {$\left[\begin{array}{ll}\text { Disjoint } & \text { Disjoint } \\
\text { Overlap } & \text { Overlap }\end{array}\right.$} & {$\left[\begin{array}{ll}\text { Disjoint } & \text { Overlap } \\
\text { Disjoint } & \text { Overlap }\end{array}\right.$} & $\begin{array}{ll}\text { Disjoint } & \text { Meet } \\
\text { Overlap } & \text { Overlap }\end{array}$ \\
\hline $\begin{array}{ll}\text { Disjoint } & \text { Overlap } \\
\text { Meet } & \text { Overlap }\end{array}$ & 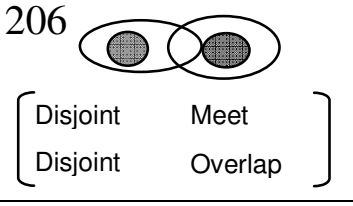 & {$\left[\begin{array}{ll}\text { Disjoint } & \text { Disjoint } \\
\text { Meet } & \text { Overlap }\end{array}\right.$} & $\begin{array}{ll}\text { Disjoint } & \text { Meet } \\
\text { Meet } & \text { Overlap }\end{array}$ \\
\hline $\begin{array}{ll}\text { Disjoint } & \text { Inside } \\
\text { Disjoint } & \text { Overlap }\end{array}$ & $\begin{array}{ll}\text { Disjoint } & \text { Disjoint } \\
\text { Contains } & \text { Overlap }\end{array}$ & $\begin{array}{l}\text { Disjoint } \\
\text { Overlap } \\
\end{array}$ & $\begin{array}{ll}{\left[\begin{array}{ll}\text { Disioint } \\
\text { Disjoint }\end{array}\right.} & \left.\begin{array}{l}\text { Covered by } \\
\text { Overlap }\end{array}\right]\end{array}$ \\
\hline $\begin{array}{ll}\text { Disjoint } & \text { Meet } \\
\text { Contains } & \text { Overlap }\end{array}$ & $\begin{array}{ll}\text { Disjoint } & \text { Inside } \\
\text { Meet } & \text { Overlap }\end{array}$ & {$\left[\begin{array}{ll}\text { Disjoint } & \text { Meet } \\
\text { Covers } & \text { Overlap }\end{array}\right.$} & $\begin{array}{ll}\text { Disjoint } & \text { Covered by } \\
\text { Meet } & \text { Overlap }\end{array}$ \\
\hline $\begin{array}{ll}\text { Disjoint } & \text { Disjoint } \\
\text { Disjoint } & \text { Overlap }\end{array}$ & $\begin{array}{ll}{\left[\begin{array}{ll}\text { Covers } & \text { Inside } \\
\text { Contains } & \text { Overlap }\end{array}\right.} \\
\end{array}$ & $\left.\begin{array}{ll}\text { Covers } & \text { Covered by } \\
\text { Contains } & \text { Overlap }\end{array}\right]$ & {$\left[\begin{array}{ll}\text { Covers } & \text { Covered by } \\
\text { Covers } & \text { Overlap }\end{array}\right]$} \\
\hline$\left[\begin{array}{ll}\text { Covers } & \text { Overlap } \\
\text { Contains } & \text { Overlap }\end{array}\right]$ & $\begin{array}{ll}\text { Covers } & \text { Overlap } \\
\text { Covers } & \text { Overlap }\end{array}$ & 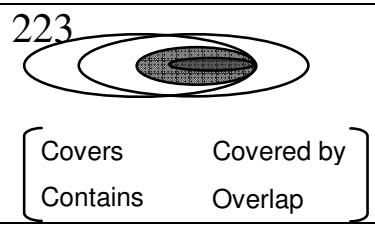 & {$\left[\begin{array}{ll}\text { Covered by } & \text { Covered by } \\
\text { Overlap } & \text { Overlap }\end{array}\right]$} \\
\hline
\end{tabular}




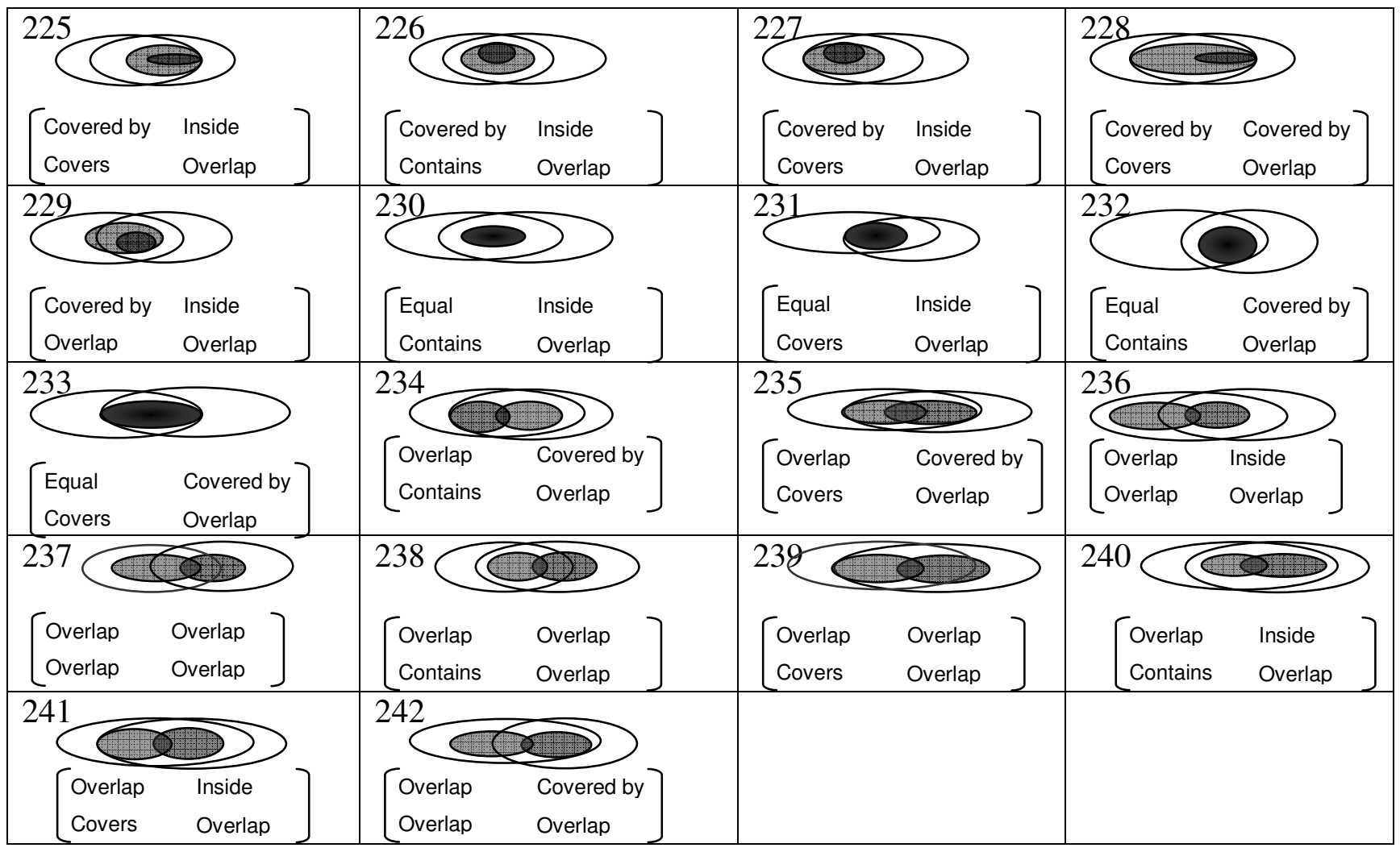

Figure 24. 242 topological relations between fuzzy regions

\section{Rules}

Table 4. Required rules for topological relations between fuzzy regions

Rule 1: Let $\widetilde{A}$ and $\widetilde{B}$ are two simple fuzzy regions, if Disjoint $\left(\tilde{A}_{\max }, \widetilde{B}_{\max }\right)$ then $\operatorname{Disjoint}\left(\tilde{A}_{\min }, \widetilde{B}_{\min }\right)$.

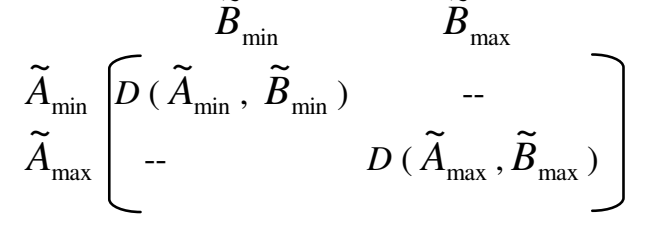

Proof: Let $\widetilde{A}$ and $\widetilde{B}$ two simple fuzzy regions where Disjoint $\left(\widetilde{A}_{\max }, \widetilde{B}_{\max }\right)$. Now, we suppose that Disjoint ( $\widetilde{A}_{\min }$, $\left.\widetilde{B}_{\min }\right) \neq$ Disjoint. In this case, the relation between minimal extent $\tilde{A}_{\min }$ and maximal extent $\tilde{A}_{\max }$ of a fuzzy region $\widetilde{A}$ or that between $\widetilde{B}_{\max }$ and $\widetilde{B}_{\min }$ does not correspond to Contains, Covers, Equal. Thus, there is a contradiction with definition 1.

Rule 2: Let $\widetilde{A}$ and $\widetilde{B}$ two fuzzy regions, if Meet $\quad\left(\tilde{A}_{\max }, \tilde{B}_{\max }\right)$ then

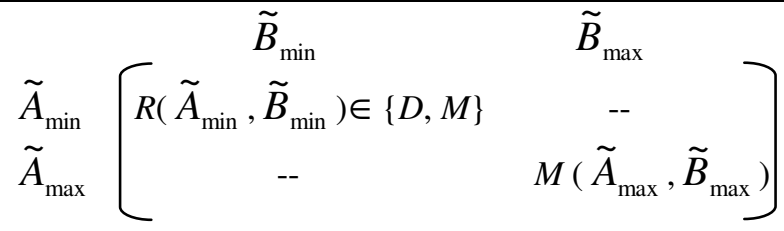

Proof: Let $\tilde{A}$ and $\widetilde{B}$ two simple fuzzy regions where Meet $\left(\widetilde{A}_{\max }, \widetilde{B}_{\max }\right)$. Now, we suppose that $R\left(\widetilde{A}_{\min }, \widetilde{B}_{\min }\right)$ $\notin\{$ Disjoint, Meet $\}$. In this case, relation between minimal extent $\tilde{A}_{\min }$ and maximal extent $\tilde{A}_{\max }, R$, $\left(\tilde{A}_{\max }, \tilde{A}_{\min }\right)$ or that between $\tilde{B}_{\max }$ and $\tilde{B}_{\min }, R^{\prime \prime}\left(\tilde{B}_{\max }, \tilde{B}_{\min }\right)$ does not correspond to Contains, Covers, Equal. Thus, there is a contradiction with definition 1. 


\begin{tabular}{|c|c|}
\hline $\begin{array}{l}\text { Sule 3: Let } \tilde{A} \text { and } \widetilde{B} \text { two fuzzy regions, if Contains } \\
\left(\tilde{A}_{\max }, \widetilde{B}_{\max }\right) \text { then Contains }\left(\tilde{A}_{\max }, \widetilde{B}_{\min }\right) \text { and vice } \\
\text { versa. }\end{array}$ & $\begin{array}{l}\text { ontains } \\
\text { ad vice }\end{array}$ \\
\hline \multicolumn{2}{|c|}{$\begin{array}{l}\text { roof: Let } \tilde{A} \text { and } \widetilde{B} \text { two simple fuzzy regions where Contains }\left(\tilde{A}_{\max }, \widetilde{B}_{\max }\right) \text {. According to definition } 1 \text {, any fuzzy } \\
\text { region } \tilde{A} \text { should respect the principal following condition: Equal }\left(\tilde{A}_{\max }, \tilde{A}_{\min }\right) \text {, Contains }\left(\tilde{A}_{\max }, \tilde{A}_{\min }\right) \text { or } \\
\text { Covers }\left(\tilde{A}_{\max }, \tilde{A}_{\min }\right) \text {. Moreover, Contains is a transitive topological relation according to Proposition 1. Then, } \\
\text { since Contains }\left(\tilde{A}_{\max }, \widetilde{B}_{\max }\right) \text { and } R\left(\tilde{B}_{\max }, \tilde{B}_{\min }\right)=\{\text { Contains, Covers, Equal }\} \text { then Contains }\left(\tilde{A}_{\max }, \tilde{B}_{\min }\right) \\
\text { and vice versa. }\end{array}$} \\
\hline $\begin{array}{l}\text { ule 4: Let } \tilde{A} \text { and } \widetilde{B} \text { two fuzzy regions, if Covers } \\
\\
\quad\left(\tilde{A}_{\max }, \widetilde{B}_{\max }\right) \text { then } R \quad\left(\tilde{A}_{\max }, \widetilde{B}_{\min }\right) \in \\
\\
\{\text { Contains, Covers }\} \text { and vice versa. }\end{array}$ & $\begin{array}{cc}\tilde{B}_{\min } & \tilde{B}_{\max } \\
-- & --\end{array}$ \\
\hline \multicolumn{2}{|c|}{$\begin{array}{l}\text { roof: Let } \tilde{A} \text { and } \tilde{B} \text { two simple fuzzy regions where Covers }\left(\tilde{A}_{\max }, \widetilde{B}_{\max }\right) \text {. According to definition } 1 \text {, any fuzzy } \\
\text { region } \tilde{A} \text { should respect the principal following condition: Equal }\left(\tilde{A}_{\max }, \tilde{A}_{\min }\right) \text {, Contains }\left(\tilde{A}_{\max }, \widetilde{A}_{\min }\right) \text { or } \\
\text { Covers }\left(\tilde{A}_{\max }, \tilde{A}_{\min }\right) \text {. Moreover, Covers is a transitive topological relation according to Proposition 1. By } \\
\text { considering Proposition } 2 \text {, if Contains }\left(\tilde{B}_{\max }, \tilde{B}_{\min }\right) \text { then Contains }\left(\tilde{A}_{\max }, \tilde{B}_{\min }\right) \text { else if } R \\
\left(\tilde{B}_{\max }, \tilde{B}_{\min }\right) \in\{\text { Covers, Equal }\} \text { then Covers }\left(\tilde{A}_{\max }, \tilde{B}_{\min }\right) \text { and vice versa. }\end{array}$} \\
\hline $\begin{array}{l}\text { Rule 5: Let } \tilde{A} \text { and } \widetilde{B} \text { two fuzzy regions, if Equal } \\
\left(\widetilde{A}_{\max }, \widetilde{B}_{\max }\right) \text { then } R \quad\left(\widetilde{A}_{\max }, \widetilde{B}_{\min }\right) \in \\
\\
\{\text { Contains, Covers, Equal }\} \text { and vice versa. }\end{array}$ & $\tilde{A}_{\min } \mid R\left(\tilde{A}_{\max }, \tilde{B}_{\min }\right.$ \\
\hline \multicolumn{2}{|c|}{ 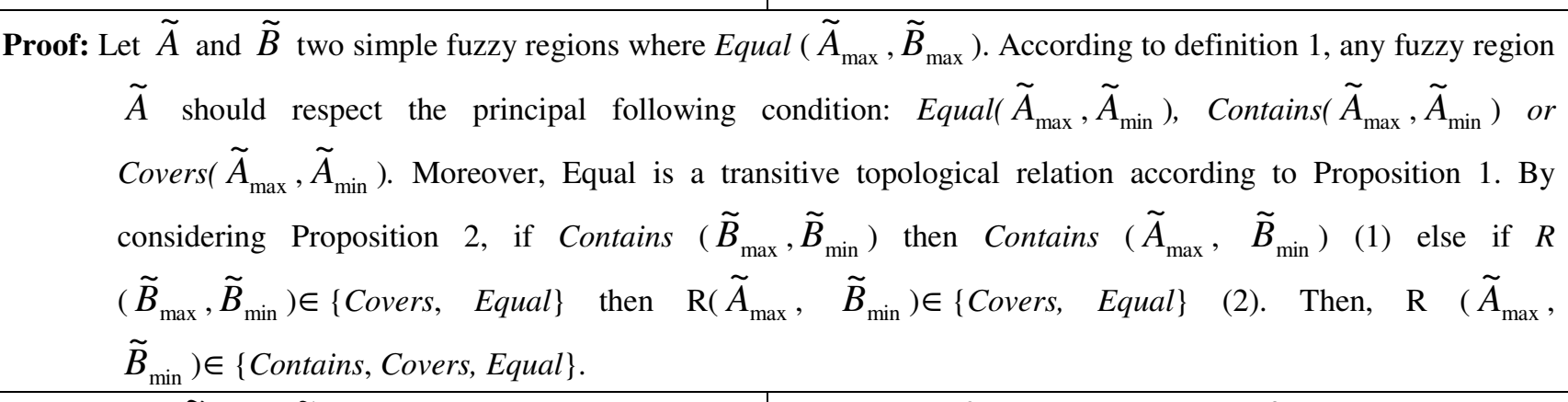 } \\
\hline $\begin{array}{l}\text { e 6: Let } \tilde{A} \text { and } \widetilde{B} \text { two fu } \\
\qquad \begin{array}{r}\left(\widetilde{A}_{\max }, \widetilde{B}_{\max }\right) \text { and Con } \\
R\left(\widetilde{A}_{\min }, \widetilde{B}_{\max }\right) \notin\{M e e\end{array}\end{array}$ & $\tilde{B}_{\min } \quad \widetilde{B}_{\max }$ \\
\hline & \\
\hline
\end{tabular}




\begin{tabular}{|c|c|c|}
\hline \multicolumn{3}{|c|}{$\begin{array}{l}\left.\widetilde{B}_{\min }\right) \text { and (1) then } R\left(\widetilde{B}_{\max }, \widetilde{B}_{\min }\right) \neq\{\text { Contains, Covers, Equal }\} . \text { Thus, there is a contradiction with } \\
\text { definition 1. }\end{array}$} \\
\hline \multicolumn{2}{|c|}{$\begin{array}{l}\text { Rule 7: Let } \tilde{A} \text { and } \widetilde{B} \text { two fuzzy regions, if Contains } \\
\left(\tilde{A}_{\max }, \widetilde{B}_{\max }\right) \text { and } \operatorname{Inside}\left(\tilde{A}_{\min }, \widetilde{B}_{\min }\right) \text { then } \\
\text { Inside }\left(\tilde{A}_{\min }, \widetilde{B}_{\max }\right) \text { and vice versa. }\end{array}$} & $\left.\begin{array}{cc}\widetilde{B}_{\min } & \tilde{B}_{\max } \\
I\left(\tilde{A}_{\min }, \tilde{B}_{\min }\right) & I\left(\tilde{A}_{\min }, \tilde{B}_{\max }\right) \\
-- & C\left(\tilde{A}_{\max }, \tilde{B}_{\max }\right)\end{array}\right]$ \\
\hline \multicolumn{3}{|c|}{$\begin{array}{l}\text { Proof: Let } \widetilde{A} \text { and } \widetilde{B} \text { two simple fuzzy regions where Contains }\left(\widetilde{A}_{\max }, \widetilde{B}_{\max }\right) \text { and Inside }\left(\widetilde{A}_{\min }, \widetilde{B}_{\min }\right) \text {. We suppose } \\
\text { now that } R\left(\widetilde{A}_{\min }, \widetilde{B}_{\max }\right) \notin\{\text { Inside }\} \text { (1). By considering definition } 1 \text { and proposition 2, since } R \\
\left(\widetilde{B}_{\max }, \widetilde{B}_{\min }\right) \in\{\text { Contains, Covers, Equal }\} \text { and Inside }\left(\widetilde{A}_{\min }, \widetilde{B}_{\min }\right) \text { then Inside }\left(\widetilde{A}_{\min }, \widetilde{B}_{\max }\right) \text { (2). Thus, there } \\
\text { is contradiction among (1) and (2). }\end{array}$} \\
\hline \multicolumn{2}{|c|}{$\begin{array}{l}\text { Rule 8: Let } \tilde{A} \text { and } \widetilde{B} \text { two simple fuzzy regions, if } \\
\text { Contains }\left(\widetilde{A}_{\max }, \widetilde{B}_{\max }\right) \text { and } \operatorname{Meet}\left(\widetilde{A}_{\min }, \widetilde{B}_{\min }\right) \\
\text { then } R \quad\left(\widetilde{A}_{\min }, \widetilde{B}_{\max }\right) \notin\{\text { Contains, Equal, } \\
\text { Covers, Disjoint }\} \text { and vice versa. }\end{array}$} & 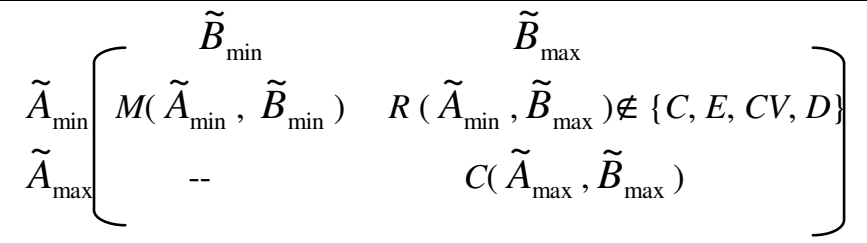 \\
\hline \multicolumn{3}{|c|}{ 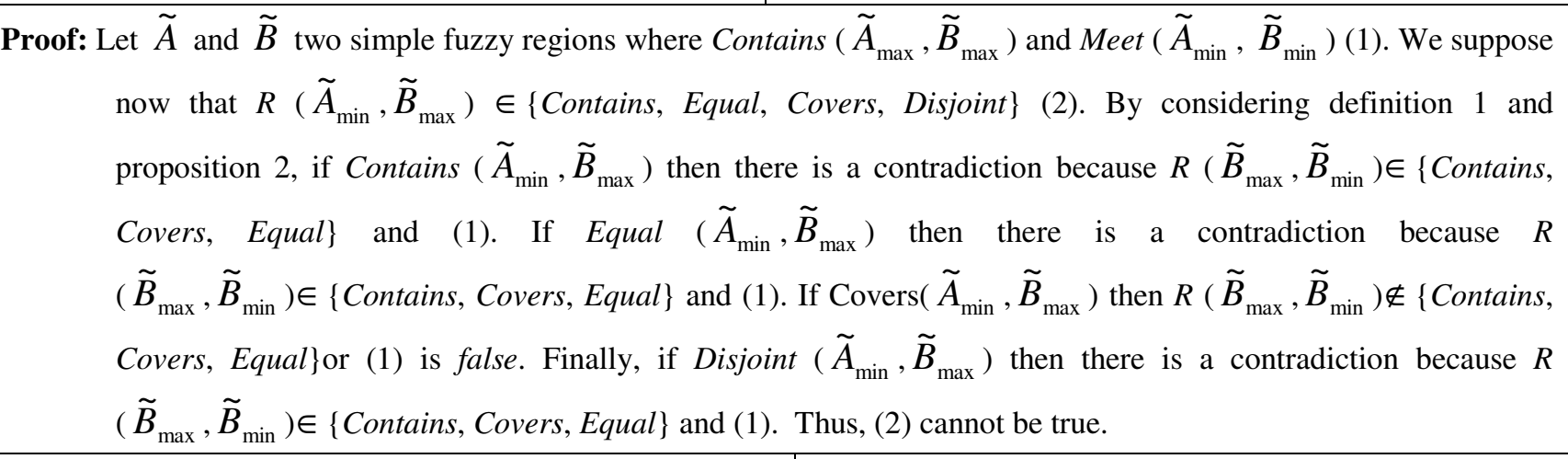 } \\
\hline \multicolumn{2}{|c|}{$\begin{aligned} \text { Rule 9: Let } \tilde{A} \text { and } \widetilde{B} \text { two fuzzy regions, if Contains } \\
\\
\left(\tilde{A}_{\max }, \widetilde{B}_{\max }\right) \text { and Covers }\left(\tilde{A}_{\min }, \widetilde{B}_{\min }\right) \text { then } R \\
\left(\tilde{A}_{\min }, \tilde{B}_{\max }\right) \notin\{\text { Meet, Disjoint }\} \text { and vice versa. }\end{aligned}$} & 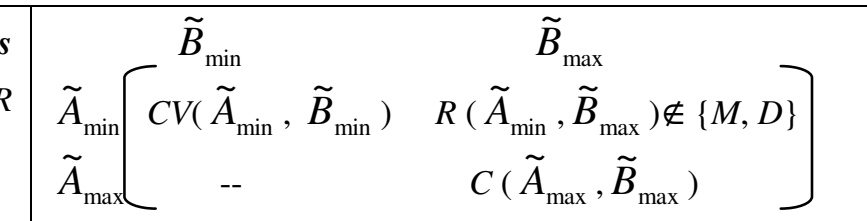 \\
\hline \multicolumn{3}{|c|}{ 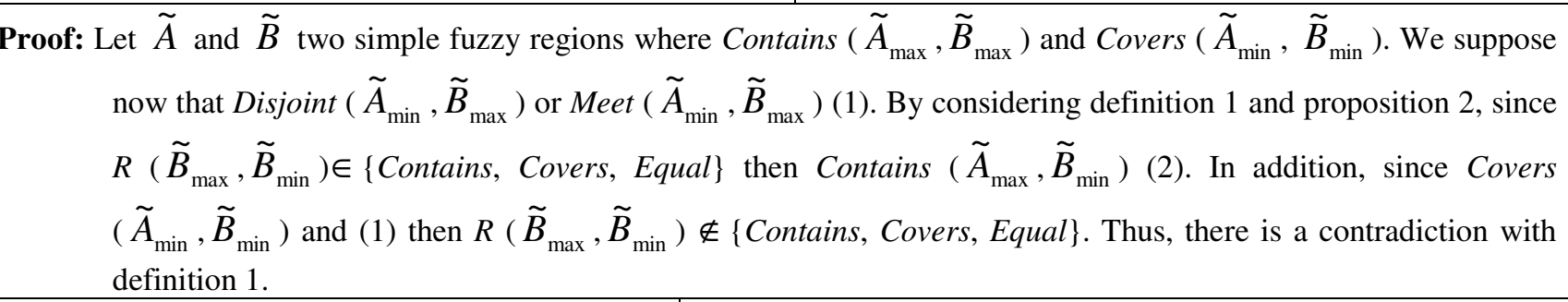 } \\
\hline 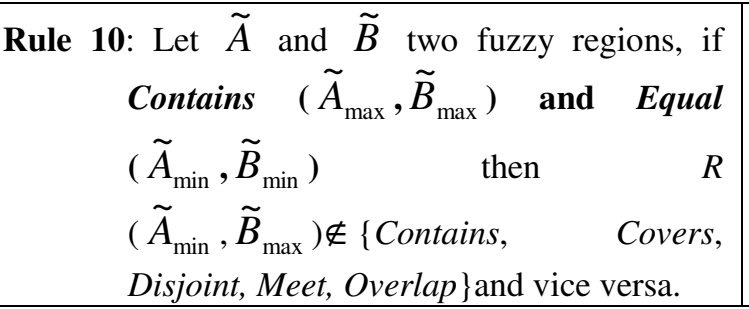 & & {$\left[\begin{array}{cc}E\left(\tilde{A}_{\text {min }}, \widetilde{B}_{\text {min }}\right) & \widetilde{B}_{\text {max }} \\
-- & C\left(\tilde{A}_{\min }, \widetilde{B}_{\max }\right) \notin\{C, C V, D, M, O\} \\
\left.\tilde{A}_{\max }, \tilde{B}_{\max }\right)\end{array}\right.$} \\
\hline & & \\
\hline
\end{tabular}




\begin{tabular}{|c|c|c|}
\hline \multicolumn{3}{|c|}{$\begin{array}{l}\left(\widetilde{B}_{\max }, \widetilde{B}_{\min }\right) \notin\{\text { Contains, Covers, Equal }\} \text { or }(1) \text { is false. By considering definition } 1 \text { and (1), there is a } \\
\text { contradiction and (2) cannot be true. }\end{array}$} \\
\hline \multicolumn{2}{|l|}{$\begin{array}{l}\text { Rule 11: Let } \tilde{A} \text { and } \widetilde{B} \text { two fuzzy regions, if Contains } \\
\left(\tilde{A}_{\max }, \widetilde{B}_{\max }\right) \text { and Covered by }\left(\tilde{A}_{\min }, \widetilde{B}_{\min }\right) \\
\text { then } R\left(\tilde{A}_{\min }, \widetilde{B}_{\max }\right) \in\{\text { Covered by, Inside }\} \text { and } \\
\text { vice versa. }\end{array}$} & $\begin{array}{cc}\tilde{B}_{\min } & \tilde{B}_{\max } \\
E\left(\tilde{A}_{\min }, \tilde{B}_{\min }\right) & R\left(\tilde{A}_{\min }, \tilde{B}_{\max }\right) \in\{C V B, I\} \\
-- & C\left(\tilde{A}_{\max }, \tilde{B}_{\max }\right)\end{array}$ \\
\hline \multicolumn{3}{|c|}{$\begin{array}{l}\text { Proof: Let } \tilde{A} \text { and } \widetilde{B} \text { two simple fuzzy regions where Contains }\left(\tilde{A}_{\max }, \widetilde{B}_{\max }\right) \text { and Covered by }\left(\tilde{A}_{\min }, \widetilde{B}_{\min }\right)(1) \text {. We } \\
\text { suppose now that } R\left(\tilde{A}_{\min }, \widetilde{B}_{\max }\right) \notin\{\text { Covered by, Inside }\} \text { then } R\left(\tilde{A}_{\min }, \widetilde{B}_{\max }\right) \in\{\text { Contains, Covers, Disjoint, } \\
\text { Meet, Overlap, Equal }\} \text { (2). If (2) then } R\left(\widetilde{B}_{\max }, \widetilde{B}_{\min }\right) \notin\{\text { Contains, Covers, Equal }\} \text { or (1) is false. By } \\
\text { considering definition } 1 \text { and (1), there is a contradiction and (2) cannot be true. }\end{array}$} \\
\hline $\begin{array}{l}\text { Rule 12: Let } \tilde{A} \text { and } \widetilde{B} \text { two fuzzy regions, if } \\
\text { Contains }\left(\tilde{A}_{\max }, \widetilde{B}_{\max }\right) \text { and Overlap }\left(\tilde{A}_{\min },\right. \\
\left.\tilde{B}_{\min }\right) \text { then } R\left(\tilde{A}_{\min }, \tilde{B}_{\max }\right) \in\{\text { Covered } \\
\text { by, Inside, Overlap }\} \text { and vice versa. }\end{array}$ & & 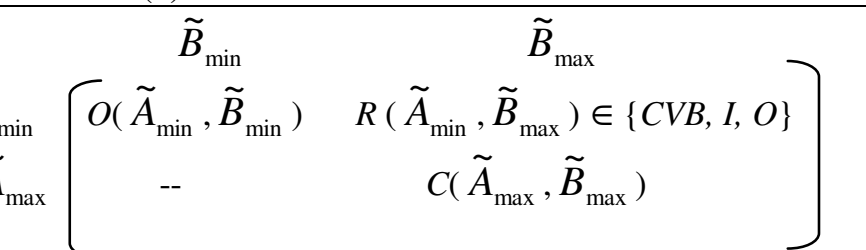 \\
\hline \multicolumn{3}{|c|}{$\begin{array}{l}\text { Proof: Let } \tilde{A} \text { and } \widetilde{B} \text { two simple fuzzy regions where Contains }\left(\tilde{A}_{\max }, \widetilde{B}_{\max }\right) \text { and Overlap }\left(\tilde{A}_{\min }, \widetilde{B}_{\min }\right)(1) \text { We } \\
\text { suppose now that } R\left(\tilde{A}_{\min }, \widetilde{B}_{\max }\right) \in\{\text { Contains, Covers, Disjoint, Meet, Equal }\} \text { (2). If (2) then } R \\
\left(\widetilde{B}_{\max }, \widetilde{B}_{\min }\right) \notin\{\text { Contains, Covers, Equal }\} \text { or (1) is false. By considering definition } 1 \text { and (1), there is a } \\
\text { contradiction and (2) cannot be true. }\end{array}$} \\
\hline \multicolumn{2}{|l|}{$\begin{array}{l}\text { Rule 13: Let } \tilde{A} \text { and } \widetilde{B} \text { two fuzzy regions, if Covers } \\
\left(\tilde{A}_{\max }, \widetilde{B}_{\max }\right) \text { and Contains }\left(\tilde{A}_{\min }, \widetilde{B}_{\min }\right) \\
\text { then } R\left(\tilde{A}_{\min }, \widetilde{B}_{\max }\right) \notin\{\text { Disjoint, Meet }\} \text { and } \\
\text { vice versa. }\end{array}$} & $\begin{array}{c}\tilde{A}_{\min } \\
\tilde{A}_{\max }\end{array}\left[\begin{array}{c}\tilde{B}_{\min } \\
C\left(\tilde{A}_{\min }, \tilde{B}_{\min }\right) \\
--\end{array}\right.$ \\
\hline \multicolumn{3}{|c|}{$\begin{array}{l}\text { Proof: Let } \tilde{A} \text { and } \widetilde{B} \text { two simple fuzzy regions where Covers }\left(\widetilde{A}_{\max }, \widetilde{B}_{\max }\right) \text { and Contains }\left(\tilde{A}_{\min }, \widetilde{B}_{\min }\right)(1) \text {. We } \\
\text { suppose now that Disjoint }\left(\tilde{A}_{\min }, \widetilde{B}_{\max }\right) \text { or Meet }\left(\tilde{A}_{\min }, \widetilde{B}_{\max }\right)(2) \text {. If (2) then } R\left(\widetilde{B}_{\max }, \widetilde{B}_{\min }\right) \notin\{\text { Contains, } \\
\text { Covers, Equal }\} \text { or (1) is false. By considering definition } 1 \text { and (1), there is a contradiction and (2) cannot be } \\
\text { true. }\end{array}$} \\
\hline \multicolumn{2}{|l|}{$\begin{array}{l}\text { Rule 14: Let } \tilde{A} \text { and } \widetilde{B} \text { two fuzzy regions, if Covers } \\
\left(\tilde{A}_{\max }, \widetilde{B}_{\max }\right) \text { and } \operatorname{Inside}\left(\tilde{A}_{\min }, \widetilde{B}_{\min }\right) \text { then } \\
\text { Inside }\left(\tilde{A}_{\min }, \widetilde{B}_{\max }\right) \text { and vice versa. }\end{array}$} & $\begin{array}{c}\tilde{A}_{\min } \\
\tilde{A}_{\max }\end{array}\left[\begin{array}{cc}I\left(\tilde{A}_{\min }, \tilde{B}_{\min }\right) & I\left(\tilde{A}_{\min }, \widetilde{B}_{\max }\right) \\
-- & C V\left(\tilde{A}_{\max }, \widetilde{B}_{\max }\right)\end{array}\right]$ \\
\hline \multicolumn{3}{|c|}{ 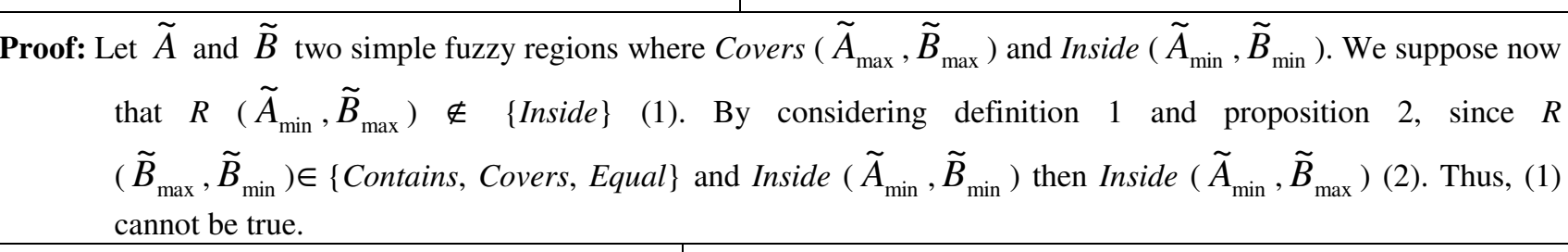 } \\
\hline $\begin{array}{l}\text { ule 15: Let } \tilde{A} \text { and } \widetilde{B} \text { two simple fuzzy regions, } \\
\text { if Covers }\left(\widetilde{A}_{\max }, \widetilde{B}_{\max }\right) \text { and } \boldsymbol{R}\left(\widetilde{A}_{\min },\right. \\
\left.\widetilde{B}_{\min }\right) \in\{\text { Disjoint, Meet }\} \text { then } R \\
\left(\widetilde{A}_{\min }, \widetilde{B}_{\max }\right) \notin\{\text { Contains, } \\
\text { Disjoint, Equal }\} \text { and vice versa. }\end{array}$ & & $\tilde{B}_{\min } \quad \tilde{B}_{\max }$ \\
\hline
\end{tabular}


Proof: Let $\tilde{A}$ and $\widetilde{B}$ two simple fuzzy regions where Covers $\left(\tilde{A}_{\max }, \widetilde{B}_{\max }\right)$ and $\{$ Meet, Disjoint $\}\left(\tilde{A}_{\min }, \widetilde{B}_{\min }\right)$ (1). We suppose now that $R\left(\tilde{A}_{\min }, \tilde{B}_{\max }\right) \in\{$ Contains, Covers, Disjoint, Equal $\}$ (2). If (2) then $R$ $\left(\widetilde{B}_{\max }, \widetilde{B}_{\min }\right) \notin\{$ Contains, Covers, Equal $\}$ or (1) is false. By considering definition 1 and (1), there is a contradiction and (2) cannot be true.

Rule 16: Let $\tilde{A}$ and $\widetilde{B}$ two simple fuzzy regions, if Covers $\left(\tilde{A}_{\max }, \widetilde{B}_{\max }\right)$ and $\boldsymbol{R}\left(\tilde{A}_{\min }, \widetilde{B}_{\min }\right) \in\{$ Equal, Covered by $\}$ then $R\left(\tilde{A}_{\min }, \tilde{B}_{\max }\right) \in\{$ Covered by, Inside $\}$ and vice versa.

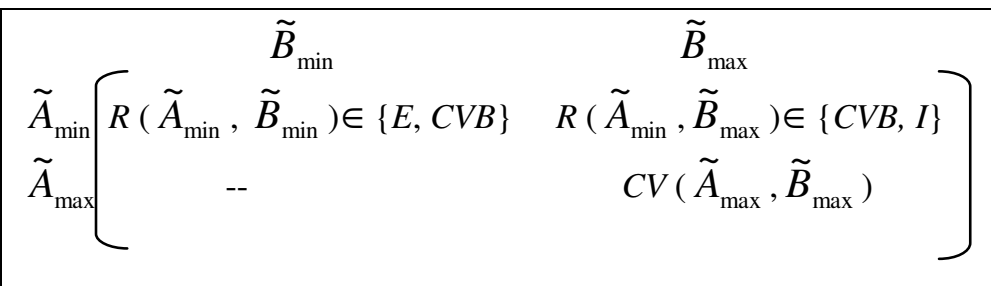

Proof: Let $\tilde{A}$ and $\widetilde{B}$ two simple fuzzy regions where $\operatorname{Covers}\left(\tilde{A}_{\max }, \widetilde{B}_{\max }\right)$ and $R\left(\tilde{A}_{\min }, \widetilde{B}_{\max }\right) \in\{$ Covered by, Inside $\}$ (1). We suppose now that $R\left(\tilde{A}_{\min }, \widetilde{B}_{\max }\right) \notin\{$ Covered by, Inside $\}$ (2). If (2) then $R$ $\left(\widetilde{B}_{\max }, \widetilde{B}_{\min }\right) \notin\{$ Contains, Covers, Equal $\}$ or (1) is false. By considering definition 1 and (1), there is a contradiction and (2) cannot be true.

Rule 17: Let $\tilde{A}$ and $\widetilde{B}$ two simple fuzzy regions, if Covers $\left(\tilde{A}_{\max }, \widetilde{B}_{\max }\right)$ and Overlap $\left(\tilde{A}_{\min }\right.$, $\left.\tilde{B}_{\min }\right)$ then $R\left(\tilde{A}_{\min }, \widetilde{B}_{\max }\right) \in\{$ Covered by, Inside, Overlap $\}$ and vice versa.

\section{$\widetilde{B}_{\min } \tilde{B}_{\max }$}

$\tilde{A}_{\min }\left[O\left(\tilde{A}_{\min }, \tilde{B}_{\text {min }}\right) \quad R\left(\tilde{A}_{\min }, \tilde{B}_{\text {max }}\right) \in\{C V B, I, O\}\right.$ $\tilde{A}_{\text {max }} \quad-\quad C V\left(\tilde{A}_{\text {max }}, \tilde{B}_{\text {max }}\right)$

Proof: Let $\tilde{A}$ and $\tilde{B}$ two simple fuzzy regions where Covers $\left(\tilde{A}_{\max }, \widetilde{B}_{\max }\right)$ and Overlap $\left(\tilde{A}_{\min }, \widetilde{B}_{\min }\right)(1)$. We suppose now that $R\left(\tilde{A}_{\min }, \widetilde{B}_{\max }\right) \notin\{$ Covered by, Inside, Overlap $\}$ (2). If (2) then $R\left(\widetilde{B}_{\max }, \widetilde{B}_{\min }\right) \notin\{$ Contains, Covers, Equal\} or (1) is false. By considering definition 1 and (1), there is a contradiction and (2) cannot be true.

Rule 18: Let $\tilde{A}$ and $\widetilde{B}$ two simple fuzzy regions, if Meet $\left(\tilde{A}_{\max }, \tilde{B}_{\max }\right)$ and Meet $\left(\tilde{A}_{\text {min }}, \tilde{B}_{\min }\right)$ then $\operatorname{Meet}\left(\tilde{A}_{\min }, \widetilde{B}_{\max }\right)$ and $\operatorname{Meet}\left(\tilde{A}_{\max }, \widetilde{B}_{\text {min }}\right)$ and vice versa.

\section{$\tilde{B}_{\min } \quad \tilde{B}_{\max }$}

$\tilde{A}_{\min }$
$\tilde{A}_{\max }$$\left[\begin{array}{cc}M\left(\tilde{A}_{\min }, \tilde{B}_{\min }\right) & M\left(\tilde{A}_{\min }, \tilde{B}_{\max }\right) \\ M\left(\tilde{A}_{\max }, \tilde{B}_{\min }\right) & M\left(\tilde{A}_{\max }, \tilde{B}_{\max }\right)\end{array}\right]$

Proof: Let $\tilde{A}$ and $\widetilde{B}$ two simple fuzzy regions where Meet $\left(\widetilde{A}_{\max }, \widetilde{B}_{\max }\right)$ and Meet $\left(\widetilde{A}_{\min }, \widetilde{B}_{\min }\right)(1)$. We suppose now that $R\left(\tilde{A}_{\min }, \widetilde{B}_{\max }\right) \neq$ Meet (2) and $R\left(\widetilde{A}_{\max }, \widetilde{B}_{\text {min }}\right) \neq$ Meet (3). If (2) then $R\left(\widetilde{B}_{\max }, \widetilde{B}_{\min }\right) \notin\{$ Contains, Covers, Equal\} or (1) is false. Thus, (2) cannot be true. In the same way, if (3) then there is a contradiction because $R\left(\widetilde{B}_{\max }, \widetilde{B}_{\text {min }}\right) \notin\{$ Contains, Covers, Equal $\}$ or (1) is false. By considering definition 1 and (1), there is a contradiction and (3) cannot be true.

Rule 19: Let $\tilde{A}$ and $\tilde{B}$ two simple fuzzy regions, if Meet $\left(\tilde{A}_{\max }, \widetilde{B}_{\max }\right)$ and Disjoint $\left(\widetilde{A}_{\min }, \widetilde{B}_{\min }\right)$ then $R\left(\widetilde{A}_{\min }, \widetilde{B}_{\max }\right) \in\{$ Meet, Disjoint $\}$ and $R\left(\tilde{A}_{\max }, \widetilde{B}_{\min }\right) \in\{$ Meet, Disjoint $\}$ and vice versa.

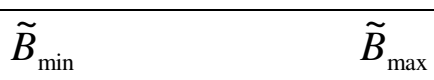

$$
\begin{aligned}
& \tilde{A}_{\min } \\
& \tilde{A}_{\max }
\end{aligned}\left[\begin{array}{cc}
D\left(\tilde{A}_{\text {min }}, \tilde{B}_{\min }\right) & R\left(\tilde{A}_{\min }, \tilde{B}_{\max }\right) \in\{M, D\} \\
R\left(\tilde{A}_{\max }, \tilde{B}_{\min }\right) \in\{M, D\} & M\left(\tilde{A}_{\max }, \tilde{B}_{\max }\right)
\end{array}\right]
$$

Proof: Let $\widetilde{A}$ and $\widetilde{B}$ two simple fuzzy regions where $\operatorname{Meet}\left(\tilde{A}_{\max }, \widetilde{B}_{\max }\right)$ and $\operatorname{Disjoint}\left(\tilde{A}_{\min }, \widetilde{B}_{\min }\right)(1)$. We suppose now that $R\left(\widetilde{A}_{\text {min }}, \widetilde{B}_{\text {max }}\right) \notin\{$ Meet, Disjoint $\}$ (2) and $R\left(\tilde{A}_{\text {max }}, \widetilde{B}_{\text {min }}\right) \notin\{$ Meet, Disjoint $\}$ (3). If (2) then there $R$ $\left(\widetilde{B}_{\max }, \widetilde{B}_{\min }\right) \notin\{$ Contains, Covers, Equal $\}$ or (1) is false. By considering definition 1 and (1), there is a 


\begin{tabular}{|c|c|}
\hline \multicolumn{2}{|c|}{$\begin{array}{l}\text { contradiction and (2) cannot be true. In the same way, if (3) then } R\left(\widetilde{B}_{\max }, \widetilde{B}_{\min }\right) \notin\{\text { Contains, Covers, Equal }\} \\
\text { or (1) is false. By considering definition } 1 \text { and (1), there is a contradiction and (3) cannot be true. }\end{array}$} \\
\hline $\begin{array}{l}\text { Rule 20 : Let } \tilde{A} \text { and } \widetilde{B} \text { two simple fuzzy regions, if } \\
\text { Overlap } \quad\left(\tilde{A}_{\max }, \widetilde{B}_{\max }\right) \text { then } R \\
\left(\tilde{A}_{\max }, \tilde{B}_{\min }\right) \notin\{\text { Equal, Inside, Covered by }\} \\
\text { and vice versa. }\end{array}$ & 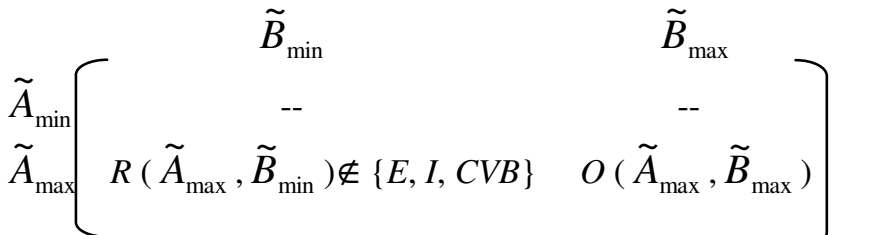 \\
\hline \multicolumn{2}{|c|}{$\begin{array}{l}\text { Proof: Let } \tilde{A} \text { and } \tilde{B} \text { two simple fuzzy regions where Overlap }\left(\tilde{A}_{\max }, \tilde{B}_{\max }\right) \text {. According to definition 1, any fuzzy } \\
\text { region } \tilde{A} \text { should respect the principal following condition: Equal }\left(\tilde{A}_{\max }, \tilde{A}_{\min }\right) \text {, Contains }\left(\tilde{A}_{\max }, \tilde{A}_{\min }\right) \text { or } \\
\text { Covers }\left(\tilde{A}_{\max }, \tilde{A}_{\min }\right) \text { (1). We suppose now that } R\left(\tilde{A}_{\max }, \tilde{B}_{\min }\right) \in\{\text { Equal, Inside, Covered by }\} \text { (2). By } \\
\text { considering definition } 1 \text { and proposition 2, if (1) and (2) then } R\left(\tilde{B}_{\max }, \tilde{B}_{\min }\right) \notin\{\text { Contains, Covers, Equal }\} \text {. } \\
\text { Thus, there is a contradiction with definition 1. }\end{array}$} \\
\hline $\begin{array}{l}\text { Rule 21: Let } \tilde{A} \text { and } \widetilde{B} \text { two simple fuzzy regions, if } \\
\text { Overlap }\left(\tilde{A}_{\max }, \widetilde{B}_{\max }\right) \text { and Contains }\left(\tilde{A}_{\min } \text {, }\right. \\
\left.\tilde{B}_{\min }\right) \text { then } R\left(\tilde{A}_{\min }, \widetilde{B}_{\max }\right) \in\{\text { Overlap, Inside, } \\
\text { Covered by }\} \text { and Contains }\left(\tilde{A}_{\max }, \tilde{B}_{\min }\right) \text { and } \\
\text { vice versa. }\end{array}$ & $\begin{array}{c}\tilde{A}_{\min } \\
\tilde{A}_{\max } \\
\tilde{A}_{\min }\end{array}\left[\begin{array}{cc}\mathrm{C}\left(\tilde{A}_{\min }, \tilde{B}_{\min }\right) & R\left(\tilde{A}_{\min }, \tilde{B}_{\max }\right) \in\{O, I, C V B\} \\
C\left(\tilde{A}_{\max }, \tilde{B}_{\min }\right) & O\left(\tilde{A}_{\max }, \tilde{B}_{\max }\right)\end{array}\right]$ \\
\hline \multicolumn{2}{|c|}{$\begin{array}{l}\text { Proof: Let } \tilde{A} \text { and } \widetilde{B} \text { two simple fuzzy regions where Overlap }\left(\tilde{A}_{\max }, \widetilde{B}_{\max }\right) \text { and Contains }\left(\tilde{A}_{\min }, \widetilde{B}_{\min }\right)(1) \text {. We } \\
\text { suppose now that } R\left(\widetilde{A}_{\min }, \widetilde{B}_{\max }\right) \notin\{\text { Overlap, Inside, Covered by }\} \text { (2) and } R\left(\widetilde{A}_{\max }, \widetilde{B}_{\min }\right) \neq \text { Contains (3). By } \\
\text { considering definition } 1 \text { and proposition } 2 \text {, if (2) then } R\left(\widetilde{B}_{\max }, \widetilde{B}_{\min }\right) \notin\{\text { Contains, Covers, Equal }\} \text { or (1) is } \\
\text { false. Thus, (2) cannot be true because there is a contradiction. In the same way, if (3) then } R \\
\left(\widetilde{B}_{\max }, \widetilde{B}_{\min }\right) \notin\{\text { Contains, Covers, Equal }\} \text { or (1) is false. By considering definition } 1 \text { and proposition } 2 \text {, (3) } \\
\text { cannot be true because there is also a contradiction. }\end{array}$} \\
\hline 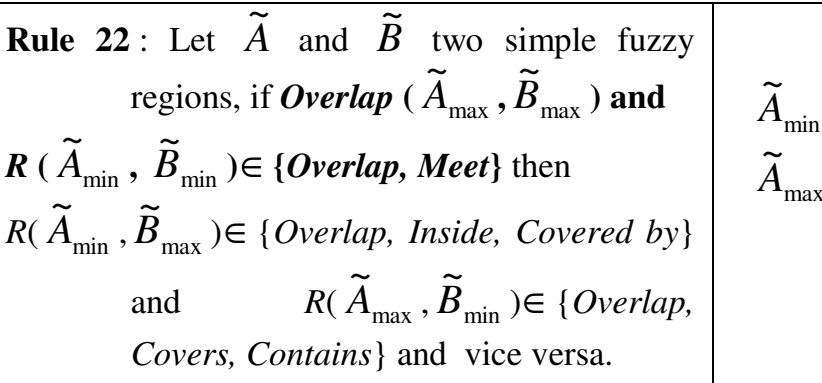 & {$\left[\begin{array}{ccc} & \tilde{B}_{\text {min }} & \tilde{B}_{\max } \\
R\left(\tilde{A}_{\min }, \tilde{B}_{\min }\right) \in\{O, M\} & R\left(\tilde{A}_{\min }, \tilde{B}_{\max }\right) \in\{O, I, C V B\} \\
R\left(\tilde{A}_{\max }, \tilde{B}_{\min }\right) \in\{O, C V, C\} & O\left(\tilde{A}_{\max }, \tilde{B}_{\max }\right)\end{array}\right]$} \\
\hline $\begin{array}{l}\text { of: Let } \tilde{A} \text { and } \widetilde{B} \text { two simple fuzzy regions where } O \\
\text { (1). We suppose now that } R\left(\tilde{A}_{\min }, \widetilde{B}_{\max }\right) \notin\{C \\
\text { Covers, Contains }\} \text { (3). If (2) then } R\left(\widetilde{B}_{\max }, \widetilde{B}_{\min }\right. \\
\text { definition } 1 \text { and proposition 2, there is a contrad } \\
\left(\widetilde{B}_{\max }, \widetilde{B}_{\min }\right) \notin\{\text { Contains, Covers, Equal }\} \text { or }(1 \\
\text { is contradiction and (3) cannot be true. }\end{array}$ & $\begin{array}{l}\text { lap }\left(\tilde{A}_{\max }, \widetilde{B}_{\max }\right) \text { and } R\left(\tilde{A}_{\min }, \widetilde{B}_{\min }\right) \in\{\text { Overlap, Meet }\} \\
\text { rlap, Inside, Covered by }\} \text { (2) } R\left(\tilde{A}_{\max }, \widetilde{B}_{\min }\right) \notin\{\text { Overlap, } \\
\{\text { Contains, Covers, Equal }\} \text { or (1) is false. By considering } \\
\text { ion and (2) cannot be true. In the same way, if (3) then } R \\
\text { false. By considering definition } 1 \text { and proposition } 2 \text {, there }\end{array}$ \\
\hline $\begin{array}{l}\text { Rule 23: Let } \tilde{A} \text { and } \widetilde{B} \text { two simple fuzzy regions, if } \\
\text { Overlap }\left(\tilde{A}_{\max }, \tilde{B}_{\max }\right) \text { and Equal }\left(\tilde{A}_{\min } \text {, }\right. \\
\left.\tilde{B}_{\min }\right) \text { then } \boldsymbol{R}\left(\tilde{A}_{\min }, \widetilde{B}_{\max }\right) \in\{\text { Overlap, Inside, } \\
\text { Covered by }\} \text { and } R\left(\tilde{A}_{\max }, \widetilde{B}_{\min }\right) \in\{\text { Overlap, } \\
\text { Covers, Contains }\} \text { and vice versa. }\end{array}$ & $\begin{array}{c}\tilde{B}_{\min } \\
\tilde{A}_{\min } \\
\tilde{A}_{\max }\end{array}\left[\begin{array}{cc}E\left(\tilde{A}_{\min }, \tilde{B}_{\min }\right) & R\left(\tilde{A}_{\max }, \tilde{B}_{\min }\right) \in\{I, C V B\} \\
R\left(\tilde{A}_{\max }, \tilde{B}_{\min }\right) \in\{C V, C\} & O\left(\tilde{A}_{\max }, \tilde{B}_{\max }\right)\end{array}\right]$ \\
\hline
\end{tabular}


Proof: Let $\tilde{A}$ and $\tilde{B}$ two simple fuzzy regions where Overlap $\left(\tilde{A}_{\max }, \widetilde{B}_{\max }\right)$ and Equal $\left(\tilde{A}_{\min }, \widetilde{B}_{\min }\right)$ (1). We suppose now that $R\left(\tilde{A}_{\min }, \widetilde{B}_{\max }\right) \notin\{$ Inside, Covered by $\}$ (2) $R\left(\widetilde{A}_{\text {max }}, \widetilde{B}_{\text {min }}\right) \notin\{$ Covers, Contains $\}$ (3). If (2) then $R$ $\left(\widetilde{B}_{\max }, \widetilde{B}_{\min }\right) \notin\{$ Contains, Covers, Equal $\}$ or (1) is false. By considering definition 1 and proposition 2, there is contradiction and (2) cannot be true. In the same way, if (3) then $R\left(\widetilde{B}_{\max }, \widetilde{B}_{\min }\right) \notin\{$ Contains, Covers, Equal\} or (1) is false. By considering definition 1 and proposition 2, (3) cannot be true because there is also a contradiction.

Rule 24: Let $\tilde{A}$ and $\widetilde{B}$ two simple fuzzy regions, if

Overlap $\left(\tilde{A}_{\max }, \widetilde{B}_{\max }\right)$ and Inside $\left(\tilde{A}_{\min }, \widetilde{B}_{\min }\right)$

then $R\left(\tilde{A}_{\min }, \widetilde{B}_{\max }\right) \in\{$ Inside $\}$ and

$\left(\tilde{A}_{\max }, \widetilde{B}_{\min }\right) \in\{$ Overlap, Covers, Contains $\}$ and

\begin{tabular}{|c|c|c|}
\hline & $\tilde{B}_{\min }$ & $\widetilde{B}_{\max }$ \\
\hline$A_{\mathrm{mi}}$ & $I\left(\tilde{A}_{\min }, \tilde{B}_{\min }\right)$ & $I\left(\tilde{A}_{\max }, \widetilde{B}_{\min }\right)$ \\
\hline$\tilde{\mu}$ & $R\left(\tilde{A}_{\max }, \tilde{B}_{\min }\right) \in\{C, C V, O\}$ & $O\left(\tilde{A}_{\max }, \tilde{B}_{\max }\right)$ \\
\hline
\end{tabular}
vice versa.

Proof: Let $\widetilde{A}$ and $\widetilde{B}$ two simple fuzzy regions where Overlap $\left(\widetilde{A}_{\max }, \widetilde{B}_{\max }\right)$ and Inside $\left(\widetilde{A}_{\text {min }}, \widetilde{B}_{\text {min }}\right)$ (1). We suppose now that $R\left(\tilde{A}_{\text {min }}, \widetilde{B}_{\text {max }}\right) \notin\{$ Inside $\}(2) R\left(\tilde{A}_{\text {max }}, \widetilde{B}_{\text {min }}\right) \notin\{$ Covers, Contains, Overlap $\}$ (3). If (2) then $R$ $\left(\widetilde{B}_{\max }, \widetilde{B}_{\min }\right) \notin\{$ Contains, Covers, Equal $\}$ or (1) is false. By considering definition 1 and proposition 2, there is a contradiction and (2) cannot be true. In the same way, if (3) then $R\left(\widetilde{B}_{\max }, \widetilde{B}_{\min }\right) \notin\{$ Contains, Covers, Equal $\}$ or (1) is false. By considering definition 1 and proposition 2, (3) cannot be true because there is also a contradiction.

Rule 25: Let $\tilde{A}$ and $\widetilde{B}$ two simple fuzzy regions, if Overlap $\left(\tilde{A}_{\max }, \widetilde{B}_{\max }\right)$ and Covers $\left(\tilde{A}_{\min }\right.$, $\left.\widetilde{B}_{\text {min }}\right)$ then $R\left(\tilde{A}_{\min }, \widetilde{B}_{\max }\right) \in\{$ Inside, Covered by, Overlap $\}$ and $R\left(\tilde{A}_{\max }, \tilde{B}_{\text {min }}\right) \in\{$ Covers, Contains $\} \quad$ and vice versa.

Proof: Let $\tilde{A}$ and $\tilde{B}$ two simple fuzzy regions where Overlap $\left(\tilde{A}_{\max }, \widetilde{B}_{\max }\right)$ and Covers $\left(\tilde{A}_{\min }, \widetilde{B}_{\min }\right)(1)$. We suppose now that $R\left(\widetilde{A}_{\min }, \widetilde{B}_{\max }\right) \notin\{$ Inside, Covered by, Overlap $\}$ (2) $R\left(\widetilde{A}_{\max }, \widetilde{B}_{\min }\right) \notin\{$ Covers, Contains $\}$ (3). If (2) then $R\left(\widetilde{B}_{\text {max }}, \widetilde{B}_{\text {min }}\right) \notin\{$ Contains, Covers, Equal $\}$ or (1) is false. By considering definition 1 and proposition 2, (2) cannot be true because there is a contradiction. In the same way, if (3) then $R$ $\left(\widetilde{B}_{\max }, \widetilde{B}_{\min }\right) \notin\{$ Contains, Covers, Equal $\}$ or (1) is false. By considering definition 1 and proposition 2, (3) cannot be true because there is also a contradiction.

Rule 26: Let $\tilde{A}$ and $\tilde{B}$ two simple fuzzy regions, if Overlap $\left(\tilde{A}_{\max }, \tilde{B}_{\max }\right)$ and Disjoint $\left(\tilde{A}_{\text {min }}, \tilde{B}_{\text {min }}\right)$ then $R\left(\tilde{A}_{\text {min }}, \tilde{B}_{\text {max }}\right) \notin\{$ Equal, Contains, Covers $\} \quad$ and

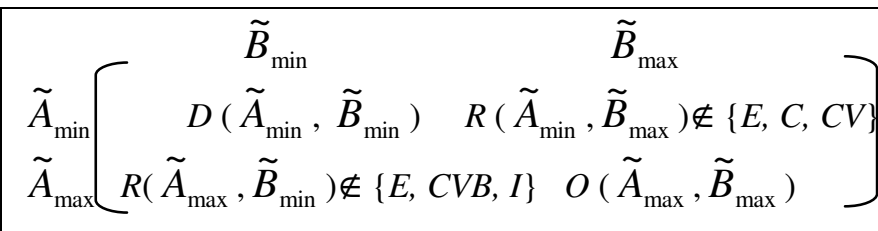
$R\left(\tilde{A}_{\max }, \widetilde{B}_{\min }\right) \notin\{$ Equal, Covered by, Inside $\}$ and vice versa.

Proof: Let $\tilde{A}$ and $\widetilde{B}$ two simple fuzzy regions where Overlap $\left(\widetilde{A}_{\max }, \widetilde{B}_{\max }\right)$ and Disjoint $\left(\widetilde{A}_{\min }, \widetilde{B}_{\min }\right)(1)$. We suppose now that $R\left(\tilde{A}_{\min }, \widetilde{B}_{\max }\right) \notin\{$ Equal, Contains, Covers $\}$ (2) $R\left(\tilde{A}_{\max }, \widetilde{B}_{\min }\right) \notin\{$ Equal, Covered by, Inside $\}$ (3). If (2) then $R\left(\widetilde{B}_{\text {max }}, \widetilde{B}_{\text {min }}\right) \notin\{$ Contains, Covers, Equal $\}$ or (1) is false. By considering definition 1 and proposition 2, (2) cannot be true because there is a contradiction. In the same way, if (3) then $R$ $\left(\widetilde{B}_{\max }, \widetilde{B}_{\min }\right) \notin\{$ Contains, Covers, Equal $\}$ or (1) is false. By considering definition 1 and proposition 2, (3) 


\begin{tabular}{|c|c|}
\hline \multicolumn{2}{|c|}{ cannot be true because there is also a contradiction. } \\
\hline $\begin{array}{l}\text { Rule 27: Let } \tilde{A} \text { and } \widetilde{B} \text { two simple fuzzy regions, } \\
\text { if Overlap }\left(\tilde{A}_{\max }, \widetilde{B}_{\max }\right) \text { and Covered by } \\
\left(\tilde{A}_{\min }, \quad \widetilde{B}_{\min }\right) \quad \text { then } r \\
\left(\tilde{A}_{\min }, \widetilde{B}_{\max }\right) \in\{\text { Inside, Covered by }\} \text { and } \\
R\left(\tilde{A}_{\max }, \widetilde{B}_{\min }\right) \in\{\text { Covers, } \\
\text { Overlap }\} \text { and vice versa. }\end{array}$ & $\begin{array}{c}\tilde{B}_{\min } \\
\tilde{A}_{\min } \\
\tilde{A}_{\max }\end{array}\left[\begin{array}{cc}C V\left(\tilde{A}_{\min }, \tilde{B}_{\min }\right) & R\left(\tilde{A}_{\min }, \widetilde{B}_{\max }\right) \in\{C V B, O\} \\
R\left(\tilde{A}_{\max }, \tilde{B}_{\min }\right) \in\{C V, C, O\} & \text { Overlap }\left(\tilde{A}_{\max }, \widetilde{B}_{\max }\right)\end{array}\right]$ \\
\hline \multicolumn{2}{|c|}{$\begin{array}{l}\text { Proof: Let } \tilde{A} \text { and } \widetilde{B} \text { two simple fuzzy regions where Overlap }\left(\tilde{A}_{\max }, \widetilde{B}_{\max }\right) \text { and Covers }\left(\tilde{A}_{\min }, \widetilde{B}_{\min }\right)(1) \text {. We } \\
\text { suppose now that } R\left(\tilde{A}_{\min }, \widetilde{B}_{\max }\right) \notin\{\text { Inside, Covered by }\}(2) R\left(\tilde{A}_{\max }, \widetilde{B}_{\min }\right) \notin\{\text { Covers, Contains, Overlap }\} \\
\text { (3). If (2) then } R\left(\widetilde{B}_{\max }, \widetilde{B}_{\min }\right) \notin\{\text { Contains, Covers, Equal }\} \text { or }(1) \text { is false. By considering definition } 1 \text { and } \\
\text { proposition } 2 \text {, (2) cannot be true because there is a contradiction. In the same way, if (3) then } R \\
\left(\widetilde{B}_{\max }, \widetilde{B}_{\min }\right) \notin\{\text { Contains, Covers, Equal }\} \text { or (1) is false. By considering definition } 1 \text { and proposition } 2,(3) \\
\text { cannot be true because there is also a contradiction. }\end{array}$} \\
\hline $\begin{array}{l}\text { Rule 28: Let } \tilde{A} \text { and } \widetilde{B} \text { two simple fuzzy regions, if } \\
\text { Overlap }\left(\tilde{A}_{\max }, \widetilde{B}_{\max }\right) \text { and Covers }\left(\tilde{A}_{\min } \text {, }\right. \\
\left.\tilde{B}_{\min }\right) \text { then } R\left(\tilde{A}_{\min }, \widetilde{B}_{\max }\right) \in\{\text { Inside }\} \text { and } \\
R\left(\tilde{A}_{\max }, \widetilde{B}_{\min }\right) \in\{\text { Covers, } \\
\text { Overlap }\} \text { and vice versa. }\end{array}$ & $\begin{array}{cc}\tilde{B}_{\text {min }} & \tilde{B}_{\max } \\
\tilde{A}_{\min } \\
\tilde{A}_{\max }\end{array}\left[\begin{array}{cc}C V\left(\tilde{A}_{\min }, \tilde{B}_{\min }\right) & R\left(\tilde{A}_{\min }, \widetilde{B}_{\max }\right) \in\{C V B, O\} \\
R\left(\tilde{A}_{\max }, \tilde{B}_{\min }\right) \in\{C V, C, O\} & O\left(\tilde{A}_{\max }, \tilde{B}_{\max }\right)\end{array}\right]$ \\
\hline \multicolumn{2}{|c|}{$\begin{array}{l}\text { Proof: Let } \tilde{A} \text { and } \widetilde{B} \text { two simple fuzzy regions where Overlap }\left(\tilde{A}_{\max }, \widetilde{B}_{\max }\right) \text { and Covers }\left(\tilde{A}_{\min }, \widetilde{B}_{\min }\right)(1) \text {. We } \\
\text { suppose now that } R\left(\tilde{A}_{\min }, \widetilde{B}_{\max }\right) \notin\{\text { Inside, Covered by }\} \text { (2) } R\left(\tilde{A}_{\max }, \widetilde{B}_{\min }\right) \notin\{\text { Covers, Contains, Overlap }\} \\
\text { (3). If (2) then } R\left(\widetilde{B}_{\max }, \widetilde{B}_{\min }\right) \notin\{\text { Contains, Covers, Equal }\} \text { or (1) is false. By considering definition } 1 \text { and (1), } \\
\text { (2) cannot be true because there is a contradiction. In the same way, if (3) then } R\left(\widetilde{B}_{\max }, \widetilde{B}_{\min }\right) \notin\{\text { Contains, } \\
\text { Covers, Equal }\} \text { or (1) is false. By considering definition } 1 \text { and (1), (3) cannot be true because there is also a } \\
\text { contradiction. }\end{array}$} \\
\hline 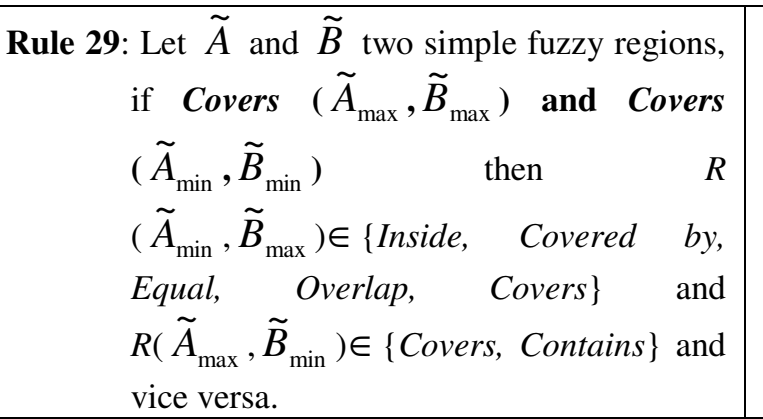 & 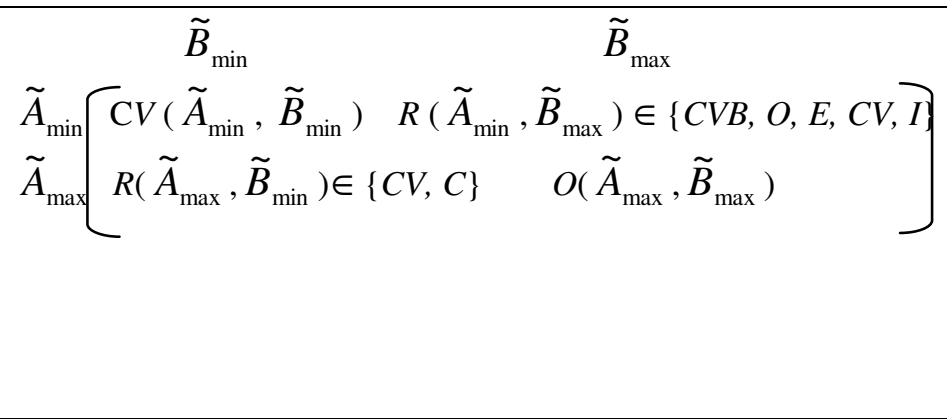 \\
\hline \multicolumn{2}{|c|}{$\begin{array}{l}\text { Proof: Let } \tilde{A} \text { and } \widetilde{B} \text { two simple fuzzy regions where Covers }\left(\tilde{A}_{\max }, \widetilde{B}_{\max }\right) \text { and Covers }\left(\tilde{A}_{\min }, \widetilde{B}_{\min }\right)(1) \text {. We suppose } \\
\text { now that } R\left(\widetilde{A}_{\min }, \widetilde{B}_{\max }\right) \notin\{\text { Covered by, Overlap, Equal, Covers, Inside }\} \text { (2) } R\left(\tilde{A}_{\max }, \widetilde{B}_{\min }\right) \notin\{\text { Covers, } \\
\left.\text { Contains }\} \text { (3). If (2) then } R\left(\widetilde{B}_{\max }, \widetilde{B}_{\min }\right) \notin\{\text { Contains, Covers, Equal }\} \text { or (1) is false. }\right) \text {. By considering } \\
\text { definition } 1 \text { and (1), (2) cannot be true because there is a contradiction. In the same way, if (3) then } R \\
\left.\left(\widetilde{B}_{\max }, \widetilde{B}_{\min }\right) \notin\{\text { Contains, Covers, Equal }\} \text { or (1) is false. }\right) \text {. By considering definition } 1 \text { and (1), (3) cannot be } \\
\text { true because there is also a contradiction. }\end{array}$} \\
\hline
\end{tabular}




\begin{tabular}{|c|c|}
\hline 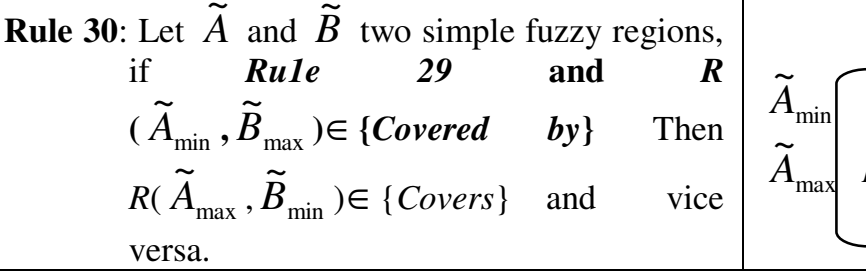 & 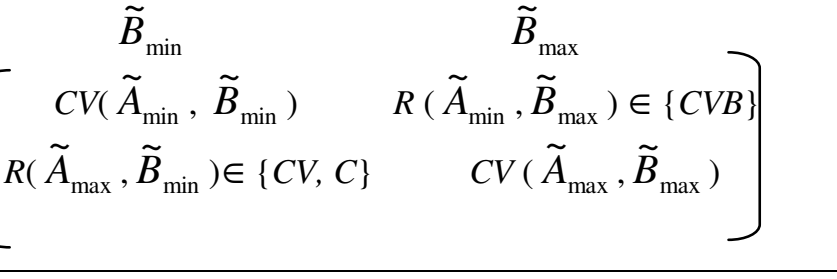 \\
\hline \multicolumn{2}{|c|}{$\begin{array}{l}\text { Oroof: Let } \tilde{A} \text { and } \widetilde{B} \text { two simple fuzzy regions where Rule } 29 \text { and } R\left(\tilde{A}_{\min }, \widetilde{B}_{\max }\right) \in\{\text { Covered by }\} \text { (1). We suppose } \\
\text { now that } R\left(\widetilde{A}_{\max }, \widetilde{B}_{\min }\right) \notin\{\text { Covers }\} \text { (2). By considering definition } 1 \text { and proposition } 2 \text {, if (2) then } R \\
\left(\widetilde{B}_{\max }, \widetilde{B}_{\min }\right) \notin\{\text { Contains, Covers, Equal }\} \text { or (1) is false. Thus, (2) cannot be true because there is a } \\
\text { contradiction. }\end{array}$} \\
\hline $\begin{array}{l}\text { Let } \tilde{A} \text { and } \widetilde{B} \text { two simp } \\
30 \text { and } \boldsymbol{R} \quad\left(\tilde{A}_{\min }, \widetilde{B}\right. \\
R\left(\tilde{A}_{\max }, \widetilde{B}_{\min }\right) \in\{\text { Contai }\end{array}$ & 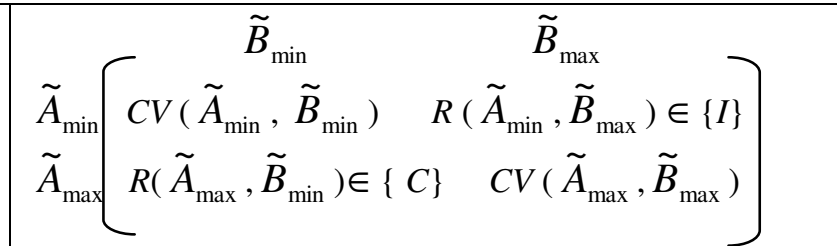 \\
\hline \multicolumn{2}{|c|}{$\begin{array}{l}\text { of: Let } \tilde{A} \text { and } \widetilde{B} \text { two simple fuzzy regions where Rule } 30 \text { and } R\left(\widetilde{A}_{\min }, \widetilde{B}_{\max }\right) \in\{\text { Inside }\} \text { (1). We suppose now } \\
\text { that } R\left(\widetilde{A}_{\max }, \widetilde{B}_{\text {min }}\right) \notin\{\text { Contains }\} \text { (2). If (2) then } R\left(\widetilde{B}_{\max }, \widetilde{B}_{\min }\right) \notin\{\text { Contains, Covers, Equal }\} \text { or (1) is false. } \\
\text { By considering definition } 1 \text { and (1). (2) cannot be true because there is a contradiction. }\end{array}$} \\
\hline
\end{tabular}

\section{References}

AHLQViST, O., KeuKelaA J., and OUKBIR A., 1998, Using Rough classification to Represent Uncertainty in Spatial Data. In Proceedings of the Tenth Annual Colloquium of the Spatial Information Research Centre. P. Firns (editors). 16 - 19 Dec, Dunedin, New Zealand. University of Otago, ISBN 1877139122, pp. 1-10.

AltMAN, D., 1987, Fuzzy set theoretic approaches for handling imprecision in spatial analysis. International Journal of Geographical Information Systems, 8, pp. 271-289.

BÉDARD, Y., 1987, Uncertainties in Land Information Systems Databases, In Proceedings of Eighth International Symposium on Computer-Assisted Cartography, Baltimore, Maryland (USA), 29 Mars - 3 Avril 1987, American Society for Photogrammetry and Remote Sensing and American Congress on Surveying and Mapping, pp. 175-184.

BÉDARd, Y., Rivest, S. and ProulX, M.-J., 2007, Spatial On-Line Analytical Processing (SOLAP): Concepts, Architectures and Solutions from a Geomatics Engineering Perspective. In Data Warehouses and OLAP: Concepts, Architectures and Solutions, Robert Wrembel and Christian Koncilia (Ed.) (London: IRM Press (Idea Group)), Chap. 13, UK, pp. 298-319.

Brown, D.G., 1998, Classification and boundary vagueness in mapping presettlement forest types. International Journal of Geographical Information Systems, 12, 105-129.

BurRough, P.A., 1989, Fuzzy mathematical methods for soil survey and land evaluation. Journal of Soil Science, 40, pp. 477-492.

Burrough, P.A. and Frank, A.U., 1996, Geographic Objects with Indeterminate Boundaries (London: Taylor \& Francis).

Cheng, T., MolenaAR, M. and Lin, H., 2001, Formalizing fuzzy objects from uncertain classification results. International Journal Geographical Information Science, 15(1), pp. $27-42$.

Clementini, E. and Di Felice, P., 1997, Approximate topological relations. International Journal of Approximate Reasoning, 16, pp. 173-204.

CoHN, A.G. and GoTTS N.M., 1996, The 'egg-yolk' representation of regions with indeterminate boundaries. In Proceedings of the GISDATA Specialist Meeting on Spatial 
Objects with Undetermined Boundaries, Burrough, P. \& Frank, A. (Ed.) (Taylor \& Francis), pp. 171-187.

Cohn, A.G., Bennett, B., Gooday J. and GotTs, N.M., 1997, Qualitative Spatial Representation and Reasoning with the Region Connection Calculus, GeoInformatica, 1(3), pp. 275-316.

Chrisman, N.R., 1991, The error component in spatial data. In: Maguire, D.J., Goodchild, M.F and Rhind, D.W. (eds.), Geographical Information Systems: Principles and Applications, Volume 1, pp. 165-174.

Devillers, R., BéDard, Y., Jeansoulin, R., and Moulin, B., 2007, Towards Spatial Data Quality Information Analysis Tools for Experts Assessing the Fitness for Use of Spatial Data, International Journal of Geographical Information Sciences (IJGIS), Vol. 21, 3, pp. 261-282.

DiLO, A., 2006, Representation of and reasoning with vagueness in spatial information: A system for handling vague objects. PhD thesis, ITC, Netherlands, 187p.

Duboisset, M., Pinet, F., KAng M.-Ah. and Schneider M., 2007, A General Framework to Implement Topological Relations on Composite Regions. To appear in: Lecture Notes in Computer Science, Springer.

Duckham, M., Mason, K., Stell, J., and Worboys M., 2001, A formal ontological approach to imperfection in geographic information. Computer, Environment and Urban Systems, 25, pp. 89-103.

EGENHOFER, M.J., 1989, A formal definition of binary topological relations. In Poceedings of the third international conference on Foundations of Data Organisation and Algorithms $(F O D O)$, W.Litin and H.J.Scheck (Ed.) (NY: Springer-Verlag), Lecture notes in computer science, 367, pp. 457-472.

Egenhofer, M. J. and FranzosA, R.D., 1991, Point-set Topological Relations. International journal of geographical Information Systems, 5(2), pp. 161-174.

EgEnhofer, M. and HerRING J., 1990, A mathematical framework for the definition of topological relations. In Proceedings of the Fourth International Symposium on Spatial Data Handling, K. Brassel and H. Kishimoto (Ed.), Zurich, Switzerland, pp. 803-813.

ERwIG, M. and SchNeIDER, M., 1997, Vague regions. In 5th International Symposium on Advances in Spatial Databases (SSD'97), Lecture Notes in Computer Science, 1262, pp. 298-320.

FISHER, P.F., 1999, Models of uncertainty in spatial data. In Geographical Information Systems, P. A. Longley, M. F. Goodchild, D. J. Maguire, and D. W. Rhind (Ed.) (New York: John Wiley \& Sons), pp. 191-205.

FRANK, A.U., 2001, Tiers of ontology and consistency constraints in geographical information systems. In International Journal of Geographical Information Science, 15(7), 667--678.

GodjJevac, J., 1999, Idées nettes sur la logique floue. Presses polytechniques et universitaires romandes, Lausanne.

GoOdCHILD, M.F., 1995, Attribute Accuracy. In Elements of spatial data quality, S.C. Guptill and J.L. Morrison (Ed.) (New York: Elsevier Science inc.), pp. 59-79.

GuPTILL, S.C. and MorRison, J.L., 1995, Spatial data quality. In Elements of spatial data quality, S. C. Guptill and J. L. Morrison (Ed.) (New York: Elsevier Science inc.).

HAZARIKA, S.M. and COHN, A.G., 2001, A taxonomy for spatial vagueness, an alternative egg-yolk interpretation. In Proceeding of the COSIT 2001, The fifth international Conference On Spatial Information Theory, D.R. Montello (Ed.) (California: SpringerVerlag), Lecture Notes in Computer Science, 2205, pp. 92-107.

Hwang, S., and ThILl, J-C., 2005, Modeling Localities with Fuzzy Sets and GIS, In: Cobb M, Petry F, and Robinson V (eds) Fuzzy Modeling with Spatial Information for Geographic Problems, Springer-Verlag, pp. 71-104. 
Mark, D. and Egenhofer, M., 1994, Modeling Spatial Relations Between Lines and Regions: Combining Formal Mathematical Models and Human Subjects Testing, Cartography and Geographical Information Systems, 21 (3), pp. 195-212.

MowreR, H.T., 1999, Accuracy (Re)assurance: Selling Uncertainty Assessment to the Uncertain. In Spatial Accuracy Assessment, Land Information Uncertainty in Natural Ressources, K. Lowell and Jaton A. (Ed.) (Quebec: Ann Arbor Press), pp. 3-10.

PAWLAK, Z., 1994, Rough sets: present state and further prospects. In Third International Workshop on Rough Set and Soft Computing (RSSC '94), pp. 72-76.

Pfoser, D., Tryfona, N. and Jensen, C.S., 2005, Indeterminacy and Spatiotemporal Data: Basic Definitions and Case Study. GeoInformatica, 9(3), pp. 211-236.

RANDELL, D.A. and CoHN A.G., 1989, Modelling topological and metrical properties of physical processes. In Proceedings of the 1st International Conference on Principles of Knowledge Representation and Reasoning (KR'89), R. J. Brachman, H. J. Levesque, and R. Reiter (Ed.) (Morgan Kaufmann), pp. 357-368.

ReIS, R., EgENhOFER, M.J. and MAtos, J., 2006, Topological relations using two models of uncertainty for lines. In. Proceeding of the $7^{\text {th }}$ international Symposium on Spatial Accuracy Assessment in Natural Resources and Environmental Sciences, 5 - 7 July, Lisbon, Portugal, pp. 286-295.

Robinson, V.B. and Thongs, D., 1986, Fuzzy Set Theory Applied to the Mixed Pixel problem of Multi-spectral Land Cover Databases. GIS in Government, 2, B.K. Opitz (Ed.) (Washington D.C: A.Deepak Publication).

RodrigueZ, A., 2005, Inconsistency Issues in Spatial Databases. Lecture Notes In Computer Science, 3300, pp. 237-269.

RoY, A.J. and STELL J.G., 2001, Spatial relations between indeterminate regions. International Journal of Approximate Reasoning, 27, pp. 205-234.

SCHNEIDER, M., 2001, A design of topological predicates for complex crisp and fuzzy regions, In ER '01: Proceedings of the 20th International Conference on Conceptual Modeling, Springer-Verlag, ISBN 3-540-42866-6 pp. 103-116.

Shu H., Spaccapietra, S., Parent, C. and Quesada Sedas, S., 2003, Uncertainty of Geographic Information and its Support in MADS. In Proceeding of the $2^{\text {nd }}$ International Symposium on Spatial Data Quality, Hong Kong.

SMITHSON M., 1989, Ignorance and Uncertainty: Emerging Paradigms (New York: Springer - Verlag).

UbedA, T. and EGENHOFER M., 1997, Topological Error Correcting in GIS. In the Proceedings of International Symposium on Large Spatial Databases, Lecture Notes in Computer Science, Vol. 1262, Springer-Verlag, pp. 283-297.

VAN OORT, P., 2006, Spatial data quality: from description to application. Publication on Geodesy 60 (Netherlands: Geodetic Commission), ISBN 906132295 2, Delft, December.

VARZI, A., 2004, Mereology. In The Stanford Encyclopedia of Philosophy, Edward N. Zalta (Ed.).

WoRbOYs, M.F., 1998, Imprecision in finite resolution spatial data. GeoInformatica, 2, pp. 257-279.

XINMING, T., 2004, Spatial object modeling in fuzzy topological spaces: with applications to land cover change. PhD thesis, University of Twente. ISBN 90-6164-220-5

YAZICI A., ZHU, Q. and SuN N., 2001, Semantic data modeling of spatiotemporal database applications. Int. J. Intell. Syst, pp. 881-904.

Yongming, L. and Sanjiang, L., 2004, A Fuzzy Sets Theoretic Approach to Approximate Spatial Reasoning. IEEE Transaction on Fuzzy Systems, 12(6), pp. 745- 754.

ZADEH, L.A., 1965, Fuzzy sets. Inform. Control, vol. 8, pp. 338-353. 
ZHAN, B.F.,1997, Topological relations between fuzzy regions. In Proceedings of the 1997 ACM Symposium on Applied Computing, (ACM Press), 192-196., ISBN 0-89791-850-9. 
Table 1. Relations' numbers

\begin{tabular}{|l|l|}
\hline The relation between $\left(\tilde{A}_{\max }, \widetilde{B}_{\max }\right)$ & Correspondent matrices \\
\hline Disjoint $\left(\tilde{A}_{\max }, \widetilde{B}_{\max }\right)$ & 1 \\
\hline Contains $\left(\widetilde{A}_{\max }, \widetilde{B}_{\max }\right)$ & $2 \rightarrow 30$ \\
\hline Equal $\left(\widetilde{A}_{\max }, \widetilde{B}_{\max }\right)$ & $31 \rightarrow 52$ \\
\hline Covers $\left(\widetilde{A}_{\max }, \widetilde{B}_{\max }\right)$ & $53 \rightarrow 98$ \\
\hline Covered by $\left(\widetilde{A}_{\max }, \widetilde{B}_{\max }\right)$ & $99 \rightarrow 144$ \\
\hline Inside $\left(\widetilde{A}_{\max }, \widetilde{B}_{\max }\right)$ & $145 \rightarrow 173$ \\
\hline Meet $\left(\widetilde{A}_{\max }, \widetilde{B}_{\max }\right)$ & $174 \rightarrow 177$ \\
\hline Overlap $\left(\widetilde{A}_{\max }, \widetilde{B}_{\max }\right)$ & $178 \rightarrow 242$ \\
\hline
\end{tabular}


Table 2. Clustering results

\begin{tabular}{|c|c|c|}
\hline Cluster's name & $\begin{array}{c}\text { Fuzziness } \\
\text { level }\end{array}$ & Topological relations' numbers (cf. appendix) \\
\hline \multirow[t]{4}{*}{ DISJOINT } & Weakly & $\begin{array}{l}13,14,15,17,41,42,43,44,67,69,70,71,72,74,75,80, \\
113,115,116,117,118,120,121,126,157,159,161,162, \\
193,194,195,196,197,198,199,200,201,204,205,208, \\
213,214,215,216\end{array}$ \\
\hline & Fairly & $\begin{array}{l}16,73,76,119,122,158,175,176,202,203,206,207, \\
209,210,211,212\end{array}$ \\
\hline & Strongly & 174,217 \\
\hline & Completely & 1 \\
\hline \multirow[t]{4}{*}{ CONTAINS } & Weakly & $\begin{array}{l}31,34,36,39,43,44,45,48,51,52,57,59,61,63,67,68, \\
69,71,73,76,77,79,80,82,85,86,88,91,93,94,95,96, \\
102,105,110,113,118,125,128,130,135,137,140,145, \\
146,153,157,163,167,173,181,184,186,189,193,195, \\
198,210,213,218,219,221,223,226,230,232,234,238, \\
240\end{array}$ \\
\hline & Fairly & $\begin{array}{l}8,9,10,11,12,13,14,15,16,17,18,19,20,21,22,23, \\
24,25,26,27,28,29,32,33,37,53,54,55,56,60,103, \\
104,152,178,179,180\end{array}$ \\
\hline & Strongly & $2,3,4,5,7$ \\
\hline & Completely & 6 \\
\hline \multirow[t]{4}{*}{$E Q U A L$} & Weakly & $\begin{array}{l}4,25,26,29,32,34,35,36,37,38,39,40,41,42,43,44, \\
45,46,47,48,49,50,51,52,55,77,78,79,84,91,101, \\
123,124,125,131,138,145,148,170,171,230,231,232, \\
233\end{array}$ \\
\hline & Fairly & $24,30,31,169$ \\
\hline & Strongly & \\
\hline & Completely & \\
\hline \multirow[t]{4}{*}{ COVERS } & Weakly & $\begin{array}{l}7,18,19,21,25,30,35,38,40,41,42,46,47,50,52,53, \\
55,56,57,59,60,61,63,67,68,69,71,73,76,77,79,80, \\
88,93,94,95,96,99,107,108,115,116,123,124,129, \\
132,135,137,139,141,147,156,159,160,166,167,171, \\
183,185,187,192,194,196,201,211,215,218,219,221, \\
223,225,227,228,231,233,235,239,241\end{array}$ \\
\hline & Fairly & $\begin{array}{l}20,49,54,58,62,64,65,66,70,72,74,75,78,82,85,86, \\
89,90,91,92,97,98,136,142,168,220,222\end{array}$ \\
\hline & Strongly & $83,84,87$ \\
\hline & Completely & 81 \\
\hline \multirow[t]{4}{*}{ COVERED BY } & Weakly & $\begin{array}{l}3,12,14,21,22,23,26,30,33,37,39,40,42,44,45,47, \\
49,51,53,61,62,69,70,77,78,83,85,88,90,92,94,99, \\
101,102,103,105,106,107,109,113,114,115,117,122, \\
123,125,126,135,140,141,142,143,151,163,164,166, \\
170,180,186,187,191,195,196,200,212,216,219,220, \\
223,225,226,227,229,232,233,234,235,242\end{array}$ \\
\hline & Fairly & $\begin{array}{l}50,89,95,100,104,108,110,111,112,116,118,119, \\
120,121,124,128,130,132,133,136,137,138,139,144, \\
165,224,228\end{array}$ \\
\hline & Strongly & $129,131,134$ \\
\hline & Completely & 127 \\
\hline
\end{tabular}




\begin{tabular}{|c|c|c|}
\hline \multirow[t]{4}{*}{ INSIDE } & Weakly & $\begin{array}{l}2,9,13,18,22,23,28,29,31,32,36,38,41,43,46,48, \\
51,52,56,59,64,67,72,79,82,88,90,93,98,103,105, \\
107,109,113,114,115,117,122,123,125,126,128,132, \\
133,135,138,140,141,142,143,178,184,185,188,193, \\
194,197,209,214,218,225,226,227,229,230,231,236, \\
240,241\end{array}$ \\
\hline & Fairly & $\begin{array}{l}8,34,35,57,58,99,100,101,102,106,145,152,153, \\
154,155,156,157,158,159,160,161,162,163,164,165, \\
166,167,168,169,170,171,172,173,181,182,183\end{array}$ \\
\hline & Strongly & $146,147,148,149,151$ \\
\hline & Completely & 150 \\
\hline \multirow[t]{4}{*}{ MEET } & Weakly & $\begin{array}{l}9,11,12,15,36,38,39,40,59,61,62,63,64,66,74,80, \\
105,107,108,109,110,112,120,126,153,155,156,161, \\
174,184,185,186,187,188,189,190,191,192,204,205, \\
206,207,213,214,215,216\end{array}$ \\
\hline & Fairly & $10,65,68,111,114,154,175,176,208$ \\
\hline & Strongly & \\
\hline & Completely & 177 \\
\hline \multirow[t]{4}{*}{ OVERLAP } & Weakly & $\begin{array}{l}5,11,17,19,28,33,45,46,47,48,60,63,66,71,75,86, \\
87,92,93,94,98,109,112,117,121,130,133,134,139, \\
140,141,149,155,160,162,164,173,178,180,181,183, \\
184,185,186,187,193,194,195,196,206,207,208,209, \\
210,211,212,213,214,215,216,217,218,219,220,223, \\
225,226,227,228,230,231,232,233\end{array}$ \\
\hline & Fairly & $\begin{array}{l}27,96,97,143,144,172,179,182,188,189,191,192, \\
197,198,200,201,202,203,204,205,221,222,224,229, \\
234,235,240,241\end{array}$ \\
\hline & Strongly & $190,199,236,238,239,242$ \\
\hline & Completely & 237 \\
\hline
\end{tabular}


Table 3. Result of query 3

\begin{tabular}{|l|l|l|}
\hline \multicolumn{1}{|c|}{ Pl.id } & \multicolumn{1}{|c|}{ P2.id } & \multicolumn{1}{c|}{ determine } \\
\hline 11 & 23 & Weakly overlap \\
\hline 45 & 14 & --- \\
\hline 18 & 26 & Strongly Overlap \\
\hline
\end{tabular}




\section{List of figure captions:}

Figure 1. Integration of different spatial representations of a same object (e.g., lake)

Figure 2. A lake with partially fuzzy boundaries

Figure3. Categorization of spatial vagueness

Figure 4. Topological invariants of a simple fuzzy region in (Xinming 2004)

Figure 5. Vague region in (Erwig and Schneider 1997)

Figure 6. Identification of topological relations in (Cohn and Gotts 1996)

Figure 7. Identification of topological relations in (Clementini and Di Felice 1997)

Figure 8. Identification of topological relations in (Xinming 2004)

Figure 9. Minimal and maximal extents for (a) a fuzzy point, (b) a fuzzy line and (c) a fuzzy region

Figure 10. Fuzzy point

Figure 11. Fuzzy lines

Figure 12. Topological invariants according to the line fuzziness

Figure 13. Examples of invalid lines

Figure 14. Region with partial fuzzy boundary

Figure 15. Fuzzy regions

Figure 16. Examples of invalid fuzzy regions

Figure 17. Description of the topological relation between two fuzzy regions: (a) visual content of the matrix, (b) formal identification of the relations between the minimal and maximal extents of the objects involved

Figure 18. Examples of identification of topological relations through a 4-Intersection matrix

Figure 19. Example of a topological relation between a fuzzy region and a crisp region

Figure 20. Controlling the validity of a Disjoint relation

Figure 21. Example of clustering of a topological relation

Figure 22. Evaluation of topological relation's fuzziness

Figure 23. Hierarchical classification of the topological relations

Figure 24. 242 topological relations between fuzzy regions 